\title{
Progress in front propagation research
}

\author{
Joaquim Fort ${ }^{1}$ and Toni Pujol ${ }^{2}$ \\ ${ }^{1}$ Departament de Física, Campus de Montilivi, Universitat de Girona, \\ 17071 Girona, Catalonia, Spain \\ ${ }^{2}$ Departament de Mecànica, Campus de Montilivi, Universitat de \\ Girona, 17071 Girona, Catalonia, Spain
}

\begin{abstract}
We review the progress in the field of front propagation in recent years. We survey many physical, biophysical and crossdisciplinary applications, including reduced-variable models of combustion flames, Reid's paradox of rapid forest range expansions, the European colonization of North America during the XIX century, the Neolithic transition in Europe from 13,000 to 5,000 years before present, the description of subsistence boundaries, the formation of cultural boundaries, the spread of genetic mutations, theory and experiments on virus infections, models of cancer tumors, etc. Recent theoretical advances are unified in a single framework, encompassing very diverse systems such as those with biased random walks, distributed delays, sequential reaction and dispersion, cohabitation models, age structure, systems with several interacting species, etc. Directions for future progress are outlined.
\end{abstract}

PACS numbers: 89.20 -a, 87.23. Ge, 89.65. Ef, 89.75.Fb

\section{INTRODUCTION}

Fronts are observed in many systems with dynamics driven by reaction and diffusion [1,2] (or reproduction and dispersal, in biophysical applications). They describe propagating profiles for the particle concentration, individual number density, temperature, etc. They are widely used in physical models of combustion flames [3], population invasions [4], virus infections [5], tumor growth [6], chemical waves [7], crystallization [8], superconductors [9] and many other interesting phenomena in physical, biophysical, chemical and cross-disciplinary systems $[1,2,10]$.

In the last six years, many new analytical results on front propagation have been published, dealing with sequential reaction and dispersion [11], fronts from biased random walks [12-15], age-structured systems [16], distributed delays [17-22], dispersive variability [22,23], interacting species [24,25], anomalous diffusion fronts [26,27], dispersal kernel effects [28], convective systems [29-31], etc. In this review we present these theoretical advances into a single, unified framework.

We survey many specific physical, biophysical and cross-disciplinary applications of front propagation models. For example, combustion flames is a very active area of research [30-34]. They have been recently described using a single, reduced variable, which makes it possible to derive analytical lower and upper bounds on the propagation speed [32]. We review these results for flame propagation, as well as their extensions to en- compass the effects of mass diffusion, heat convection, and temperature-dependent parameters of heat and mass transport $[33,34]$.

We also discuss the recent explanation of Reid's paradox of rapid forest recolonizations using bimodal kernels with long-distance dispersal in two-dimensional space [11,35].

Physical models are becoming widely applied to human population invasions, including Paleolithic waves of advance in America [36] and Europe [37], as well as to the European colonization of North America in the XIX century [12] and the Neolithic transition in Europe (from 13,000 to 5,000 years before present) $[4,16,20,25]$. Such applications are surveyed here. The description of subsistence boundaries, as well as a recent model on the dynamics leading to the formation of cultural boundaries [38], are also reviewed in this work.

We also review theoretical models and experimental data on the speed of virus infections [5,21,39,40], the spread of genetic mutations [41-43], a recent physical model that describes the spread of cancer tumors by taking into account the lower proliferation rate of migratory cells [44], etc.

Concerning numerical work, in addition to results based on the discretization of differential equations (which are appropriate to some systems, e.g. combustion flames) and fast-Fourier transforms (which require less computing time for integro-difference equations), we also review recent numerical approaches based on reactive random walks on grids (which are more reasonable for biophysical systems in which there is a reproduction process) [11].

Sequential models are an important part of this review. They are necessary in some applications, e.g. to solve Reid's paradox of fast forest recolonizations. The main difference between non-sequential and sequential models is the following. In non-sequential models (Sec. II), reaction and dispersal (of particles or individuals) are simultaneous processes. This is the most well-known approach, and is specially useful in purely physical systems, e.g. in combustion flames (Sec. V). On the other hand, in biophysical applications, the reproduction of individuals replaces the reaction process, and for some biological species (e.g. trees) this is not simultaneous with dispersal. Then, sequential models are more appropriate (Sec. VII). Further refinements are necessary in some cases (e.g., human populations), leading to cohabitation models (Sec. VIII), which are similar mathematically to 
sequential models but apply to different biological systems.

\section{NON-SEQUENTIAL MODELS}

This section surveys non-sequential models of front propagation, for particles (or individuals) performing biased (or anisotropic) random walks. This leads to fronts with speed depending on direction. An interesting application of such anisotropic fronts is the recent explanation (via computer simulations) of the non-homogeneous speed of Neolithic fronts, based on anisotropic diffusion due to enhanced transport along major rivers [15] (Sec. III.B below). Recent theoretical results on anisotropic fronts include a Hamilton-Jacobi derivation of the front position [45], a propagation failure condition for random walks biased in the opposite direction to that of the front propagation [13], velocity-curvature relations [46], nucleation of spiral waves [14], etc. In this section, we will focus our attention on the speed of propagating fronts arising from anisotropic random walks [12].

\section{A. Microscopic derivation from non-isotropic random walks}

In many systems, particles (or individuals) move with a direction-dependent probability, i.e. following a biased (or anisotropic) random walk. Such a behavior is displayed in many phenomena, e.g. particle diffusion in disordered lattices [47], diffusion-limited aggregation [48], experimental populations of micro-organisms [49], human populations invading a geographical region [15], etc.

In order to avoid confusion, it is important to mention that correlation between the directions of successive jumps will not included in this review, because there is no reason to think that such a correlation is relevant in the applications we will tackle. Therefore, we will deal with biased, uncorrelated random walks. In other words, we will allow the probability of jump to depend on the angle relative to a fixed direction. In contrast, in correlated (or persistent) random walks the probability of jump depends on the angle relative to the direction of motion before performing the jump (see, e.g., Sec. 2.2. in [1]).

For the sake of definiteness and clarity, we will deal with a 2-dimensional (2D) space in this section. Extension to $3 \mathrm{D}$ is straightforward, but the $2 \mathrm{D}$ case is relevant to many of the applications that we shall discuss in this review.

Let $p(x, y, t)$ stand for the population (or particle) number per unit area at position $(x, y)$ and time $t$. We define the dispersal kernel $\phi\left(\triangle_{x}, \triangle_{y}, x, y\right)$ as the probability per unit area that an individual (or particle) who was at $\left(x-\triangle_{x}, y-\triangle_{y}, t\right)$ jumps to $(x, y, t+T)[50]$. Let
$T$ stand for the mean time interval between two subsequent jumps (in biophysical applications, usually $T=1$ generation $[4,5])$. Let $R[p(x, y, t)]$ stand for the number of new individuals (or particles) due to the reproduction process (or chemical reactions), produced during the time interval $T$ per unit area centered at $(x, y)$. From these definitions, the evolution equation is usually written as

$$
\begin{aligned}
p(x, y, t+T)-p(x, y, t)= & \int_{-\infty}^{+\infty} \int_{-\infty}^{+\infty} p\left(x-\triangle_{x}, y-\triangle_{y}, t\right) \\
& \phi\left(\triangle_{x}, \triangle_{y}, x, y\right) d \triangle_{x} d \triangle_{y} \\
& -p(x, y, t)+R[p(x, y, t)]
\end{aligned}
$$

where the first and second terms in the right-hand side correspond to the particles (or individuals) arriving minus those leaving an unit area centered at $(x, y)$, and the last one $R[p(x, y, t)]$ is a source term due to chemical reactions (or to the reproduction of individuals).

The so-called diffusion approximation is obtained if second-order Taylor expansions in space and time are performed. Then Eq. (1) becomes

$$
\begin{aligned}
\frac{\partial p}{\partial t}+\frac{T}{2} \frac{\partial^{2} p}{\partial t^{2}}= & -U_{x} \frac{\partial p}{\partial x}-U_{y} \frac{\partial p}{\partial y}+D_{x} \frac{\partial^{2} p}{\partial x^{2}} \\
& +D_{y} \frac{\partial^{2} p}{\partial y^{2}}+D_{x y} \frac{\partial^{2} p}{\partial x \partial y}+F+\frac{T}{2} \frac{\partial F}{\partial t}
\end{aligned}
$$

Here $F$ is the time derivative of $p(x, y, t)$ due to reproduction, i.e. [4]

$$
R[p(x, y, t)]=T F+\frac{T^{2}}{2 !} \frac{\partial F}{\partial t}+\frac{T^{3}}{3 !} \frac{\partial^{2} F}{\partial t^{2}}+\ldots,
$$

$D_{x}, D_{y}$ and $D_{x y}$ are direction-dependent diffusion coefficients,

$$
\begin{gathered}
D_{x}(x, y)=\frac{\left\langle\triangle_{x}^{2}\right\rangle}{2 T}, \\
D_{y}(x, y)=\frac{\left\langle\triangle_{y}^{2}\right\rangle}{2 T}, \\
D_{x y}(x, y)=\frac{\left\langle\triangle_{x} \triangle_{y}\right\rangle}{T},
\end{gathered}
$$

and we have defined

$$
\begin{aligned}
& U_{x}(x, y)=\frac{\left\langle\triangle_{x}\right\rangle}{T}, \\
& U_{y}(x, y)=\frac{\left\langle\triangle_{y}\right\rangle}{T},
\end{aligned}
$$

where the mean value of an arbitrary function of the jump vector $\zeta\left(\triangle_{x}, \triangle_{y}\right)$ is defined as

$$
\left\langle\zeta\left(\triangle_{x}, \triangle_{y}\right)\right\rangle \equiv \int_{-\infty}^{+\infty} \int_{-\infty}^{+\infty} \zeta\left(\triangle_{x}, \triangle_{y}\right) \phi\left(\triangle_{x}, \triangle_{y}, x, y\right) d \triangle_{x} d \triangle_{y}
$$


In general the dispersion kernel $\phi\left(\triangle_{x}, \triangle_{y}, x, y\right)$ can depend on position $(x, y)$ in addition to the jump vector components $\left(\Delta_{x}, \Delta_{y}\right)$. Then, the macroscopic parameters above $\left(D_{x}, D_{y}\right.$, etc.) also depend on position. However, in the homogeneous case we can simply write $\phi\left(\triangle_{x}, \triangle_{y}\right)$ instead of $\phi\left(\triangle_{x}, \triangle_{y}, x, y\right)$.

\section{B. Macroscopic derivation of non-sequential models}

This section gives a simple macroscopic derivation of the same model that has been derived microscopically in the previous section.

Let us assume that there is a maximum possible value for the particle (or individual) number density, $p_{\max }$. In realistic systems, initially all particles (or individuals) are confined into a finite region of space (if this region is very small compared to the dimension of the system, it may be approximated to a point and is called the origin of dispersal). As time goes on, particles (or individuals) will disperse into other regions and react (or reproduce), until the saturation density $p_{\max }$ is locally reached (i.e., $\left.p(x, y, t)=p_{\max }\right)$. The function $p(x, y, t)$ may then be called a front solution, in the sense that it leaves behind a region full of particles or individuals $\left(p(x, y, t)=p_{\max }\right)$, whereas there is an empty region $(p(x, y, t)=0)$ ahead. Let us choose a local $x$-axis along the local propagation direction of the front (for example, for a circular front this direction would be a radial direction from the origin of dispersal). For large enough values of time, we may clearly consider a region centered about this $x$ axis which is sufficiently small so that the $y$-dependence of $p(x, y, t)$ can be neglected. Then Eq. (2) becomes simply

$$
\frac{\partial p}{\partial t}+\frac{T}{2} \frac{\partial^{2} p}{\partial t^{2}}=-U_{x} \frac{\partial p}{\partial x}+D_{x} \frac{\partial^{2} p}{\partial x^{2}}+F+\frac{T}{2} \frac{\partial F}{\partial t} .
$$

It is easy to see that this equation can be also derived by combining the following set of phenomenological equations

$$
\left\{\begin{array}{l}
\frac{\partial p}{\partial t}+\frac{\partial J}{\partial x}=F \\
J+\tau \frac{\partial J}{\partial t}=U_{x} p-D_{x} \frac{\partial p}{\partial x}
\end{array}\right.
$$

where $J$ is the diffusion flux and $\tau \equiv \frac{T}{2}$ is called the relaxation time. The first equation of this set is just a mass balance equation, whereas the second one is a firstorder Taylor expansion for a time-delayed flux,

$$
J(x, t+\tau)=U_{x} p-D_{x} \frac{\partial p}{\partial x} .
$$

From this equation, we can say that the macroscopic effect arising from a direction-dependent microscopic motion of the particles is to introduce an additional flux $U_{x} p$ to the usual diffusion flux $-D_{x} \frac{\partial p}{\partial x}$. In contrast, the effect of a finite jump time $(\tau \neq 0)$ is to introduce a delay in the whole flux $J$. Equation (11) for the non-delayed limit $\tau=0$ is well-known to arise from biased random walks [51].

This simple macroscopic derivation of Eq. (9) from the set (10) is appealing because of its simplicity. However, it is not enough in general to apply the model to experimental data. The reason is that the set (10) is written in terms of the macroscopic parameters $\tau, U_{x}$ and $D_{x}$. But in specific applications, the identification of the relaxation time $\tau$ as half de time interval between successive jumps (i.e. $\tau=T / 2$ ), the macroscopic 'speed' as $U_{x}$ as Eq. (7), and the $x$-diffusion coefficient $D_{x}$ as Eq. (4) are usually necessary in order to estimate the numerical values of $\tau, U_{x}$ and $D_{x}$ (Sec. III). And these three key results can be derived only from microscopic models (e.g., that in the previous subsection) but not from macroscopic ones such as the set (10).

The same model considered in this subsection and the previous one has been recently derived also for the case in which natality and mortality rates may depend on age (Appendix A).

\section{The speed of non-sequential fronts}

A reproduction function that has been widely applied in biophysics problems is the logistic function

$$
F=r_{L} p(x, y, t)\left(1-\frac{p(x, y, t)}{p_{\max }}\right),
$$

where $r_{L}$ is called the initial growth rate and $p_{\max }$ the saturation density.

There are several ways to introduce Eq. (12). Usually, experimental data for many biological populations $[52,53]$ are considered as a valid justification for its use. Interestingly, Hall [53] has argued that the high-density limit (corresponding to the last term) of Eq. (12) has been repeatedly compared favorably to experimental data for populations in the laboratory (specially of microorganisms) but not for wild populations (however, this does not change the front speed, which is our main aim in this section).

Another way to introduce Eq. (12) is by means of agestructured derivations, e.g. that presented in Appendix A (this is more complicated mathematically, but it clearly shows that Eq. (12) takes into account the net effect of births and deaths of individuals).

As explained in Sec. II.A, up to second order the finitedifference Eq. (1) becomes the partial differential Eq. (2) and $T$ (i.e., the rest time between two successive jumps of particles or migrations of individuals) plays the role of a diffusive delay time. As we shall now see, this secondorder approximation makes it possible to derive an analytical result for the front speed.

In this review we will not discuss the problem of the dependence of the front speed on direction $[46,14,54]$. Instead, in order to deal with the applications we are interested in, it will be simpler to focus our attention into 
the speed of fronts along the $x$ direction. The front speed can be found most easily by assuming that for $t \rightarrow \infty$ the front curvature is negligible at scales much larger than that of individual dispersal events [55]. In other words, we consider a region centered about the $x$ axis which is sufficiently small so that the $y$-dependence of $p(x, y, t)$ can be neglected. Then Eq. (2) becomes simply Eq. (9), i.e.

$$
\frac{\partial p}{\partial t}+\frac{T}{2} \frac{\partial^{2} p}{\partial t^{2}}=-U_{x} \frac{\partial p}{\partial x}+D_{x} \frac{\partial^{2} p}{\partial x^{2}}+F+\frac{T}{2} \frac{\partial F}{\partial t} .
$$

Let $c$ stand for the front speed. We look for constantshape solutions with the form

$$
p=p_{0} \exp [-\lambda(x-c t)]
$$

as $x-c t \rightarrow \infty$, with $c>0$ and $\lambda>0$. In this way, from Eqs. (13) and (12) for $p \simeq 0$ (or $x-c t \rightarrow \infty$ ) up to first order, we obtain the characteristic equation

$$
\lambda^{2}\left(D_{x}-\frac{T c^{2}}{2}\right)-\lambda\left(c-U_{x}-\frac{r_{L} T c}{2}\right)+a=0 .
$$

Solving this equation for $\lambda$ and requiring for it to be real, we obtain the condition

$$
\begin{aligned}
f(c) & \equiv c^{2}\left(1+\frac{r_{L} T}{2}\right)-2 c U_{x}\left(1-\frac{r_{L} T}{2}\right) \\
& -4 r_{L} D_{x}-U_{x}^{2} \geq 0 .
\end{aligned}
$$

It is easily seen that $f(c)$ is convex from below, and that the equation $f(c)=0$ has one negative and one positive root for $c$, say $c_{-}$and $c_{+}$. Therefore, the minimum possible value for $c>0$ corresponds to $c_{+}$. Let us now assume, as usual, that this minimum possible speed $c_{+}$is that selected by the front (this is usually called linear or marginal stability analysis $[1,2])$. In this way we finally obtain

$$
c=\frac{U_{x}\left(1-\frac{r_{L} T}{2}\right)+2 \sqrt{a D_{x}\left(1+\frac{r_{L} T}{2}\right)^{2}-\frac{r_{L} T}{2} U_{x}^{2}}}{\left(1+\frac{r_{L} T}{2}\right)^{2}} .
$$

Below we consider some limiting cases.

\section{Non-biased, delayed fronts}

The non-biased case corresponds to an isotropic kernel, so $\left\langle\triangle_{x}\right\rangle=0$ and $U_{x}=0$ from Eq. (7). In this limit, we recover from Eq. (17) a result that has been referred to as hyperbolic reaction-diffusion (HRD) [1], namely

$$
\lim _{U_{x} \rightarrow 0} \stackrel{c}{\rightarrow}=\frac{2 \sqrt{r_{L} D}}{1+r_{L} T / 2}
$$

where, according to Eqs. (4)-(5) for isotropic kernels,

$$
D \equiv D_{x}=D_{y}=\frac{\left\langle\triangle^{2}\right\rangle}{4 T}
$$

For later use, we note that the corresponding (HRD) evolution equation is Eq. (13) in the non-biased limit $\left(U_{x} \rightarrow 0\right)$,

$$
\frac{\partial p}{\partial t}+\frac{T}{2} \frac{\partial^{2} p}{\partial t^{2}}=D \frac{\partial^{2} p}{\partial x^{2}}+F+\frac{T}{2} \frac{\partial F}{\partial t},
$$

with $F$ given by the logistic reproduction rate (12).

\section{Biased, non-delayed fronts}

For a biased random walk with negligible delay time $\left(T \ll \frac{1}{r_{L}}\right)$, Eqs. (17) and (13) become

$$
\begin{gathered}
c=U_{x}+2 \sqrt{r_{L} D_{x}} \\
\frac{\partial p}{\partial t}=-U_{x} \frac{\partial p}{\partial x}+D_{x} \frac{\partial^{2} p}{\partial x^{2}}+F
\end{gathered}
$$

where $F$ is again given by Eq. (12).

\section{Non-biased, non-delayed fronts}

In the latter two equations, if we consider the additional limit of a non-biased random walk $\left(U_{x} \rightarrow 0\right)$, we come to Fisher's speed

$$
c \rightarrow 2 \sqrt{r_{L} D}
$$

and

$$
\frac{\partial p}{\partial t}=D \frac{\partial^{2} p}{\partial x^{2}}+F
$$

which is Fisher's well-known reaction-diffusion equation [56], with $F$ given by the logistic reproduction rate (12).

\section{Connection between microscopic and macroscopic dispersal parameters}

In order to apply Eq. (17), we need to assume some function for the kernel $\phi\left(\triangle_{x}, \triangle_{y}\right)$ appearing in Eqs. (4) and (7). As explained in Sec. II.A, we consider uncorrelated random walks by assuming that the length and direction of jumps are independent, i.e.

$$
\phi\left(\triangle_{x}, \triangle_{y}\right)=\Psi(\triangle) \Phi(\theta)
$$

where $\triangle=\sqrt{\triangle_{x}^{2}+\triangle_{y}^{2}}$ and $\theta=\tan ^{-1} \frac{\triangle_{y}}{\triangle_{x}}$. Correspondingly, we write the normalization condition of the kernel, namely

$$
\int_{-\infty}^{+\infty} \int_{-\infty}^{+\infty} \phi\left(\triangle_{x}, \triangle_{y}\right) d \triangle_{x} d \triangle_{y}=1
$$


as a normalization condition for the length jump probability distribution,

$$
\int_{0}^{\infty} \Psi(\triangle) \Delta d \triangle=1
$$

and another one for the probability distribution of the jump direction,

$$
\int_{0}^{2 \pi} \Phi(\theta) d \theta=1
$$

Several functions $\Phi(\theta)$ have been used in the literature on biased random walks $[49,57]$. For the purposes of the present review, it will be interesting to consider the simple form [12]

$$
\Phi(\theta)=a \pm b \cos \theta
$$

where $b \geq 0$ and $a=\frac{1}{2 \pi}$ from the normalization condition (28). Therefore

$$
\Phi(\theta)=\frac{1}{2 \pi} \pm b \cos \theta .
$$

The following two cases can be considered.

(i) The positive sign in Eq. (30) corresponds to the case in which the random walk is biased towards the local front propagation direction (recall that in the previous subsection, we have computed macroscopic front speeds along this $x$-direction $(\theta=0))$. Then, the jump probability along the front direction $(\theta=0)$ is $\Phi=a+b$. It decreases with increasing values of $|\theta|$, down to the minimum $\Phi=a-b$ (which is attained for $\theta=\pi$ ).

(ii) The negative sign in Eq. (30) corresponds to the case in which the minimum jump probability is attained along the local front propagation direction, namely $\Phi(\theta=$ $0)=a-b$. It increases for increasing values of $|\theta|$, up to the maximum possible value $\Phi(\theta=\pi)=a+b$. Note that the kernel (30) is a probability distribution, so it must be positive for all values of $\theta$. Thus, in case (ii) we have the condition

$$
0 \leq b \leq \frac{1}{2 \pi}
$$

In both cases (i) and (ii), the dimensionless parameter

$$
\beta \equiv \frac{b}{a}=2 \pi b \geq 0
$$

may be called the bias of the random walk. In case (ii), we see from Eq. (31) that

$$
0 \leq \beta \leq 1
$$

Note that we may have case (i) at one point of space and case (ii) in another point because $U_{x}$, as defined by Eq. (7), is space-dependent in general. This may be interesting to describe systems with non-homogeneous rates of front spread. For example, in biological invasions individuals may have a preference to jump in the local front direction at some areas (case (i), $U_{x}>0$ ), e.g. because they are attracted by more favorable habitats. But if other regions are difficult to colonize, the random walk of individuals may be strongly biased against the local front invasion direction (case (ii), $U_{x}<0$ ) and the front speed will become slower. An application of case (ii) $\left(U_{x}<0\right)$ is presented in the next Sec. III.A.

The diffusion coefficient $D_{x}$ and the macroscopic bias parameter $U_{x}$ appearing in the front speed, Eq. (17), can finally be related to the microscopic bias parameter $b$, by using Eqs. (25) and (30) to perform the integrations in Eqs. (4) and (7). This yields

$$
\begin{gathered}
D_{x}=\frac{\left\langle\Delta_{x}^{2}\right\rangle}{2 T}=\frac{\left\langle\Delta^{2}\right\rangle}{4 T}=\frac{1}{4 T} \int_{0}^{\infty} \Psi(\triangle) \Delta^{3} d \triangle \\
U_{x}=\frac{\left\langle\triangle_{x}\right\rangle}{2 T}= \pm \pi b \frac{\langle\Delta\rangle}{T}= \pm \frac{\pi b}{T} \int_{0}^{\infty} \Psi(\triangle) \Delta^{2} d \triangle .
\end{gathered}
$$

\section{APPLICATIONS OF NON-SEQUENTIAL MODELS}

\section{A. European invasion of North America during the XIX century}

As an illustration, in this subsection we consider the human population front colonizing North America in the period 1790-1910 [12]. The front speed can be easily determined, either from detailed population maps [58] or from the center-of-mass population trajectory [59]. Both approaches yield essentially the same range for the observed speed, namely $(13.5 \pm 0.8) \mathrm{km} / \mathrm{yr}(95 \%$ confidence-level interval) [60]. On the other hand, mean migration data of individuals are strongly biased in the direction opposite to that of the front propagation [61]. Therefore, we are dealing with case (ii) discussed at the end of the former section.

The parameter values for this application can be estimated as follows. Lotka fitted a logistic growth function (12) to the population of the United States and obtained for the initial growth rate $r_{L}=0.031 \mathrm{yr}^{-1}$ [52]. It is worth to note that this estimation agrees almost exactly with independent estimations for human populations in other places and time intervals [4]. Diffusion parameters are more difficult to estimate. Sometimes a relatively small sample of migration distances from genealogies are combined with persistence data from other sources [60], but demographers have pointed out that genealogy data are not representative of the whole population [61]. Ferrie has analyzed migration distances for the United States in the XIX century [61]. Using his data for regions with more than 500 observations (i.e., a total of 3,804 individuals) yields $D_{x}=6075 \mathrm{~km}^{2} /$ yr using Eq. (34). Finally, 
we can estimate the macroscopic bias parameter $U_{x}$ using Eq. (35) and values for $\frac{\langle\Delta\rangle}{T}=24.42 \mathrm{~km} / \mathrm{yr}[62]$ and $b$ (or, equivalently, $\beta$ ),

$$
U_{x}=-\frac{\beta}{2} \frac{\langle\Delta\rangle}{T}
$$

The value of $\beta$ can be also estimated from Ferrie's data cited above, but we prefer to use the anisotropy parameter $\beta$ as a free parameter (horizontal axes in Fig. 1) because Ferrie's data contain only a few directions, so it does not seem possible to obtain a precise value for $\beta$ [63].

In Fig. 1 we present the speed predicted by Eq. (17) as a function of the random walk bias $\beta$ [see Eq. (33)]. The HRD speed (18), which corresponds to the non-biased limit $(\beta=0)$, is also shown. It is seen that the difference of the biased model relative to the HRD speed can be substantial, as large as 30\%. We also include Fisher's speed, Eq. (23). In Fig. 1, the biased model is seen to be compatible with the observed speed for high enough values of $\beta$. We would like to stress, however, that it is difficult to estimate the bias parameter $\beta$ precisely with the data available [12]. At this stage the important conclusions are: (i) A bias in the random walk can have a very important effect on the front speed (Fig. 1); (ii) The biased model leading to Eq. (17) is free of some relatively strong assumptions (concerning e.g. the fractal nature of pathways) that are used in alternative physical models of population invasions $[60,36]$.

Note that in the biased model, there is an advection term and a diffusion term (first and second terms in the RHS of Eq. (13), respectively). Their relative importance as a function of distance can be estimated by means of the Peclet number,

$$
\mathrm{Pe}=\frac{U_{x} L}{D_{x}},
$$

which for the human invasion application in this section becomes of order 1 for distances $L$ of the order of $500 \mathrm{~km}$. This is a distance scale to similar to that in which the front speed is measured $[58,59]$, so this illustrative application supports our proposal that both advection and diffusion can be important in biophysical applications of front propagation models.

A more elaborated (cohabitation) model will be discussed in Sec. VIII.C.

\section{B. Non-homogeneous dispersion kernels and non-isotropic fronts}

Davison, Dolukhanov, Sarson and Shukurov [15] considered the two-dimensional Eq. (2) with $D_{x}=D_{y} \equiv D$ and $D_{x y}=0$ in the non-delayed limit $(T \rightarrow 0)$,

$$
\frac{\partial p}{\partial t}=-(\vec{U} \cdot \vec{\nabla}) p+\vec{\nabla}(D \vec{\nabla} p)+F
$$

where $F$ is given by the logistic reproduction rate (12). In their model, the advective speed $\vec{U}$, diffusion coefficient $D$ and carrying capacity $p_{\max }$ are position-dependent. The motivation for this approach was the Neolithic transition in Europe, i.e. the invasion of Europe by farming populations from the Near East in the period 13,000 to 5,000 years Before Present. Archaeological data imply that the front of Neolithic humans (farming populations) had an average speed of about $1 \mathrm{~km} / \mathrm{yr}$. However, there are significant regional variations, e.g. a retardation of the spread to the Alps and to latitudes above $54^{\circ} \mathrm{N}$, and increased propagation speeds along the Danube and Rhine valleys and along the Mediterranean coast $[64,65]$. In order to model such regional variations, the term with the advective speed $\vec{U}$ was included in Eq. (38) and the parameter values were assumed non-uniform. The term with advective speed $\vec{U}$ accounts for the enhanced motivation of the population to move in some particular directions (e.g., along river valleys). As shown in Sec. II above, this term arises naturally if the dispersion kernel is non-isotropic (see, e.g., Eqs. (2) and (7)(8)). So this model takes into account the effects of (i) non-isotropic dispersion and (ii) non-homogeneous parameter values. Motivated by anthropological observations, the authors of Ref. [15] assumed that the initial growth rate is uniform. They used the value $r_{L}=0.02$ $\mathrm{yr}^{-1}$. The advection speed $\vec{U}$ was assumed tangent to the Danube and Rhine rivers, pointing to the direction of locally decreasing population density, and restricted to a strip of $20 \mathrm{~km}$ width around the river (assuming a Gaussian shape), with a maximum value of $|\vec{U}|=5$ $\mathrm{km} / \mathrm{yr}$ (this value is motivated by the spread rate of the Linear Pottery (LBK) culture along the Danube-Rhine corridor [64]). Similarly, sea travel was included by assuming $\vec{U}$ tangent to the coast, with a maximum value of $|\vec{U}|=10 \mathrm{~km} / \mathrm{yr}$ (motivated by the spread rate along the Mediterranean coast [65]). Since early farming was not possible at altitudes higher than $1000 \mathrm{~m}$, both $p_{\max }$ and $D$ were assumed to decrease to zero smoothly from $900 \mathrm{~m}$ to $1000 \mathrm{~m}$ height. The effect of the harsh climate in the north was modelled by assuming linear functions of $p_{\max },|\vec{U}|$ and $D$ with latitude (in such a way that these parameters where reduced by a factor approximately $\frac{1}{2}$ from Greece to Denmark). The background diffusivity assumed in Ref. [15] was $D=12.5 \mathrm{~km}^{2} / \mathrm{yr}$ and, to allow for sea travel (which is implied by the archaeological data), $D$ was assumed to reduce exponentially into the seas with distance from the shore, over a length scale of $10 \mathrm{~km}$. By numerically integrating Eq. (38) on a spherical surface (with larger horizontal mesh sizes for lower latitudes), this non-homogeneous model was shown to predict an accelerated spread via the Rhine-Danube valleys (Fig. 4 in Ref. [15]), in agreement with the archaeological data. Importantly, this key result was not obtained if the term with the advective speed $\vec{U}$ in Eq. 
(38) was neglected, even for non-homogeneous parameter values (Fig. 3 in Ref. [15]). Therefore, the role of advective speed in reaction-diffusion equations (due to nonisotropic dispersal) seems to be of utmost importance in this application. More recently, this model has been also applied to a two-source description of the Neolithic transition (including non-farming sites with ceramics in North-Eastern Europe) with one source in the Near East and a second source in the Urals [66].

\section{Subsistence boundaries}

Cohen [67] proposed a model for a single population, e.g. Neolithic humans (i.e., farmers invading an area originally populated by Paleolithics, i.e. hunter-gatherers). In this model birth and death rates, $b(\sigma)$ and $d(\sigma)$, depend on land fertility $\sigma$, and thus on position. After making some assumptions on the analytical forms of the functions $b(\sigma)$ and $d(\sigma)$, Cohen's final expression for the contribution of the birth and death rates on the rate of change of the population density has the form

$$
\left.\frac{\partial n}{\partial t}\right|_{b, d}=\frac{1}{\tau(n)} n(1-n),
$$

where $n \equiv p / p_{\max }$, and the saturation density $p_{\max }$ also depends on the land fertility $\sigma$, and thus on position. Cohen also noted that, for such a position-dependent saturation density $p_{\max }$, the usual Fickian diffusion flux $\vec{J}_{D}=-D \vec{\nabla} p$ would not vanish even at points where the population density has already reached it maximum possible value $\left(p=p_{\max }\right)$. In order to avoid this, Cohen suggested to add a new flux $\vec{J}_{F}$ to $\vec{J}_{D}$ such that (i) $\vec{J}_{F}$ is proportional to the population density $p(x, y, t)$; and (ii) there is no total flux in points and times where $p$ reaches its saturation value $p_{\max }$. This yields $\vec{J}_{F}=D p\left(\vec{\nabla} p_{\max }\right) / p_{\max }$, so the total flux is

$$
\vec{J}=\vec{J}_{D}+\vec{J}_{F}=-D p_{\max } \vec{\nabla}\left(p / p_{\max }\right)
$$

and its contribution to the rate of change of the population density is

$$
\left.\frac{\partial n}{\partial t}\right|_{\vec{J}}=-\frac{\vec{\nabla} \cdot \vec{J}}{p_{\max }}=\frac{\vec{\nabla} \cdot\left(D p_{\max } \vec{\nabla} n\right)}{p_{\max }} .
$$

If in some region the land fertility is so low that farmers cannot survive (e.g., a mountain), then $p_{\max }=0$ and Eq. (40) yields $\vec{J}=0$. Therefore, there is no flux across such a 'subsistence boundary' and no Neolithic population beyond it. Cohen suggests that this offers a possible explanation for the persistence of isolated languages in mountainous regions of Europe (e.g. the Basque, the languages of the Caucasus and those of the Urals), in the form of such subsistence boundaries around some mountainous regions, across which no Neolithic population advance would have occurred. In such cases, the Paleolithics would have had time enough to adopt farming (instead of being overwhelmed by a much more numerous Neolithic population density, as it presumably happened in most of Europe). An open problem is to find a microscopic, non-heuristic derivation for the additional flux $\vec{J}_{F}$. This model is an interesting proposal, and its implications on the description of subsistence boundaries deserve further development.

Cohen's final evolution equation is obtained by adding up Eqs. (39) and (41),

$$
\frac{\partial n}{\partial t}=\frac{1}{\tau(n)} n(1-n)+\frac{\vec{\nabla} \cdot\left(D p_{\max } \vec{\nabla} n\right)}{p_{\max }} .
$$

This equation is an alternative to Fisher's classical equation (24) in non-homogeneous spaces (for the homogeneous case, Cohen's equation becomes equivalent to Fisher's equation in practice, because the dependence $\tau(n)$ is weak [38]). Cohen's equation (42) will be generalized to the important case of several interacting populations in the next subsection.

\section{Cultural boundaries}

Recently, Ackland, Signitzer, Stratford and Cohen [38] have generalized Cohen's model to deal with several interacting populations. Their model displays 'cultural boundaries' after which a population trait (e.g., language, ceramics, etc.) does not extend because the advantageous trait (farming) is thereafter no longer carried along by the invading population (Neolithics) but by an indigenous one that has adopted it (Paleolithic communities who have become farmers, instead of hunter-gatherers as in the past). There is a crucial difference between cultural and subsistence boundaries. Whereas subsistence boundaries (summarized in the previous subsection) can form only around areas with too low land fertility to sustain agriculture (e.g., mountainous regions), cultural boundaries can form even in homogeneous land. Thus, here we will consider the case of uniform parameter values, which is mathematically simpler. For simplicity, Ackland et al. [38] consider equations of the form (42) with the same diffusion coefficient for all populations. Following again Cohen's model [67] (see the previous subsection), they obtain that the generalization of Eq. (42) to three interacting populations is, for the case of uniform parameters,

$$
\left\{\begin{aligned}
\frac{\partial n_{F}}{\partial t}= & \frac{1}{\tau_{F}} n_{F}\left(1-n_{F}-n_{F H}-n_{F X}\right) \\
& +D \frac{\partial^{2} n_{F}}{\partial r^{2}}+\lambda n_{F} n_{X}\left(n_{F}-n_{X}\right), \\
\frac{\partial n_{H}}{\partial t}= & \frac{1}{\tau_{H}} n_{H}\left(1-n_{H}-n_{H F}-n_{H X}\right) \\
& +D \frac{\partial^{2} n_{H}}{\partial r^{2}}-\gamma n_{H}\left(n_{F}+n_{X}\right), \\
\frac{\partial n_{X}}{\partial t}= & \frac{1}{\tau_{X}} n_{X}\left(1-n_{X}-n_{X F}-n_{X H}\right) \\
& +D \frac{\partial^{2} n_{X}}{\partial r^{2}}+\gamma n_{H}\left(n_{F}+n_{X}\right)-\lambda n_{F} n_{X}\left(n_{F}-n_{X}\right),
\end{aligned}\right.
$$


where $n_{i} \equiv p_{i} / p_{\max } i$ and $n_{i j} \equiv n_{j} p_{\max } j / p_{\max i}$ (with $i, j=F, H, X), p_{F}$ is the population density of the invading Neolithic farmers, $p_{H}$ that of the invaded Paleolithic hunter-gatherers, and $p_{X}$ that of acculturated huntergatherers (i.e., individuals who were hunters but have adopted farming and their descendants, so that they may retain the Paleolithic language, cultural styles, etc.). The interaction terms describe learning of farming by hunters $H$ (who thus become converts $X$ ) with strength $\gamma$, and competition between $F$ and $X$ farmers (e.g., for a cultural style or language) with strength $\lambda$ (assumed proportional to the frequency of encounters and the disparity in population size).

In general, the parameters $\tau_{i}$ depend on the total population density according to Cohen's model (previous subsection), but this dependence was found to be weak in Ref. [38], so it is not included here for simplicity.

In Fig. 2 we present typical numerical integrations of the model (43). It is seen that, even for uniform land fertility, a halo of converts $X$ forms (due to the learning process, i.e. the term with $\gamma$ ). This halo gradually grows until the convert population $(X)$ reaches saturation, and the Neolithic farmers $(F)$ thereafter become extinguished (due to the competition process, i.e. the term with $\lambda$, that changes sign after $n_{X}$ becomes larger than $n_{F}$ ). The role of the $F$-population is played by the $X$-population after some point $\left(t \simeq t_{8}=2,000 \mathrm{yr}\right.$ or $r \simeq 2,400 \mathrm{~km}$ in Fig. 2). There, a cultural boundary forms because farming is from that point on propagated by converts $X$ instead of Neolithics $F$ (right of Fig. $2)$. After the cultural boundary, traits other than farming (e.g., a cultural style, language, etc.) are Paleolithic and no longer Neolithic (as they were before the cultural boundary had been reached, i.e. to the left of Fig. 2).

According to Ackland et al. [38], the parameter $\gamma$ approximately sets the timescale for the formation of the cultural boundary as $1 / \gamma$. This is confirmed for the parameter values used in Fig. 2, where $1 / \gamma=2,000 \mathrm{yr}$. The speed of the $F$-population front in Fig. 2 is $c \simeq 1.2$ $\mathrm{km} / \mathrm{yr}$ (no analytical equation for it has been yet derived for the three-population model (43)). The approximate location of the boundary can thus be predicted as this speed times $1 / \gamma(r \simeq c / \gamma \simeq 2,400 \mathrm{~km}$ in Fig. 2$)$.

For $\lambda=0$, the model due to Aoki et al. [68] is recovered and the cultural boundary does not form. Instead, coexistence of the $F$ and $X$ populations continues throughout, and the genetic cline can be computed as $n_{F} /\left(n_{F}+n_{H}+n_{X}\right)[68]$.

For non-uniform geographies (space-dependent parameter values), the cultural boundary will of course form sooner or later than for the case of uniform parameter values (Fig. 2), but the final state is the same.

Ackland and co-workers suggest that their model may explain important phenomena at a continental scale, such as the boundary of the LinearBank Keramik (LBK) style in Europe, the present-day distribution of Dravidian speakers in India, or that of Bantu speakers in Africa.

Further progress on this line of research could include a careful evaluation of the parameter values from independent observations of interacting population dynamics, so that the distance where the cultural boundary forms (relative to the origin of dispersal) could be predicted and compared to the observed values. Also, it would be very important to justify (using independent observations) the form that the competition term $\lambda n_{F} n_{X}\left(n_{F}-n_{X}\right)$, specially the assumption that it is proportional to the disparity of population sizes $\left(n_{F}-n_{X}\right)$. Indeed, in this model the prediction of cultural boundaries seems to require that the sign of this term changes as the $X$ population becomes large enough (leading to the extinction of the $F$ population) .

\section{E. The spread of genetic mutations}

Some authors have considered a generalization of Fisher's equation (24) for several species or populations $i=$ $1,2, \ldots, n$ given by the evolution equations [43]

$$
\frac{\partial p_{i}}{\partial t}=\left(\rho_{i}^{+}-\rho_{i}^{-}\right) p_{i}+D_{i} \nabla^{2} p_{i}+\sum_{j \neq i}\left[k_{j i} p_{j}-k_{i j} p_{i}\right]
$$

where species $i$ has number density $p_{i}$, replication and disappearance rates $\rho_{i}^{+}$and $\rho_{i}^{-}$, respectively (generally dependent on the composition vector $\vec{p}=$ $\left.\left(p_{1}, p_{2}, \ldots, p_{n}\right)\right)$, and diffusion coefficient $D_{i}$, whereas $k_{i j}$ is the rate of transformation of species $i$ into $j$.

Vlad, Cavalli-Sforza and Ross have analyzed the evolution of the fractions of the different species or populations, $\gamma_{i} \equiv p_{i} / p$. For example, in chemistry $\gamma_{i}$ are molar fractions, whereas in population genetics they are gene frequencies. After lengthy mathematical transformations, Eq. (44) leads to the following, very interesting evolution equations for the total population density $p=\sum p_{i}$ and for the fractions $\gamma_{i}[43]$

$$
\begin{gathered}
\frac{\partial p}{\partial t}=\left(\sum \gamma_{i} \rho_{i}^{+}-\sum \gamma_{i} \rho_{i}^{-}\right) p+\nabla^{2}\left(p \sum \gamma_{i} D_{i}\right) \\
+\sum_{j \neq i}\left[k_{j i} p_{j}-k_{i j} p_{i}\right], \\
\frac{\partial \gamma_{i}}{\partial t}+\vec{\nabla} \cdot\left(\overrightarrow{v_{i}} \gamma_{i}\right)=R_{i}+D_{i} \nabla^{2} \gamma_{i}+\gamma_{i} \vec{\nabla} \cdot \overrightarrow{v_{i}} \\
+\sum_{j \neq i}\left[k_{j i} p_{j}-k_{i j} p_{i}\right],
\end{gathered}
$$

where

$$
\begin{gathered}
\overrightarrow{v_{i}}=-2 D_{i} \vec{\nabla} \ln p \\
R_{i}=\gamma_{i}\left[\delta \rho_{i}^{+}-\delta \rho_{i}^{-}\right] \\
-\gamma_{i}\left\{\sum \delta D_{i}\left[\nabla^{2} \gamma_{i}+2(\vec{\nabla} \ln p) \cdot \vec{\nabla} \gamma_{i}\right]\right\} \\
+\delta D_{i} \gamma_{i} \frac{\nabla^{2} p}{p}
\end{gathered}
$$


and

$$
\begin{gathered}
\delta \rho_{i}^{ \pm}=\rho_{i}^{ \pm}-\sum \gamma_{i} \rho_{i}^{ \pm}, \\
\delta D_{i}=D_{i}-\sum \gamma_{i} D_{i}
\end{gathered}
$$

are deviations of the individual rate and transport coefficients from the corresponding average values.

The important point here is that, when fractions $\gamma_{i}$ instead of population densities $p_{i}$ are considered, an advective term appears (the second term in Eq. (46)) with a speed (47) opposite to the population gradient. Consider, as usual, a population range with the population density decreasing outwards (near the range edge). Then, the gradient will have the inwards direction, and the advective speed $\overrightarrow{v_{i}}$ will have the outwards direction. Therefore, according to Eqs. (46)-(47), the corresponding hydrodynamic speed $\overrightarrow{v_{i}}$ will enhance the transport of individuals outwards the population range (i.e., along the front propagation direction). On the other hand, for a shrinking population, according to Eqs. (46)-(47) the speed $\overrightarrow{v_{i}}$ would still have the outwards direction, but it would slow down the transport of individuals (because the front speed then has the inward direction). Therefore, Vlad, Cavalli-Sforza and Ross argue that the physical origin of this advective speed is the net population growth. The effect of $\overrightarrow{v_{i}}$ will be specially important on the front edge (because the speed (47) is proportional to the gradient of the total population density). Therefore, in addition to the front of the total invading population, there is an additional advancing front (a mutant cloud) of the subpopulation carrying the genetic mutation. If the mutation has appeared in an individual born on the front edge, transport will be most effective (then the two fronts are synchronized). On the other hand, if the mutation has appeared far behind the front, the mutant cloud speed will be too low to follow the invading front (and the mutation will have poor chances to spread). To apply this framework, Vlad, Cavalli-Sforza and Ross [43] considered a simplified version of Eqs. (45)(50) for a single $(n=2)$ and neutral $\left(\rho_{1}^{+}=\rho_{2}^{+} \equiv \rho^{+}\right.$, $\left.\rho_{1}^{-}=\rho_{2}^{-} \equiv \rho^{-}, D_{1}=D_{2}\right)$ mutation, logistic reproduction (i.e., $\rho^{+}-\rho^{-}=r_{L}\left(1-p / p_{\max }\right)$, see Eq. (12)) and in one dimension,

$$
\begin{gathered}
\frac{\partial p}{\partial t}=r_{L} p\left(1-\frac{p}{p_{\max }}\right)+D \frac{\partial^{2} p}{\partial x^{2}} \\
\frac{\partial \gamma}{\partial t}+\frac{\partial}{\partial x}(v \gamma)=D \frac{\partial^{2} \gamma}{\partial x^{2}}+\varepsilon \gamma
\end{gathered}
$$

where $\gamma \equiv p_{1} / p$ is the fraction of mutants and $\varepsilon \equiv \partial v / \partial x$. It is also assumed that the neutral mutation occurs at some position and time, and afterward no further mutations occur (so that $k_{12}=k_{21}=0$ ). Note that Eq. (51) is Fisher's equation (24). It has an approximate solution developed by Luther, Fisher and others, namely $[56,69]$

$$
p(x, t)=\frac{p_{\max }}{1+\exp \left[\sqrt{\frac{r_{L}}{D}} z\right]},
$$

where $z \equiv x-c_{\text {Fisher }} t$ is the coordinate at which the front is at rest, and Fisher's speed $c_{\text {Fisher }}$ is given by Eq. (23). Using this model, Vlad, Cavalli-Sforza and Ross have estimated the speed of the center of gravity of the mutant cloud as [43]

$$
c_{\text {mutant }}=\frac{c_{\text {Fisher }}}{2} .
$$

This speed is in agreement with the results of extensive numerical simulations by Edmons, Lillie and CavalliSforza, in which they obtained a cloud of mutants arising from a mutation appearing in the population and found that $c_{\text {mutant }} \simeq c_{\text {Fisher }} / 2.2[42]$.

The models reviewed in this subsection also provide a quantitative approach to Fisher's proposal on the evolution of the $\mathrm{RH}$ gene system, according to which the ancestral, African haplotype Dce underwent three major mutations which later mixed into additional haplotypes (still now observed in lesser frequencies than the other four). For example, in the range expansion from central Asia towards west Asia and eventually Europe, the mutation $D \rightarrow d$ took place, generating $d c e$ (the standard RH negative haplotype). Like the other two, dce also reaches its maximum value near the farthest point from the Asian center of the expansion (at the Basque region for $d c e$ ) [43]. Using this kind of reaction-diffusion equations, simulations can produce gene-frequency geographic distributions that can be compared to those observed today. Moreover, genetic analyses of prehistoric human remains have also become possible very recently, so in the near future it will probably become possible to compare to past gene-frequency maps (in addition to present ones). An additional application of such models is to infer the location and time of single mutational events from present gene-frequency maps [43].

\section{F. Dispersive variability}

In the simplest population models with dispersive variability, two subpopulations with different birth rates are considered: dispersers and non-dispersers, with individual number densities $U(x, y, t)$ and $V(x, y, t)$, respectively. Harris has extended a classical model due to Cook in order to take into account the effect due to the rest time $T$ between subsequent generations [23]. Then the HRD evolution equation (20) is generalized into the set

$$
\frac{\partial U}{\partial t}+\frac{T}{2} \frac{\partial^{2} U}{\partial t^{2}}=D_{d} \frac{\partial^{2} U}{\partial x^{2}}+p_{d}\left(F(U, V)+\frac{T}{2} \frac{\partial F(U, V)}{\partial t}\right),
$$

$$
\frac{\partial V}{\partial t}=\left(1-p_{d}\right) F(U, V)
$$


where

$$
F(U, V)=r_{L}(U+V)\left(1-\frac{U+V}{K}\right)
$$

is the logistic reproductive function (12), $p_{d}$ is the probability that a newborn is a disperser, $D_{d}$ is the diffusion coefficient of the dispersive subpopulation, and $K$ is the carrying capacity. Cook's model is recovered in the limit $T \rightarrow 0$, as is Fisher's model (24) in the additional limits $p_{d} \rightarrow 1$ and $V \rightarrow 0$.

Following the same method as in Sec. II.C, the front speed for Eqs. (55)-(56) has been derived and applied by Harris [23]. Other recent models with dispersive variability can be found in Ref. [22]. Future work in this direction could include a careful derivation of the evolution equations, e.g. (55)-(56), from integro-difference equations similar to (1). For example, a comparison could be made between a population made of dispersers and non-dispersers (as in Cook's and Harris' models above) and a single population in which individuals jump either a single distance or zero distance. Also, some models in the literature consider subpopulations of adults $U$ and juveniles $V$, that play the role of dispersers and nondispersers, respectively [71]. Then, $\left(1-p_{d}\right) F(U, V)$ could be replaced by $r_{L} U\left(1-\frac{U+V}{K}\right)$ in the evolution equation (56) for the juveniles, and $p_{d} F(U, V)$ could be replaced, e.g., by $-m V$ (with $m$ the subadult mortality rate) in the evolution equation (55) for the adults.

\section{THE SHAPE AND WIDTH OF FRONTS}

The shape of a front is a relevant topic in several areas, e.g. in the study of virus infection fronts (because their shapes or profiles can be directly measured experimentally [70]), in fire front research (because the width of the combustion zone is a relevant prediction [72]), etc.

The simplest reaction-diffusion evolution equation is Fisher's equation (24). Then, as mentioned in the previous section, the shape front is approximately given by Eq. (53). This result is within a few percent of that obtained from numerical simulations of Fisher's equation [56]. But Fisher's equation is obviously a very special case, and front shapes have been therefore analyzed for more general evolution equations. The rest of this section reviews such results.

\section{A. The effect of dispersive variability on the front shape}

Harris has been able to solve the shape front problem for Cook's model [23]. As mentioned in the previous section, in Cook's model the effect of the waiting time is neglected $(T \rightarrow 0)$ but two subpopulations are considered: dispersers and non-dispersers, with number densities $U(x, y, t)$ and $V(x, y, t)$, respectively, evolving according to

$$
\begin{gathered}
\frac{\partial U}{\partial t}=D_{d} \frac{\partial^{2} U}{\partial x^{2}}+p_{d} F(U, V), \\
\frac{\partial V}{\partial t}=\left(1-p_{d}\right) F(U, V) .
\end{gathered}
$$

where $p_{d} \leq 1$ is the probability that a newborn is a disperser (Fisher's equation (24) is recovered for $p_{d}=1$ ), and $F(U, V)$ is given by Eq. (57). Assuming as in Sec. II.C that $U, V \alpha \exp \left[-\lambda\left(x-c_{\mathrm{Cook}} t\right)\right]$, the front speed is easily found to be [56]

$$
c_{\mathrm{Cook}}=\sqrt{r_{L} D}\left(1+\sqrt{p_{d}}\right),
$$

and Fisher's speed (23) is recovered in the limit $p_{d} \rightarrow 1$, as it should. Harris has derived analytical formulae for the front shape by considering two cases:

(i) Low values of $p_{d}$. In this case, expansions of $U(x, y, t)$ and $V(x, y, t)$ on the small parameter $p_{d}^{1 / 2}$ are performed,

$$
\begin{aligned}
& U(x, y, t)=U_{0}(x, y, t)+p_{d}^{1 / 2} U_{1}(x, y, t)+\ldots \\
& V(x, y, t)=V_{0}(x, y, t)+p_{d}^{1 / 2} V_{1}(x, y, t)+\ldots
\end{aligned}
$$

and substitution into Eqs. (58)-(59) yields [23]

$$
\begin{aligned}
& U_{0}(x, y, t)=0, \\
& U_{1}(x, y, t)=0, \\
& V_{0}(x, y, t)=\frac{K}{1+\exp \left[z^{\prime}\right]}, \\
& V_{1}(x, y, t)=\frac{K z^{\prime} \exp \left[z^{\prime}\right]}{\left(1+\exp \left[z^{\prime}\right]\right)^{2}},
\end{aligned}
$$

where $z^{\prime} \equiv \sqrt{\frac{r_{I}}{D}}\left(x-c_{\text {Cook }} t\right)$ is the dimensionless coordinate at which the front is at rest, and the front speed $c_{\text {Cook }}$ is given by Eq. (60).

(ii) Large values of $p_{d}$. In this case, expansions of $U(x, y, t)$ and $V(x, y, t)$ on the small dimensionless parameter $\varepsilon \equiv r_{L} D c_{\text {Cook }}^{-2}$ are performed, leading to the following results for the total population density $p(x, y, t)=$ $U(x, y, t)+V(x, y, t)[23]$

$$
\begin{aligned}
p(x, y, t) & =p_{0}(x, y, t)+\varepsilon p_{1}(x, y, t)+\ldots, \\
p_{0}(x, y, t) & =\frac{K}{1+\exp \left[z^{\prime \prime}\right]}, \\
p_{1}(x, y, t) & =\frac{K p_{d} \exp \left[z^{\prime \prime}\right]}{\left(1+\exp \left[z^{\prime \prime}\right]\right)^{2}} \ln \frac{4 \exp \left[z^{\prime \prime}\right]}{\left(1+\exp \left[z^{\prime \prime}\right]\right)^{2}},
\end{aligned}
$$

where $z^{\prime \prime} \equiv z^{\prime} \sqrt{r_{L} D} / c_{\text {Cook }}$.

By plotting Eqs. (62) and (63) for several values of $p_{d}$, Harris has observed that the front shape is much steeper for low values of $p_{d}$ [23]. As would be expected, then there are fewer dispersers, so that the population towards 
the front edge is decreased and the front becomes narrower and steeper.

Before closing the discussion of Cook's model, it is worth to mention that Eq. (60) implies that populations with very few dispersers $\left(p_{d} \simeq 0\right)$ have front speeds about half the value for populations in which all individuals disperse $\left(p_{d}=1\right)$ [56]. So interestingly, even very few dispersers are able to sustain the propagation of the front without a huge reduction in its speed.

\section{B. The effect of delay times on the front width}

Fronts arise in many models involving time-delays. Their properties depend on the choice of the underlying, microscopic random walk. In order to see this, Fedotov compared two different models for the evolution of the population density $\rho(x, t)$ in one-dimensional (1D) space: model $\mathrm{A}$, discrete in time, and model $\mathrm{B}$, continuous in time, as follows [73]

model A :

$$
\rho(x, t+\tau)=\int_{-\infty}^{\infty} \rho(x+\Delta, t) \varphi(\Delta) d \Delta+\tau r f(\rho)
$$

model B :

$\partial_{t} \rho(x, t)=\lambda\left[\int_{-\infty}^{\infty} \rho(x+\Delta, t) \varphi(\Delta) d z-\rho(x, t)\right]+r f(\rho)$,

The 1D dispersion kernel $\varphi(\Delta)$ is such that $\varphi(\Delta)$ yields the probability that a particle makes a jump of length $\Delta$ ( $\Delta$ may be positive or negative). In model $\mathrm{A}$, the particle moves at regular times intervals $\tau$, whereas in model B it spends a random time between subsequent jumps with value exponentially distributed with rate $\lambda$ [74]. Also, new particles appear due to a nonlinear source term $F(\rho)=r f(\rho)$, where $r$ is the characteristic rate of reproduction. Results for logistic growth, $f(\rho)=\rho(1-\rho)$, are reviewed below. Note that model B with $\lambda=1 / \tau$ can be obtained from model A for sufficiently small values of the delay time $\tau$. Then, Fisher's equation (24) with $D=$ $\left\langle\Delta^{2}\right\rangle /(2 \tau)$ is recovered for isotropic kernels by expanding the first term in the right-hand side of Eqs. (64)-(65) up to second order in $\Delta$.

Fedotov observed that models A and B yield different front propagation speeds for the simple case of all particles jumping the same distance [73]. The speed and width of fronts have been also derived for two kernels widely used in the ecological literature, namely a Laplacian kernel,

$$
\varphi_{L}(\Delta)=\frac{1}{2 \alpha} e^{-|\Delta| / \alpha}
$$

and a Gaussian kernel,

$$
\varphi_{G}(\Delta)=\frac{1}{\alpha \sqrt{\pi}} e^{-\Delta^{2} / \alpha^{2}}
$$

Using the method explained in Sec. II.C up to second order in $\alpha$, explicit formulae for the front speeds have been derived [28],

model A :

$$
\begin{aligned}
c_{L} \simeq & \frac{\alpha}{\tau}\left[\frac{1+(1+2 \beta) \ln (1+\beta)}{(1+\beta) \ln (1+\beta)}\right]^{1 / 2} \\
& \ln \left[\frac{1+\beta+(1+\beta)^{2} \ln (1+\beta)}{1+\beta \ln (1+\beta)}\right],
\end{aligned}
$$

model A :

$$
\begin{aligned}
c_{G} \simeq & \frac{\alpha}{2 \tau}\left[\frac{1+\beta \ln (1+\beta)}{(1+\beta) \ln (1+\beta)}\right]^{1 / 2} \\
& \ln \left[(1+\beta)^{\frac{1+\beta}{1+\beta \ln (1+\beta)}}+\beta\right],
\end{aligned}
$$

model B :

$$
c_{L} \simeq \alpha \frac{2 \lambda+\gamma}{\lambda+\gamma} \sqrt{\gamma \lambda+2 \gamma^{2}}
$$

model B :

$$
c_{G} \simeq \frac{\alpha}{2} \sqrt{\frac{\lambda}{\gamma}}\left(\lambda e^{\gamma / \lambda}-\lambda+\gamma\right) .
$$

(65)

$$
\beta \equiv r \tau f^{\prime}(0) \equiv \tau \gamma
$$

The validity of these results has been checked by comparing them to the speed obtained from numerical simulations using the fast-Fourier-transform method, which makes it possible to derive precise results for integral equations with a much shorter computer time [28]. The width of fronts can be analyzed as follows. Figure 3 presents some simulated fronts according to both models. It is seen that model B yields a wider (and faster) front. From Fig. 3 we observe that there exists a inflection point $x^{*}$ such that $\partial_{x} \rho$ reaches a maximum value at $x=x^{*}$ and $\left(\partial_{x}^{2 n} \rho\right)_{x=x *}=0$ for $n=1,2,3, \ldots$ Then one has from Eq. (64) for isotropic kernels [i.e., $\varphi(\Delta)=\varphi(-\Delta)$ ],

$$
\rho\left(x^{*}, t+\tau\right)=\rho\left(x^{*}, t\right)+\left.\operatorname{\tau rf}(\rho)\right|_{x=x^{*}} .
$$

We also have, in the limit $\tau \ll t$,

$$
\rho\left(x^{*}, t+\tau\right) \simeq \rho\left(x^{*}, t\right)+\left.\tau \partial_{t} \rho\right|_{x=x^{*}},
$$

so that

$$
\left.\left.\partial_{t} \rho\right|_{x=x^{*}} \simeq \operatorname{rf}(\rho)\right|_{x=x^{*}}
$$

and the same result holds for model B with $\lambda=1 / \tau$. We change into a frame moving with the front by defining the coordinate $z \equiv x-c t$. For $x=x^{*}$ we get $-\left.c \partial_{z} \rho\right|_{z=z^{*}} \simeq$ $\left.r f(\rho)\right|_{x=x^{*}}$. The width of the front $L$ is given by 


$$
L^{-1}=-\left.\left.\partial_{z} \rho\right|_{z=z^{*}} \simeq \frac{r}{c} f(\rho)\right|_{z=z^{*}}
$$

In Fig. 4 we compare this prediction to the results of numerical simulations for a logistic reactive process $\left(\left.f(\rho)\right|_{z=z^{*}}=f\left(\frac{1}{2}\right)=\frac{1}{4}\right)$. The front width is estimated from the simulated profiles by fitting a straight line to the central range $(\rho \simeq 1 / 2$ ) of profiles such as those in Fig. 3 and, as mentioned above, the front width is estimated as the inverse of the slope of the fitted line. From Fig. 4, we see that there is good agreement with the theoretical prediction given by Eq. (76). Note that from Eq. (76), the front speed is proportional to the front width in both models. A higher value of the delay time corresponds to a slower, narrower front, as was to be expected. Figure 4 shows that, the higher the value of $\tau$, the more error results from using model $\mathrm{B}$ as an approximation to model A, also as expected. This error is higher than $20 \%$ in Fig. 4 and is the same for the front speed and the front width, because they are proportional to each other [see Eq. (76), which makes it possible to determine the speed from any value of the front width in Fig. 4]. Therefore, when using model $\mathrm{B}$ as an approximation to model $\mathrm{A}$, one should previously see if the error, computed in the way explained here and illustrated by Fig. 4, is negligible or not for the parameter values used.

\section{COMBUSTION FLAMES}

Flame propagation models are based on non-sequential reaction-diffusion equations similar to (24), where the source function $F$ has a strong dependence on temperature $T$ [75]. A lot of work has been devoted to obtain the propagation speed of flames by means of combustion modeling through a variety of approaches and numerical simulation strategies. The problem is indeed daunting since an adequate account of the combustion process must address transport properties, the chemistry of the reacting mixture, and their coupling [76,77]. Very recently, numerical simulations of highly complex models have provided results which have been successfully validated against measurements obtained from experiments $[78,79]$. However, these types of studies require a strong computational effort and do not yield equations for the influence of the system parameters on the flame speed. In contrast, the application of some levels of simplification to the governing equations leads to simple models from which estimates for the propagation speed of the flame are obtained by employing a variety of techniques [80-82]. Here we review some recent developments on this field [29-34], which provide good estimates for the front propagation speed in some combustion processes.

\section{A. Background flow at rest, no mass diffusion and constant transport coefficients}

In premixed gas flames, the fuel, oxidant and inert gases are mixed on the molecular scale before combustion is initiated [83]. Here we restrict our attention to onedimensional (1D) models of laminar premixed gas flames with a single-step reaction mechanism

$$
R(\text { reactants }) \rightarrow P(\text { products })
$$

where the reaction rate $w$ (i.e., the normalized reactant consumption rate due to the chemical reaction) at absolute temperature $T$ is given by an Arrhenius expression

$$
w(T)=A e^{-\frac{E a}{R T}}
$$

with activation energy per mole $E_{a}$, universal gas constant $R$, and preexponential factor $A$ (i.e., the inverse of a characteristic reaction time) [84].

Under these assumptions and neglecting mass diffusion, advection, radiative heat losses and conductive heat losses through the boundaries, the model reduces to the following balance equations of energy and fuel density $\rho_{F}$ $[32]$

$$
\begin{gathered}
\frac{\partial \theta}{\partial t^{\prime}}=\frac{\partial^{2} \theta}{\partial r^{\prime 2}}+\rho^{\prime}\left(e^{-\frac{1}{\theta}}-e^{-\frac{1}{\theta_{0}}}\right), \\
\frac{\partial \rho^{\prime}}{\partial t^{\prime}}=-C \rho^{\prime}\left(e^{-\frac{1}{\theta}}-e^{-\frac{1}{\theta_{0}}}\right),
\end{gathered}
$$

where we have used the dimensionless variables and parameters

$$
\begin{gathered}
\theta \equiv T \frac{R}{E_{a}}, \\
r^{\prime} \equiv r \sqrt{\frac{R Q A}{\widetilde{D} E_{a} c_{p}}}, \\
t^{\prime} \equiv t \frac{R Q A}{c_{p} E_{a}}, \\
\rho^{\prime} \equiv \frac{\rho_{F}}{\rho}, \\
C \equiv \frac{c_{p} E_{a}}{R Q},
\end{gathered}
$$

where $T$ is the absolute temperature, $r$ is the radial coordinate, $t$ is the time, $Q$ is the heat produced by the combustion reaction per unit mass of fuel, $c_{p}$ is the specific heat of the mixture at constant pressure, $\rho$ is the density of the mixture (which is constant, since here we 
neglect convection; see [33]) and $\widetilde{D} \equiv \lambda /\left(c_{p} \rho\right)$ is the heat diffusivity, where $\lambda$ is the thermal conductivity (assumed constant in this subsection).

Equation (80) corresponds to the consumption of fuel, and prevents the temperature from increasing without bound. The last term within the second parentheses in Eqs. (79)-(80) is the so-called 'cold boundary layer' heat loss term, and it ensures steadiness $\left(\frac{\partial \theta}{\partial t^{\prime}}=0\right.$ and $\left.\frac{\partial \rho^{\prime}}{\partial t^{\prime}}=0\right)$ if all points of the system are at room temperature $(\theta=$ $\left.\theta_{0}\right)[32,85]$.

This framework is essentially the model of a premixed gas flame assumed by Zeldovich and Frank-Kamenentskii $[75,86]$.

Note that the dimensionless front speed $v=d r^{\prime} / d t^{\prime}$ is related to its speed $c=d r / d t$ as

$$
v=c \sqrt{\frac{C}{\widetilde{D} A}}
$$

The boundary conditions are $\theta \rightarrow \theta_{0}$ (room temperature), $\rho^{\prime} \rightarrow 1$ at $r \rightarrow \infty$, and $\theta=\theta_{\max }$ (maximum temperature), $\rho^{\prime} \rightarrow 0$ at $r=0$. The latter condition would break down if heat losses were included (the solution would then be a pulse rather than a front [32]). A recent mathematical procedure has reduced the system of two partial differential equations (PDEs) (79)-(80) into a single reaction-diffusion equation of a single variable [32]. For this purpose, the main requirement is that both thermal and mass gradients reach non-zero values only in the narrow region where the front arises. This leads to the following equations, that have been tested by numerical integrations of the system (79)-(80) [32],

$$
\theta=\theta_{0}+\frac{\left(1-\rho^{\prime}\right)}{C}
$$

and

$$
\theta_{\max }=\theta_{0}+\frac{1}{C}
$$

These equations lead to the final result that the model (79)-(80) (which neglects mass diffusion, advection, radiative losses and conductive losses through the boundaries) is reduced to a single PDE, namely [32]

$$
\frac{\partial n}{\partial t^{\prime}}=\frac{\partial^{2} n}{\partial r^{\prime 2}}+F(n)
$$

where a new dimensionless variable $n$ is defined as

$$
n=\frac{\theta-\theta_{0}}{\theta_{\max }-\theta_{0}}
$$

This implies that the variable $n$ varies from 0 to 1 , which is a requirement of some of the methods reviewed below. The reaction term in Eq. (89) reads [32]

$$
F(n)=C(1-n)\left(e^{-\frac{1}{\theta_{0}+\left(\theta_{\max }-\theta_{0}\right) n}}-e^{-\frac{1}{\theta_{0}}}\right) .
$$

Equation (91) satisfies the condition $F(n) \geqslant 0$ for $0 \leqslant$ $n \leqslant 1$ with $F(n=0)=0$ and $F(n=1)=0$, which are also necessary conditions for applying some of the methods here reviewed.

Equation (89) is a non-sequential reaction-diffusion equation but with a source function, Eq. (91), that differs from the logistic function (12) applied in many biophysical problems. The nonlinearities in Eq. (91) avoid an exact result for the front speed, in contrast to the exact result (23) valid for the logistic source function (12). This has lead to several authors to derive bounds on the propagation speed of the flame front. In this subsection we review the main expressions derived, both for lower $\left(v_{L B}\right)$ and for upper bounds $\left(v_{U B}\right)[32,29]$.

\section{Zeldovich-Frank-Kamenentskii (ZFK) lower bound}

The method employed by Zeldovich and FrankKamenentskii assumes a large thermal gradient in the reaction zone. Then, the heat conduction term in Eq. (79) dominates over the temporal derivative term, which corresponds to heat convection in a frame moving with the flame $(z \equiv r-c t)$ and is positive in the absence of heat losses. Therefore, using dimensional variables $[75,86]$,

$$
0 \leqslant \frac{d}{d z}\left(\lambda \frac{d T}{d z}\right)+Q A \rho_{F}\left(e^{-\frac{E_{a}}{R T}}-e^{-\frac{E_{a}}{R T_{0}}}\right) .
$$

The integration of Eq. (92) in the variable $y \equiv \lambda \frac{d T}{d z}$ (so that $\frac{1}{d z}=\frac{y}{\lambda d T}$ ) from the boundary of the reaction zone $\left(T \simeq T_{0}\right)$ to the burned zone $\left(y \simeq 0, T=T_{\max }\right)$ leads to [75]

$$
\lambda \frac{d T}{d z} \geqslant \sqrt{2 Q \lambda A \rho_{F} \int_{T_{0}}^{T_{\max }}\left(e^{-\frac{E_{a}}{R T}}-e^{-\frac{E_{a}}{R T_{0}}}\right) d T .}
$$

In addition, it is assumed that the heat flux at the reaction zone must equal the energy released by combustion

$$
\lambda \frac{d T}{d z}=Q \rho c
$$

Combining Eq. (93) with (94) and using (87) leads to a lower bound for the propagation speed $v$ of the front flame

$$
c \geqslant \sqrt{\frac{2 \lambda}{Q \rho} A\left(1-C\left(\theta-\theta_{0}\right)\right) \int_{T_{0}}^{T_{\max }}\left(e^{-\frac{E_{a}}{R T}}-e^{-\frac{E_{a}}{R T_{0}}}\right) d T .}
$$

Using dimensionless variables and Eqs. (85) and (88), this lower bound reads

$$
v \geqslant v_{L B}^{Z F K}=\sqrt{2 \int_{0}^{1} F(n) d n .}
$$




\section{Kolmogorov-Petrovski-Piskunov (KPP) lower bound}

This method is based on the linearization of Eq. (89) after introducing the variable $z=r^{\prime}-v t^{\prime}$ with a solution of the type $n(z)=n_{0} e^{-\zeta z}$ with $\zeta>0$ for $t \rightarrow \infty$ [87]. It therefore corresponds to the linearization method reviewed in Sec. II. C. This leads to a second-order equation for $\zeta$,

$$
\zeta^{2}+v \zeta+\left(\frac{d F(n)}{d n}\right)_{n=0}=0,
$$

and the condition that $\zeta$ is real implies that the propagation speed $v$ must be greater than the lower bound $v_{L B}^{K P P}$

$$
v \geqslant v_{L B}^{K P P}=2 \sqrt{\left(\frac{d F(n)}{d n}\right)_{n=0}} .
$$

However, as we shall review below, numerical integrations of the set (79)-(80) have shown that for the function (91) the bound (98) yields estimates that are several orders of magnitude below the correct speed [32].

\section{Benguria-Cisternas-Depassier (BCD) lower bound}

Benguria et al. [85] have developed a variational technique which is very useful for providing estimates for the front speed in a wide variety of $1 \mathrm{D}$ reaction-diffusion equations. For the particular case of Eq. (89) with Eq. (91), the lower bound $v_{L B}^{B C D}$ for the dimensionless propagation speed $v$ is

$$
v \geqslant v_{L B}^{B C D}=\frac{4 \sqrt{i}}{2 i+1} \frac{\left(\int_{0}^{1} F(n) d n\right)^{i+1 / 2}}{\int_{0}^{1}\left(\int_{x}^{1} F(n) d n\right)^{i} d x},
$$

and this bound holds for any value of $i$ such that $1 / 2 \leqslant$ $i \leqslant 1$. It is important to stress that Eq. (99) applies only for those systems with $\partial n / \partial r^{\prime} \leqslant 0$. This requirement reduces the application of Eq. (99) to fronts since in the propagation of pulses, either the radiative or conductive cooling lead to regions with $\partial n / \partial r^{\prime} \geqslant 0$. Another condition implicitly assumed in deriving Eq. (99) is that $F(n=0)=0, F(n=1)=0$ and $F(n) \geqslant 0$ for $0 \leqslant n \leqslant 1$.

\section{Aronson-Weinberger ( $A W)$ upper bound}

The upper bound $v_{U B}^{A W}$ derived by Aronson and Weinberger [88] follows from mathematical analysis applied to the phase space of Eq. (89). As explained in the KPP method above, in the front reference frame $\left(z=r^{\prime}-v t^{\prime}\right)$ Eq. (89) becomes

$$
n_{z z}^{\prime \prime}+v n_{z}^{\prime}+F(n)=0
$$

where the symbol ' denotes derivative relative to $z$. Introducing $q \equiv n$, this equation is equivalent to the system

$$
\begin{aligned}
& q^{\prime}=p, \\
& p^{\prime}=-v p-F(q) .
\end{aligned}
$$

The functions $p(z), q(z)$ corresponding to a solution of Eq. (100) give a trajectory in the $q-p$ plane (or, as it is usually called, the phase space) such that

$$
\frac{d p}{d q}=-v-\frac{F(q)}{p} .
$$

Plane wave solutions to Eq. (100) such that $n \rightarrow 0$ as $z \rightarrow \infty$ correspond to trajectories in the phase space through the point $(p, q)=(0,0)$. For such solutions with bounded support for $t=0$, the theorems due to Aronson and Weinberger rigorously prove the existence of a minimum speed $v_{U B}^{A W}$ given by $[88,89]$

$$
v \leqslant v_{U B}^{A W}=2 \sqrt{\sup _{n \in[0,1]}\left(\frac{F(n)}{n}\right)},
$$

where the supremum of the function $F(n) / n$ is taken over $n \in[0,1]$.

It is very important that the unique requirement imposed by Aronson and Weinberger in order to derive Eq. $(102)$ is $F(n=0)=0$ and $F(n=1)=0$, with $F(n) \geqslant 0$ for $0 \leqslant n \leqslant 1$. This is very encouraging since it implies that Eq. (102) is suitable for being applied to a large variety of cases. Indeed, recently it has been applied not only to fronts but also to flame pulses, which are physically more realistic (because flame extinguishment due to heat losses is included) but mathematically much more complicated [32-34].

\section{Benguria-Depassier (BD) upper bound}

Benguria and Depassier [81] applied a variational technique to derive the following upper bound for the dimensionless propagation speed $v$,

$$
v \leqslant v_{U B}^{B D}=2 \sqrt{\sup _{n \in[0,1]}\left(\frac{d F(n)}{d n}\right)},
$$

which is valid for any function $F(n)$ that satisfies $F(n=$ $0)=0, F(n=0)=1$ with $F(n) \geqslant 0$ for $0 \leqslant n \leqslant 1$ and $\partial n /\left.\partial r^{\prime}\right|_{n=0}=0$ and $\partial n /\left.\partial r^{\prime}\right|_{n=1}=0$ with $\partial n / \partial r^{\prime} \leqslant 0$ for $0 \leqslant n \leqslant 1[81]$.

\section{Benguria-Depassier-Méndez (BDM) upper bound}

Finally, Benguria, Depassier and Méndez [29] have recently found an upper bound by using the same variational technique as for the lower bound $v_{L B}^{B C D}$. Their 
result is valid for any function $F(n)$ that satisfies $F(n=$ $0)=0, F(n=1)=0$ and $F(n) \geqslant 0$ for $0 \leqslant n \leqslant 1$. It also requires that $\partial n / \partial r^{\prime} \leqslant 0$. This bound reads [29]

$$
v \leqslant v_{U B}^{B D M}=\sup _{n \in[0,1]}\left[\phi \frac{F(n)}{n}+\frac{1}{\phi}\right],
$$

with $\phi$ any positive constant. By choosing $\phi=$ $1 / \sup _{n \in[0,1]} \sqrt{F(n) / n}$, Eq. (104) corresponds to the classical Aronson and Weinberger bound (102).

\section{Propagation speeds}

Figure 5 shows a comparison of the five bounds described above, as well as the propagation speeds obtained by means of numerical integrations (circles) of the full model that consists of the system of two PDEs (79)-(80), for several values of the room temperature $\theta_{0}[32]$. In comparison with the full model, the best upper bound is that due to Aronson and Weinberger (102). Concerning lower bounds, Eq. (99) with $i=1$ provides the best estimate to the simulations. Both upper and lower bounds give a realistic variation of the speed, and they jointly lead to correct predictions for its order of magnitude. Although a direct comparison to experiment requires much more complicated, purely numerical approaches (i.e., a large set of differential equations involving a lot of chemical reactions and additional transport phenomena [76-79]), relatively simple models yield analytical bounds (such as Fig. 5) that are a useful guide of the kind of dependencies that should be expected, as well as a practical check of more complicated numerical codes.

\section{B. Background flow, mass diffusion and transport coefficients dependent on temperature}

The previous subsection has analyzed a premixed gas flame with a single-step reaction in a fluid at rest, without mass diffusion and with constant transport coefficients. Here we extend the model to include 1) advection, 2) mass diffusion and 3) the dependence of transport coefficients on temperature [33].

Advection arises through the presence of a background flow or by the effect of buoyancy (in both cases, the influence of advection on the front speed may not be negligible [90]). Mass diffusion, if the species have different specific heats, leads to heat exchanges that may also influence the propagation speed of the front [33]. Finally, temperature greatly varies in combustion processes, which implies a variation of both mass and heat transport coefficients important enough to modify the propagation speed $c$.

These three effects (advection, mass diffusion and transport coefficients as a function of temperature) are now included in the evolution equations for dimensionless temperature $\theta(81)$ and fuel density $\rho^{\prime}(84)[33,34,91]$,

$$
\begin{aligned}
\frac{\partial \theta}{\partial t^{\prime}}+\left(\frac{C}{\widetilde{D}_{0} A}\right)^{1 / 2} U \frac{\partial \theta}{\partial r^{\prime}}= & \frac{\partial}{\partial r^{\prime}}\left(\frac{\lambda}{\lambda_{0}} \frac{\partial \theta}{\partial r^{\prime}}\right) \\
& +\operatorname{Le}_{0} \frac{D}{D_{0}} \frac{\Delta c_{p}}{c_{p}} \frac{\partial \rho^{\prime}}{\partial r^{\prime}} \frac{\partial \theta}{\partial r^{\prime}} \\
& +\rho^{\prime}\left(e^{-\frac{1}{\theta}}-e^{-\frac{1}{\theta_{0}}}\right)
\end{aligned}
$$

$$
\begin{aligned}
\frac{\partial \rho^{\prime}}{\partial t^{\prime}}+\left(\frac{C}{\widetilde{D}_{0} A}\right)^{1 / 2} U \frac{\partial \rho^{\prime}}{\partial r^{\prime}}= & \frac{\partial}{\partial r^{\prime}}\left(\operatorname{Le}_{0} \frac{D}{D_{0}} \frac{\partial \rho^{\prime}}{\partial r^{\prime}}\right) \\
& -C \rho^{\prime}\left(e^{-\frac{1}{\theta}}-e^{-\frac{1}{\theta_{0}}}\right)
\end{aligned}
$$

where the dimensionless time $t^{\prime}$ is defined by Eq. (83), the parameter $C$ by Eq. (85) and the dimensionless radial coordinate $r^{\prime}$ now follows

$$
r^{\prime} \equiv r \sqrt{\frac{R Q A}{\widetilde{D}_{0} E_{a} c_{p}}},
$$

where $\widetilde{D}_{0}$ is the heat diffusivity at room temperature and the other variables are defined below Eq. (82). In Eqs. (105)-(106) $U$ is the velocity field of the background flow, $c_{p}$ is the specific heat at constant pressure of the mixture $\left(\rho c_{p}=c_{p, F} \rho_{F}+c_{p, N F} \rho_{N F}\right), \Delta c_{p}=c_{p, F}-c_{p, N F}$, where the subindex $F$ stands up for fuel, and $N F$ for non-fuel (inert gases, oxidizers and products), and

$$
\mathrm{Le}_{0}=\frac{D_{0}}{\widetilde{D}_{0}}
$$

is the Lewis number at room temperature, where $D_{0}$ is the mass diffusivity at room temperature. The Lewis number is a key parameter in flame dynamics, a fact noted both experimentally and numerically [92], with a wide variety of studies on flame dynamics carried out for different values of Le (see, e.g., [90,93]). Thermal conductivity $\lambda$ and mass diffusivity $D$ now follow

$$
\lambda=\lambda_{0}\left(\frac{T}{T_{0}}\right)^{\alpha}, D=D_{0}\left(\frac{T}{T_{0}}\right)^{\beta},
$$

where $T$ is the absolute temperature, $T_{0}$ is the room temperature and $\lambda_{0}=\lambda\left(T=T_{0}\right)$. Zeldovich et al. [75] use Eq. (109) in the analysis of combustion processes with $\alpha \simeq \beta \simeq 0.6$.

Eqs. (105)-(106) implicitly assume a constant value for the total density $\rho$, which holds either for the particular case of a background flow at rest (i.e., $U=0$ ) [33] or for the case of uniform advection (i.e., $U=$ constant). Note, however, that in the following subsection we also analyze the case of convection produced from density changes across the flame front. Then, and following Vladimirova et al. [31], we assume as a first approximation that density changes modify the flow velocity but not appreciably the diffusion terms in Eqs. (105)-(106). These diffusion terms, indeed, lead to a small correction 
(circles in Fig. 6), so the role of convection on these small, diffusion terms is a second-order effect on the flame speed and can be neglected [31].

The nonlinear term $\partial \rho^{\prime} / \partial r^{\prime} \partial \theta / \partial r^{\prime}$ in Eq. (105) corresponds to the diffusion of fuel into the burned region (i.e., that where fuel has been consumed by the combustion reaction). Since fuel and non-fuel have different specific heats (i.e., $c_{p, F} \neq c_{p, N F}$ ), this term does not vanish. Here we assume that $c_{p, F}>c_{p, N F}$, which is obviously expected to reduce the flame temperature and slow down the flame speed in comparison with the $c_{p, F}=c_{p, N F}$ case (a speed decrease is indeed observed in Fig. 6).

The set (105)-(106) is in fact a generalization of Eqs. (79)-(80) to include advection $(U \neq 0)$, mass diffusion $\left(\mathrm{Le}_{0} \neq 0\right)$ and transport coefficients dependent on temperature $(\alpha \neq 0, \beta \neq 0)$. Recent work has reduced the coupled set (105)-(106) to a single equation with a single variable, namely $[33,34]$

$$
\begin{aligned}
\frac{\partial n}{\partial t^{\prime}}= & -U\left(\frac{C}{\widetilde{D}_{0} A}\right)^{1 / 2} \frac{\partial n}{\partial r^{\prime}}+A(n) \frac{\partial^{2} n}{\partial r^{\prime 2}} \\
& +B(n)\left(\frac{\partial n}{\partial r^{\prime}}\right)^{2}+F(n)
\end{aligned}
$$

where,

$$
\begin{gathered}
A(n)=\left(1+\frac{n}{C \theta_{0}}\right)^{\alpha} \\
B(n)=\frac{\alpha}{C \theta_{0}}\left(1+\frac{n}{C \theta_{0}}\right)^{\alpha-1}-\operatorname{Le}_{0} \frac{\Delta c_{p}}{c_{p}}\left(1+\frac{n}{C \theta_{0}}\right)^{\beta}
\end{gathered}
$$

and, again, $n$ is defined by Eq. (90) and $F(n)$ follows the non-linearity (91). Eq. (110) arises by assuming a linear dependence of temperature on fuel density, see Eq. (87), which is again obtained after neglecting the contributions of both temperature and fuel density first and second order gradients compared to the combustion reaction term in the integration of Eqs. (105)-(106) from $t^{\prime}=0$ to $t^{\prime}$ [34]. Thus, Eq. (110) is indeed an approximation of the full combustion model of a laminar premixed gas flame that consists of two coupled PDEs (105)-(106). It can in principle break down for flames with a reaction zone which is sufficiently wide, i.e. for sufficiently smooth profiles of temperature and fuel density. However, the validity of Eq. (110) for realistic parameter values has been tested by means of numerical integrations of the full model (110)-(112) [32-34].

Note that Eq. (89) corresponds to a limiting case of Eq. (110) for $\mathrm{Le}_{0}=0$ (no mass diffusion), $U=0$ (no advection) and $\alpha=0$ (constant thermal conductivity).

\section{The effect of convection}

Neglecting mass diffusion (i.e., $\mathrm{Le}_{0}=0$ ), for nonArrhenius reaction rates and for constant values of trans- port coefficients, Vladimirova et al. [31] have recently investigated the effects of using two relevant velocity fields on the propagation speed of the combustion flame: 1) the simple case of a uniform value for the background field $U$, and 2) the case of taking the effect of the thermal expansion through the flame thickness into account. The first case implicitly assumes an incompressible (constantdensity) fluid. Then the dimensionless front speed $v$ simply becomes the sum of the dimensionless flow velocity $\left(C /\left(\widetilde{D}_{0} A\right)\right)^{1 / 2} U$ and the front propagation speed obtained for the particular case of no-convection $(U=0)$.

The second case analyzed by Vladimirova et al. considers a compressible (variable-density) fluid. In other words, density changes due to thermal differences across the flame front are taken into account. The mass balance equation in the front reference frame, namely $\rho(U-c)=$ $\rho_{0}\left(U_{0}-c\right)$, leads to the following velocity field $U[31]$

$$
\begin{aligned}
\left(\frac{C}{\widetilde{D}_{0} A}\right)^{1 / 2} U= & \left(\frac{\mathrm{Pe}}{\mathrm{Da}} C\right)^{1 / 2} \frac{U}{U_{0}}= \\
& \left(\frac{\mathrm{Pe}}{\mathrm{Da}} C\right)^{1 / 2}\left[\frac{\rho_{0}}{\rho}+\frac{c}{U_{0}}\left(1-\frac{\rho_{0}}{\rho}\right)\right],
\end{aligned}
$$

where $\rho_{0}$ and $U_{0}$ are the total density and the flow velocity at the unburned region, respectively, and are used to introduce the Péclet number $\mathrm{Pe}=U_{0} L / \widetilde{D}_{0}$, with $L$ a characteristic length scale, and the Damköhler number $\mathrm{Da}=L A / U_{0}$. The Péclet number can be expressed in terms of the Reynolds number $\operatorname{Re}=U_{0} L / \nu_{0}$, by using the Prandtl number $\operatorname{Pr}=\nu_{0} / \widetilde{D}_{0}$, where $\nu_{0}$ is the kinematic viscosity at a reference point. In Eq. (113), note that $c$ is the propagation speed of the flame which, indeed, is the value we seek to estimate, so in this case the simulation of the full model (105)-(106) requires an iterative process [34].

By assuming a constant-pressure process, densities in Eq. (113) are easily related to temperatures and [34]

$$
\left(\frac{C}{\widetilde{D}_{0} A}\right)^{1 / 2} U=\left(\frac{\mathrm{Pe}}{\mathrm{Da}} C\right)^{1 / 2}\left[1+\frac{n}{C \theta_{0}}\left(1-\frac{v}{u_{0}}\right)\right],
$$

where $n$ is again defined by Eq. (90), $v$ is the dimensionless propagation speed $\left(v \equiv c \sqrt{C} / \sqrt{\widetilde{D}_{0} A}\right)$ and we have introduced the dimensionless reference flow as $u_{0} \equiv \sqrt{C} U_{0} / \sqrt{\widetilde{D}_{0} A}$.

Vladimirova et al. [31] obtain the flame speed by the linearization method employed in the KPP lower bound procedure detailed above. Recent work [34] has analyzed the same background fields for an Arrhenius reaction rate (so that the KPP method breaks down, as explained above) and by including the effect of both mass diffusion (i.e., Le $\neq 0$ ) and transport coefficients as a function of temperature. 


\section{Upper and lower bounds}

The variational method developed by Benguria et al. [81] has been recently applied to Eq. (110) in order to obtain bounds on the propagation speed of flames [34]. For $\beta$ below a critical value $\beta_{c}$ given by

$$
\begin{aligned}
\operatorname{Le}_{0} \frac{\Delta c_{p}}{c_{p}}\left(1+\frac{1}{C \theta_{0}}\right)^{\beta_{c}}= & \left(1+\frac{1}{C \theta_{0}}\right)^{\alpha-1} \\
& {\left[1+(\alpha+1) \frac{1}{C \theta_{0}}\right] } \\
& +\left(\frac{C}{\widetilde{D}_{0} A}\right)^{1 / 2},
\end{aligned}
$$

where

$$
\phi=\sup _{n \in[0,1]} \sqrt{\frac{F(n)}{n}},
$$

the speed satisfies $v \leqslant v_{U B}$, where the upper bound $v_{U B}$ is $[34]$

$$
\begin{aligned}
v_{U B}= & \sup _{n \in[0,1]}\left\{\frac{F(n)}{n \phi}+\phi\left(1+\frac{n}{C \theta_{0}}\right)^{\alpha-1}\left[1+\frac{(\alpha+1) n}{C \theta_{0}}\right]\right. \\
& -\phi \operatorname{Le}_{0} \frac{\Delta c_{p}}{c_{p}}\left(1+\frac{n}{C \theta_{0}}\right)^{\beta} n \\
& \left.+\left(\frac{\mathrm{Pe}}{\mathrm{Da}} C\right)^{1 / 2}\left(1+\frac{n}{C \theta_{0}}\right)\right\} .
\end{aligned}
$$

Equation (117) only holds if $F(0)=0, F(1)=0$, $F(n) \geqslant 0$ for $0 \leqslant n \leqslant 1$ and $\partial n / \partial r^{\prime} \leqslant 0$. It is important to notice that the last condition applies for fronts but not for pulses. This implies that the variational method as developed by Benguria et al. $[81,85,29]$ is not able to provide limits for the propagation speed when Eq. (110) is generalized to include energy losses (that case is considered in Refs. [32,33]). Note that Eq. (117) for $\mathrm{Le}_{0}=0$, $U_{0}=0$ and $\alpha=0$ reverts to the Aronson and Weinberger upper bound (102), which can be applied both to front and to pulse solutions [32-34].

However, the variational method developed by Benguria et al. $[81,85,29]$ may be also used for finding the lower bound of the propagation speed of the flame front predicted by Eq. (110). By doing so, Pujol et al. [34] derive $v \geqslant v_{L B}$, with

$$
\begin{aligned}
v_{L B}= & \frac{\sqrt{\frac{C}{D_{0} A}}}{\int_{0}^{1} g(n) d n}\left[2 \int _ { 0 } ^ { 1 } d n \left(F ( n ) g ( n ) \left[-g^{\prime}(n)\left(1+\frac{n}{C \theta_{0}}\right)^{\alpha}\right.\right.\right. \\
& \left.\left.\left.-\operatorname{Le}_{0} \frac{\Delta c_{p}}{c_{p}} g(n)\left(1+\frac{n}{C \theta_{0}}\right)^{\beta}\right]\right)^{1 / 2} \int_{0}^{1} U g(n) d n\right] .
\end{aligned}
$$

where $g(n)$ is an arbitrary positive function with $g^{\prime}(n)=d g(n) / d n$ that satisfies the requirement
$-g^{\prime}(n)\left(1+\frac{n}{C \theta_{0}}\right)^{\alpha}-\operatorname{Le}_{0} \frac{\Delta c_{p}}{c_{p}} g(n)\left(1+\frac{n}{C \theta_{0}}\right)^{\beta}>0$. We evaluate $v_{L B}$ by using the following trial function $g(n)$

$$
g=\sqrt{1-n}
$$

that satisfies the requirement stated above when $\alpha \geq \beta$ for $\mathrm{Le}_{0} \Delta c_{p} / c_{p}=0.5$.

\section{Propagation speeds}

Figure 6 shows the dependence of the propagation speed of combustion flames on the Lewis number $\mathrm{Le}_{0}$ for a case without background flow $(U=0)$ and constant values for the transport coefficients $(\alpha=0, \beta=0)$. There is agreement between the simulations of the coupled PDEs (105)-(106) (circles) and the lower and upper bounds (118) and (117).

Figure 7 analyzes the dependence of the propagation speed of combustion flames on $\alpha$, so we neglect the effect of mass diffusion in Fig. 7 (i.e., $\mathrm{Le}_{0}=0$, so the value of $\beta$ is irrelevant). Figure 7 compares the dimensionless speed $v$ obtained from the full model (105)-(106) (circles) with the estimates provided by Eqs. (117) (solid lines) and (118) (dashed line). In Figure 7, $\alpha$ ranges from $\alpha=0$ (constant thermal conductivity) to $\alpha=1(\lambda \propto T)$ for two cases: (i) a background flow at rest $(U=0)$ and (ii) a background flow with $\operatorname{Re}=1000$. The values of the parameters are $C=2.5, \theta_{0}=0.072$, which lead to a room temperature $T_{0}=300 \mathrm{~K}$ and a flame temperature $T_{\max }$ $=1966 \mathrm{~K}$, Prandtl number $\mathrm{Pr}=0.7, \Delta c_{p} / c_{p}=0.5$, Da $=3.3 \times 10^{8}$ and $A=3.3 \times 10^{7} \mathrm{~s}^{-1}$ [34]. As expected, the dimensionless propagation speed increases as $\alpha$ increases (heat conduction increases). The agreement between the simulations of the coupled PDEs (105)-(106) (circles) and the lower (118) and upper bounds (117) is better at low values of $\alpha$.

The effect of mass diffusion on the flame propagation speed for $\alpha \neq 0$ and $\beta \neq 0$ is shown in Figure 8, where $\mathrm{Le}_{0}=1$ and $\mathrm{Re}=0$ (background flow at rest), and the other parameter values as above. Solid lines in Fig. 8 shown the values of the dimensionless propagation speed as a function of $\alpha$ and $\beta$, whereas dashed lines correspond to the upper bound values derived from Eq. (117). The lower bound is not shown for clarity (and because if holds only for $\alpha \geq \beta$ ). Results for the very same case but with advection $(\mathrm{Re}=1000)$ are shown in Fig. 9. The upper bounds make it possible to predict the correct order of magnitude of the flame front speed, as well as its variation with the system parameters.

\section{Other combustion processes}

(i) Here we have focused on front propagation, but the AW method has also been shown to give reasonable 
results for all of the cases above but considering pulse propagation instead (due to heat losses) [32-34].

(ii) Front propagation in heterogeneous media for different types of reaction rate functions are tackled by Xin [80]. Xin applies variational principles similar to those developed by Benguria, Depassier and others stated above [81] in order to obtain bounds for periodic media [80].

(iii) The analysis of random media is of great importance since it includes the effect of turbulent flows. Then, the reaction-diffusion-advection equation contains a random velocity of turbulent spectrum and zero mean ensemble. Combustion research analyzing turbulent flows in the limiting cas of thin flames compared to the largest scale of the turbulence has reached a result for the front speed of turbulent flames $S_{T}$ that reads

$$
S_{T}=S_{L} \frac{A_{T}}{A_{L}}
$$

where $S_{L}$ is the laminar front speed, $A_{T}$ is the surface area of the wrinkled front and $A_{L}$ is the cross-sectional area with respect to the direction of front propagation $[80,30]$. Eq. (120) is valid only if diffusive effects are small compared to fluid dynamics effects on the front speed. Then, the flame front is approximated by a surface wrinkled by the turbulent velocity. However, and as noted by Xin, the dependence of $A_{T}$ on the background turbulent flow is nontrivial [80]. Note that in turbulent media, the flame has different characteristics depending on a variety of chemical and background flow scales. Thus, flamelet combustion corresponds to chemical reaction occurring at fast time scales and short length scales relative to the turbulence. In some laminar regimes, much of the interaction between combustion and turbulence is decoupled, greatly simplifying the modeling task [92].

(iv) Here we have considered premixed gas flames. In nonpremixed flames, two nonpremixed reactants (fuel $\mathrm{F}$ and oxidant $\mathrm{O}$ ) react to form product $\mathrm{P}$ [94]. The exothermic energy released by the chemical reaction causes variations in density that, in the presence of a gravity field, may induce a buoyancy force. The behavior of this type of processes is usually simulated by complex numerical algorithms based on the governing equations of energy and mass and no further simplifications are made in order to derive limits for the propagation speed of the front.

(v) Flame spread over beds of solid (or liquid) fuel is essentially a $2 \mathrm{D}$ process. Indeed, the heat flux from the front flame vaporizes the fuel from the surface of the solid which will eventually react with an oxidant and generate the flame. This complex combustion process is not suitable for being analyzed with a single $1 \mathrm{D}$ reactiondiffusion-advection equation.

\section{DISTRIBUTED-DELAY MODELS}

The models in Sec. II assume that all particles or individuals have the same rest time between two subsequent jumps. In general, this is not the case. Therefore, several authors have developed general reaction-dispersal models including a distribution of dispersal delay times. Such models are usually referred to as reactive continuous-time random walks (CTRWs). Vlad and Ross were the first to apply such models to a specific example, namely a gamma distribution of rest times [17]. Other examples can be found in Refs. [18,19,22]. Here we present a model that provides explicit analytical results for the effective delay time and the front speed $[20,21]$.

\section{A. General model with a waiting-time distribution}

The following model generalizes those in Sec. II to several possible delays [20]. Let $d s P(x, y, t)$ stand for the number of particles per unit area that reach an area $d s$ centered at $(x, y)$ at time $t$. Obviously the number density of particles (or individuals) is given by those that have reached the point considered and still not left,

$$
p(x, y, t)=\int_{0}^{t} d t^{\prime} P\left(x, y, t^{\prime}\right) \Psi\left(t-t^{\prime}\right)
$$

where $\Psi\left(t-t^{\prime}\right)$ is the probability that any particle rests for at least a time interval $t-t^{\prime}$ before performing the next jump,

$$
\Psi\left(t-t^{\prime}\right)=\int_{t-t^{\prime}}^{\infty} d T \varphi(T)=1-\int_{0}^{t-t^{\prime}} d T \varphi(T)
$$

and the evolution equation for $P(x, y, t)$ is clearly

$$
\begin{aligned}
P(x, y, t)= & \int_{0}^{t} d T \int_{-\infty}^{\infty} d \Delta x \int_{-\infty}^{\infty} d \Delta y \\
& P(x-\Delta x, y-\Delta y, t-T) \varphi(T) \phi(\Delta x, \Delta y) \\
& +\rho_{0} \delta(x=0) \delta(y=0) \delta(t=0)+F(x, y, t),
\end{aligned}
$$

where $F(x, y, t)$ is the net number of particles (or individuals) appearing per unit time due to chemical reactions (or biological reproduction).

Fourier-Laplace transforming these three equations and using the definitions (226)-(228), we come to

$\widehat{p}\left(k_{x}, k_{y}, s\right)\left[1-\widehat{\phi}\left(k_{x}, k_{y}\right) \widehat{\varphi}(s)\right]=\frac{1-\widehat{\varphi}(s)}{s}\left[p_{0}+\widehat{F}\left(k_{x}, k_{y}, s\right)\right]$.

Now, in contrast to Sec. II.C, we do not assume a Dirac delta for the distribution of rest times. Instead, we consider an arbitrary distribution $\varphi(T)$ and proceed as follows.

(i) For simplicity, we assume that the space kernel is isotropic, i.e. $\phi(-\Delta x, \Delta y)=\phi(\Delta x, \Delta y)=$ 
$\phi(\Delta x,-\Delta y)=\phi(\Delta y, \Delta x)$, which using the normalization of probability $\left(\int_{-\infty}^{\infty} d \Delta x \int_{-\infty}^{\infty} d \Delta y \quad \phi(\Delta x, \Delta y)=1\right)$ leads to

$$
\begin{aligned}
\widehat{\phi}\left(k_{x}, k_{y}\right) \equiv & \int_{-\infty}^{\infty} d \Delta x \int_{-\infty}^{\infty} d \Delta y e^{-i k_{x} \Delta x-i k_{y} \Delta y} \phi(\Delta x, \Delta y) \\
= & \int_{-\infty}^{\infty} d \Delta x \int_{-\infty}^{\infty} d \Delta y[1-i \vec{k} \cdot \vec{\Delta} \\
& \left.-k_{x}^{2} \frac{\Delta x^{2}}{2}-k_{y}^{2} \frac{\Delta y^{2}}{2}+O\left(\vec{\Delta}^{3}\right)\right] \phi(\Delta x, \Delta y) \\
= & 1-\frac{\left\langle\Delta^{2}\right\rangle}{4}\left(k_{x}^{2}+k_{y}^{2}\right)+O\left(\vec{\Delta}^{3}\right),
\end{aligned}
$$

where $O\left(\vec{\Delta}^{3}\right)$ stands for terms of third and higher powers of $\Delta x$ and $\Delta y$. This approximation will be valid assuming that the dispersal kernel $\phi(\Delta x, \Delta y)$ is appreciably different from zero only for sufficiently small jumps ( $\Delta x \simeq 0, \Delta y \simeq 0$ ). Otherwise, the second-order or 'diffusion' approximation above would break down (leading to what is called long-range dispersal in Ecology [95-97]).

(ii) Analogously to Eq. (125), we assume that the waiting-time probability distribution $\varphi(T)$ is appreciably different from zero only for sufficiently small values of the waiting time $T$, so that we can use again a second-order Taylor expansion,

$$
\begin{aligned}
\widehat{\varphi}(s) & \equiv \int_{0}^{\infty} d T e^{-s T} \varphi(T) \\
& =\int_{0}^{\infty} d T\left[1-s T+s^{2} \frac{T^{2}}{2}+O\left(T^{3}\right)\right] \varphi(T) \\
& =1-s\langle T\rangle+\frac{s^{2}}{2}\left\langle T^{2}\right\rangle+O\left(T^{3}\right)
\end{aligned}
$$

Combining the three previous equations up to second order yields

$$
\begin{aligned}
& \frac{\widetilde{T} s}{2}\left(s \widehat{p}-p_{0}\right)+s \widehat{p}-p_{0}= \\
& -\widetilde{D}\left(k_{x}^{2}+k_{y}^{2}\right) \widehat{p}+\widehat{F}\left(k_{x}, k_{y}, s\right)+\frac{\widetilde{T}}{2} \widehat{F}\left(k_{x}, k_{y}, s\right),
\end{aligned}
$$

where we have defined the reduced diffusion coefficient

$$
\widetilde{D}=\frac{\left\langle\Delta^{2}\right\rangle}{4\langle T\rangle}
$$

and

$$
\begin{aligned}
\widetilde{T} & \equiv 2\langle T\rangle-\frac{\left\langle T^{2}\right\rangle}{\langle T\rangle} \\
& =2 \int_{0}^{\infty} d T \varphi(T) T-\frac{\int_{0}^{\infty} d T \varphi(T) T^{2}}{\int_{0}^{\infty} d T \varphi(T) T} .
\end{aligned}
$$

Antitransformation of Eq. (127) yields an hyperbolic reaction-diffusion (HRD) equation, namely

$$
\begin{aligned}
\frac{\widetilde{T}}{2} \frac{\partial^{2} p}{\partial t^{2}}+\frac{\partial p}{\partial t}= & D\left(\frac{\partial^{2} p}{\partial x^{2}}+\frac{\partial^{2} p}{\partial y^{2}}\right) \\
& +F(x, y, t)+\frac{\widetilde{T}}{2} \frac{\partial F(x, y, t)}{\partial t}
\end{aligned}
$$

In this way, we reach the very interesting result that reaction-diffusion systems can be described by an HRD equation (20), not only for a single value of the waiting time (Sec. II), but also for any general waiting-time distribution $\varphi(T)[21]$. According to Eq. (18), the speed of front solutions to Eq. (130) is

$$
c=\frac{2 \sqrt{r_{L} D}}{1+r_{L} \widetilde{T} / 2} .
$$

Finally, $\widetilde{T}$ defined by Eq. (129) is an effective delay time, first introduced in Ref. [20], and its meaning can be understood by rewriting Eq. (129) as

$$
\widetilde{T}=\langle T\rangle(1-\varepsilon)
$$

where

$$
\varepsilon \equiv \frac{\left\langle(T-\langle T\rangle)^{2}\right\rangle}{\langle T\rangle^{2}}=\frac{\left\langle T^{2}\right\rangle-\langle T\rangle^{2}}{\langle T\rangle^{2}}
$$

is the dispersion of the waiting-time distribution. Physically, we can understand the fact that $\widetilde{T} \neq\langle T\rangle$, i.e. the presence of the last term in Eq. (132), as follows. Consider two waiting time distributions with the same mean $\langle T\rangle$ but different dispersion $\varepsilon$ (Fig. 10). If the distribution shape is wide (Fig. 10.a), some individuals will have low values of the dispersive delay $T$, as compared to the narrower distribution (Fig. 10.b). Intuitively, it is obvious that a population front will travel faster if some individuals move sooner, i.e. with a lower delay $T$ (as in Fig. 10.a as compared to Fig. 10.b). This is the physical reason why the effective delay $\widetilde{T}$ (which tends to slow the front down, see Eq. (131)) will be lower for Fig. 10.a. Thus, $\widetilde{T}$ decreases with increasing values of the dispersion $\varepsilon$, as predicted by Eq. (132). The distributions depicted are discrete, but the argument applies equally to continuous distributions.

It has been shown that Eq. (132) breaks down for $\varepsilon>1$ because then a second-order Taylor expansions are not sufficient and additional terms should be included (see Ref. [20] for details).

We conclude that, for any two waiting-time distributions with the same mean delay time $\langle T\rangle$, for that with higher dispersion $\varepsilon$ some particles jump sooner (lower value of $\widetilde{T}$ ) and make the front move faster (higher value of $c$ ). Conceptually, this effect is somehow similar to long-range dispersal in Ecology [95-97]. There, a few seeds dispersing at further distances can lead to a much faster front. Here, a few particles dispersing sooner can also lead to a faster front. 


\section{B. Discrete delays. Application to the Neolithic transition}

The transition from hunter-gatherer (Paleolithic) into agricultural (Neolithic) economics is a very important process in human history. In Eurasia, it took place in the period 13,000 to 5,000 years Before Present, in the form of a range expansion of farming populations from the Near East [98]. This process can be followed in space and time by using the archaeological record [99]. In this section we review this application of reaction-diffusion fronts.

\section{Fisher's model}

The first quantitative model of the Neolithic transition came after Cavalli-Sforza noted that Fisher's model (24) could be applied to this process. Then the front speed is predicted by Eq. (23) as

$$
c_{\text {Fisher }}=2 \sqrt{r_{L} D},
$$

Ammerman and Cavalli-Sforza [98] gathered the archaeological data available and used them to estimate a observed speed of about $1 \mathrm{~km} / \mathrm{yr}$ (via linear regression). They also estimated the diffusion coefficient as $D \approx \frac{\left\langle\Delta^{2}\right\rangle}{T}$, and found anthropological observations in the literature from which they obtained the characteristic values $r_{L}=0.032 \mathrm{yr}^{-1},\left\langle\Delta^{2}\right\rangle=1544 \mathrm{~km}^{2}$ and $T \approx 25 \mathrm{yr}$. Then Fisher's speed (134) yields $c \approx 2.8 \mathrm{~km} / \mathrm{yr}$. This prediction is much faster than the observed speed that they obtained from the archaeological record, namely $1.0 \pm 0.2$ $\mathrm{km} / \mathrm{yr}[98,4]$.

The two-dimensional formula (19) yields the more accurate estimation $D=\frac{\left\langle\Delta^{2}\right\rangle}{4 T}=386 \mathrm{~km}^{2} /$ gen $=15.44$ $\mathrm{km}^{2} / \mathrm{yr}$, leading to $c_{\text {Fisher }}=1.4 \mathrm{~km} / \mathrm{yr}$ [4], but this is still outside the observed range.

\section{Single-delayed model}

A more refined approach [4] noted that Fisher's equation (24) does not take into account the fact that newborn children spend some time $T$ with their parents until they become adults and can migrate. Therefore, it is reasonable to use the HRD equation (20) instead of Fisher's, and the speed is given by Eq. 18)

$$
c_{\text {single delay }}=\frac{2 \sqrt{r_{L} D}}{1+r_{L} \frac{T}{2}} .
$$

Using the same parameter values as above, this yields a speed of $c_{\text {single delay }}=1.0 \mathrm{~km} / \mathrm{yr}$, which is consistent with the observed range.

\section{Multidelayed model}

More recently, Cavalli-Sforza suggested that a single value for the rest time may not be a realistic description, because in real populations not all children will leave their parents' at the same age. In fact, this was the original motivation to develop the general theory of distributed delays presented in the previous subsection [20]. According to Eq. (131),

$$
c_{\text {multidelay }}=\frac{2 \sqrt{r_{L} \widetilde{D}}}{1+r_{L} \widetilde{T} / 2},
$$

where $\widetilde{D}$ and $\widetilde{T}$ should be estimated from Eqs. (128) and (129), respectively.

A careful examination of the original demographic observations of pre-industrial farmers [100] showed that the observed rest time distribution is $T_{1}=27 \mathrm{yr}, p_{1}=0.46$; $T_{2}=35.5 \mathrm{yr}, p_{2}=0.51 ; T_{3}=45.5 \mathrm{yr}, p_{3}=0.02 ;$ $T_{4}=55.5 \mathrm{yr}, p_{4}=0.01[101]$. Then

$$
\langle T\rangle=32.0 \mathrm{yr}
$$

Eqs. (128), (129) and (136) yield $\widetilde{D}=386 \mathrm{~km}^{2} / \mathrm{gen}=$ $12.06 \mathrm{~km}^{2} / \mathrm{yr}, \widetilde{T}=31.1 \mathrm{yr}$ and $c_{\text {multidelay }}=0.8 \mathrm{~km} / \mathrm{yr}$, respectively (using, as above, $\left\langle\Delta^{2}\right\rangle=1544 \mathrm{~km}^{2}$ and $r_{L}=$ $\left.0.032 \mathrm{yr}^{-1}\right)$. This implies a correction of $17 \%$ relative to model (ii) above, so that this effect should not be neglected a priori.

The detailed analysis above of the demographic data is also interesting, because the estimation $T \approx 25$ yr [98] (used in models (i) and (ii) above) is essentially the mean age at which individuals leave their parents [101], but the multidelayed model (iii) shows that the relevant quantities are the mean time difference between the parents' and the children migration (averaged over all children), $\langle T\rangle$, and the effective delay time, $\widetilde{T}$, given by Eq. (129).

To what extent does the prediction (136) of the multidelayed model depend on the uncertainties in the values of the parameters? In Fig. 11 (continuous curves) we see that, for many values of $r_{L}$ and $\left\langle\Delta^{2}\right\rangle /\langle T\rangle$ in the range allowed by independent observations (hatched rectangle), the predictions of the multi-delayed model are consistent with the observed speed (0.8-1.2 km/yr from the observations quoted above [98,4]; for additional data see Ref. [99]). It is also seen in Fig. 11 that the corrections relative to the single-delayed model are large, about $20 \%$ (essentially because the reevaluation above of the demographic dispersal data shows that the efective delay $\widetilde{T} \simeq 31 \mathrm{yr}$ is higher than the value $T=25 \mathrm{yr}$ used in the single-delay model [4]).

This discrete multi-delayed model is also applicable to physical [26,103] and biological systems [104,105] such that observations imply several possible, discrete values of the rest time in the random walks of particles or individuals. 


\section{Continuous delays. Application to virus infections}

In the previous subsection, we have considered a discrete set of possible waiting times (between subsequent jumps) because the data available for that application were recorded as a discrete set. However, in many cases the measured distribution of waiting times is continuous. For example, in Fig. 12 we reproduce the so-called one-step growth of the virus $\mathrm{T} 7$ infecting E. Coli bacteria. This experiment refers to an homogeneous medium of cells infected at $t=0$. If all viruses took exactly the same time to kill a cell and reproduce, Fig. 12 would be a step function, and the waiting time distribution would be a Dirac delta. Instead, the gradual rise in the virus concentration in Fig. 12 indicates that it takes a different time for each virus to kill the cell it has infected and reproduce, and that a continuous distribution of waiting times is appropriate for this application. This interpretation of one-step curves is well-known in virology [106]. For the case in Fig. 12, we see that the range of waiting times (i.e., the rise in the curve) is between 14 and 23 minutes approximately, so the width of the rise is about 7 minutes, i.e. almost $40 \%$ of the mean value of 18.4 minutes. This is not negligible, so the distribution of waiting times should not be neglected a priori. This subsection reviews the waiting-time distribution for this system and its front speed [21].

As mentioned above, the experimental data in Fig. 12 were obtained for an homogeneous medium of cells infected at $t=0$. Then, since each virus disappears and gives rise to a progeny (or yield) of $Y$ viruses after a time $T$ with probability $\varphi(T)$, obviously the concentration of viruses will in that experiment evolve according to

$$
V=V_{t=0}+\int_{0}^{t} d T \varphi(T)(Y-1) V_{t=0},
$$

so that the waiting-time probability distribution can be obtained from the curve in Fig. 12 as

$$
\varphi(T)=\frac{1}{V_{t=0}(Y-1)} \frac{d V}{d t} .
$$

The inset in Fig. 12 shows the time derivative of the main Fig. 12 (full curve), and a Gaussian fitted by leastsquares (dotted curve). It is seen that a Gaussian is a very good description of the waiting-time distribution of these viruses. Therefore, here we will use a Gaussian waiting-time distribution,

$$
\varphi(T)=\left\{\begin{array}{l}
A \exp \left[-\left(\frac{T-\langle T\rangle}{B}\right)^{2}\right] \quad \text { if } \quad T \geq 0, \\
0 \quad \text { if } \quad T<0,
\end{array}\right.
$$

so that the normalization constant (i.e., the value of $A$ such that $\left.\int_{0}^{\infty} d T \varphi(T)=1\right)$ and the mean squared waiting time $\left\langle T^{2}\right\rangle$ are, respectively,

$$
\begin{gathered}
A=\frac{2}{B \sqrt{\pi}\left(1+\operatorname{Erf}\left[\frac{\langle T\rangle}{B}\right]\right)} \\
\left\langle T^{2}\right\rangle=\int_{0}^{\infty} d T \varphi(T) T^{2}=\frac{B^{2}}{2}+\langle T\rangle^{2} \\
+\frac{B}{\sqrt{\pi}} \frac{\langle T\rangle}{1+\operatorname{Erf}\left[\frac{\langle T\rangle}{B}\right]} \exp \left[-\frac{\langle T\rangle^{2}}{B^{2}}\right],
\end{gathered}
$$

where $\operatorname{Erf}[z] \equiv \frac{2}{\sqrt{\pi}} \int_{0}^{\infty} \exp \left[-t^{2}\right] d t$ is the error function.

The former results become much simpler if we consider the special case that all viruses have a waiting time substantially different from zero. In other words, if we consider the case in which the time between the arrival of a virus and the departure of its progeny is not negligible for any of the viruses (below we shall see that this is indeed realistic). Intuitively, we may express this condition by means of the mathematical inequality

$$
\begin{aligned}
\varphi(T & =0)=A \exp \left[-\left(\frac{\langle T\rangle}{B}\right)^{2}\right] \\
& \ll \varphi_{\max }=\varphi(T=\langle T\rangle)=A,
\end{aligned}
$$

or

$$
\exp \left[-\left(\frac{\langle T\rangle}{B}\right)^{2}\right] \ll 1
$$

In this special case, the first line in Eq. (140) is approximately zero for $T<0$, and we may approximate the normalization condition as follows

$$
1=\int_{0}^{\infty} d T \varphi(T) \simeq \int_{-\infty}^{\infty} d T \quad A \exp \left[-\left(\frac{T-\langle T\rangle}{B}\right)^{2}\right]
$$

which yields

$$
A \simeq \frac{1}{B \sqrt{\pi}}
$$

and Eq. (142) becomes the very simple expression

$$
\begin{aligned}
\left\langle T^{2}\right\rangle & \simeq \int_{-\infty}^{\infty} d T \quad A \exp \left[-\left(\frac{T-\langle T\rangle}{B}\right)^{2}\right] \\
& =\frac{B^{2}}{2}+\langle T\rangle^{2}
\end{aligned}
$$

which below we shall see that is realistic and very useful.

The Gaussian curve fitted to Eq. (140) is shown as a dotted curve in the inset in Fig. 12. It has the parameter values $\langle T\rangle=18.38 \mathrm{~min}$ and $B=1.634 \mathrm{~min}$. Using these values into Eq. (142) yields $\left\langle T^{2}\right\rangle=339.1 \mathrm{~min}^{2}$. The same result can be found from the approximation (147) [because for these values $\exp \left[-\left(\frac{\langle T\rangle}{B}\right)^{2}\right] \sim 10^{-55}$, 
so that the condition (144) holds]. Then, using Eq. (129) we can estimate the effective waiting time, $\widetilde{T}=18.31$ $\min$.

For virus infections the diffusion coefficient $D$ must be replaced by an effective one to take into account the presence of bacteria (which hinder virus diffusion, given by Fricke's equation [107]

$$
D_{e f f}=\frac{1-f}{1+\frac{f}{x}} D
$$

where $x$ takes proper care of the bacterial shape (for $E$. Coli $x \simeq 1.67$ [5]) and $f=B_{0} / B_{\max }$ is the concentration of bacteria relative to its maximum possible value ( $B_{0}$ is the initial bacteria concentration far from the inoculation origin, and it depends on the initial nutrient concentration).

The uninfected bacteria number density $B(x, y, t)$ and infected bacteria density $I(x, y, t)$ evolve according to the following well-known equations

$$
\begin{gathered}
\frac{\partial B}{\partial t}=-k_{1} V B \\
\frac{\partial I}{\partial t}=k_{1} V B-k_{2} I\left(1-\frac{I}{I_{\max }}\right),
\end{gathered}
$$

where $k_{1}$ is the rate constant of the virus adsorption reaction $[V+B \rightarrow I], k_{2}$ the rate constant of the infected bacteria lysis reaction $[I \rightarrow Y \cdot V]$ and $I_{\max }$ the saturation density of infected cells, so the reaction kinetics for viruses is

$$
F(x, y, t)=-k_{1} V B+Y k_{2} I\left(1-\frac{I}{I_{\max }}\right) .
$$

Therefore, using the HRD Eq. (130) we have a system of three simultaneous partial differential equations,

$$
\begin{aligned}
V_{t}+\frac{\widetilde{T}}{2} V_{t t}= & D_{e f f} V_{r r}-k_{1}\left\{V B+\frac{\widetilde{T}}{2}(V B)_{t}\right\}+ \\
& Y k_{2}\left\{I\left(1-\frac{I}{I_{\max }}\right)+\frac{\widetilde{T}}{2}\left[I\left(1-\frac{I}{I_{\max }}\right)\right]_{t}\right\} \\
B_{t}= & -k_{1} V B \\
I_{t}= & k_{1} V B-k_{2} I\left(1-\frac{I}{I_{\max }}\right),
\end{aligned}
$$

which generalize those considered in Ref. [5], where a single delay (i.e., a Dirac delta distribution for $\varphi(T)$ ) was considered.

The solution obtained by linearization in the front frame $z \equiv r-c t \rightarrow \infty,(V, B, I)=\left(\varepsilon_{V}, \varepsilon_{B}, \varepsilon_{I}\right)=$ $\overrightarrow{\varepsilon_{0}} \exp [-\lambda z] \simeq\left(0, B_{0}, 0\right)$, is obtained by requiring the determinant of the matrix corresponding to the linearized form of Eqs. (152) to vanish. This yields

$$
\begin{aligned}
0= & \left(-1+\frac{\widetilde{\widetilde{T}}}{2} \bar{c}^{2}\right) \bar{c} \lambda^{3}+\left(-1+\left(1+\frac{\widetilde{\widetilde{T}}}{2}\left(\kappa_{1}+1\right)\right) \bar{c}^{2}\right) \lambda^{2} \\
& +\left(\kappa_{1} \bar{c}\left(1-\frac{\widetilde{T}}{2}(Y-1)\right)+\bar{c}\right) \lambda-\kappa_{1}(Y-1), \quad(153)
\end{aligned}
$$

where $\kappa_{1} \equiv k_{1} B_{0} / k_{2}$ and $\widetilde{\widetilde{T}} \equiv \widetilde{T} k_{2}$ are dimensionless parameters. This equation can be solved numerically in order to find out the dimensionless front speed $\bar{c} \equiv c / \sqrt{D_{\text {eff }} k_{2}}$ such that $\bar{c}=\min _{\lambda>0}[\bar{c}(\lambda)]$, where $\bar{c}(\lambda)$ is given by characteristic equation (153). Using the rest of the parameter values from the literature [5] and solving Eq. (153) numerically gives the virus front speed the predictions shown in Fig. 13 (curves), which agree well with the experimental data from Refs. [108,70,109] (symbols). No free or adjustable parameters have been used.

From Fig. 13 it is clear that physical models can explain the virus front experiments, contrary to the widespread misbelief that they are driven by unknown biological factors [109]. Very recently, the conditions under which the shape of $\varphi(T)$ has an important effect on the front speeds have been analyzed in detail [21]. Also, approximate explicit formulae for the front speed have been recently derived [39].

Future research topics on this area could include (i) computing virus concentration profiles, and (ii) analyzing the transient after which a mutation induces a change in the front speed. For both processes, there are experimental data available and models similar to that presented here could be applied.

We stress that the general framework in Sec. VI.A can be applied to any waiting-time distribution function.

\section{Cancer tumors and anomalous transport}

Fedotov and Iomin have recently applied exponential and anomalous waiting-time distributions to model cancerous growth [44]. Experimental evidence shows the lower proliferation rate of migratory cells. This is modelled by means of two mutually exclusive cell states, one of them corresponding to migration (state 1) and the other one to proliferation (state 2). Fedotov and Iomin assume that a cell remains in state 1 during a waiting time $T_{1}$ and then switches to state 2 . After a waiting time $T_{2}$, it switches again to state 1 . They derive front speeds in one-dimensional (1D) space for two kinds of waiting-time distributions.

(i) Exponential waiting-time distributions

$$
\varphi\left(T_{i}\right)=\beta_{i} \exp \left(-\beta_{i} T_{i}\right)
$$

where $\beta_{i}$ is the switching rate from state $i$ into the other state. Then the evolution equation of the migratory cells (with density $n_{1}$ ) is 


$$
\frac{\partial n_{1}}{\partial t}=D \frac{\partial^{2} n_{1}}{\partial x^{2}}-\beta_{1} n_{1}+\beta_{2} n_{2}
$$

where $n_{2}$ is the density of cells in the proliferation state.

(ii) The case in which the function $\Psi(t)$, defined by Eq. (122), is of the form

$$
\Psi(t) \sim t^{-1-\gamma}(0<\gamma<1)
$$

This second case corresponds to anomalous transport (diverging mean waiting time $\langle T\rangle$ ), leading to fractional derivatives for the temporal operator, and it slows down of the cancer spreading rate [44]. Anomalous reactiondiffusion equations are also discussed in Ref. [26] for power-law waiting-time distributions $\varphi(T)$.

This is a field of research to which physical models can make in the future additional interesting contributions, e.g. (i) extending such 1D approaches to more dimensions, and (ii) determining the parameter values from independent observations to perform quantitative predictions for the front speed.

Anomalous transport can also arise in the form of divergent second-order moments $\left\langle\Delta^{2}\right\rangle$ from a power-law dispersion kernel (Lévy flights), leading to fractional spatial derivatives [27]. Such kernels are not physically realistic (because in real systems arbitrarily large jumps are always truncated). But fractional derivatives arising from such kernels can provide a realistic description during a transient time, before the effect of the truncation becomes important [103]. Similarly, a waitingtime distribution must be always truncated in real systems (because arbitrarily long waiting times are not possible). Therefore, fractional temporal derivatives arising from such distributions may again be useful in the description of transient effects. Finally, very recently dynamics compatible with fractional temporal derivatives have been experimentally observed in the long-time regime by analyzing the paths of migrating cells [110]. However, it must be stressed that such anomalous diffusion effects (e.g., the mean-squared displacement $\left\langle r^{2}(t)\right\rangle$ not being proportional to time) do not necessarily imply infinite, non-physical values for some moments $(\langle T\rangle$, $\left\langle\Delta^{2}\right\rangle$, etc.) neither fractional-derivative equations (because they are also predicted by other kinds of evolution equations [111]).

\section{SEQUENTIAL MODELS}

\section{A. Temporal order of reproduction and dispersal}

As surveyed in the previous sections, a lot of work has been done based on models derived from the general evolution equation (1). Such models are appropriate for many physical systems (e.g., combustion flames) as well as for biological species such that their dispersion and reproduction are simultaneous and independent. However, this is not the case in some important biophysical phenomena. For example, consider the dispersal of seeds from trees. Such dispersal takes place during a specific period of the year only (e.g., fall), always immediately after reproduction (seed production). Then, dispersal and reproduction are clearly neither simultaneous nor independent. For this reason, the following time-ordered (or sequential) evolution equation for the adult tree number density must be used [112,113], instead of Eq. (1),

$$
\begin{gathered}
p(x, y, t+T)=R_{0} \int_{-\infty}^{+\infty} \int_{-\infty}^{+\infty} p\left(x-\triangle_{x}, y-\triangle_{y}, t\right) \\
\phi\left(\triangle_{x}, \triangle_{y}\right) d \triangle_{x} d \triangle_{y},
\end{gathered}
$$

where $R_{0}$ is the net reproductive rate (number of seeds per parent tree and year which survive into an adult tree) and $T$ is the generation time. Note that the difference with nonsequential models is that the reproduction rate appears multiplicatively in Eq. (157) rather than additively as in the nonsequential Eq. (1).

Another way to introduce this important equation is depicted in Fig. 14. Equation (157) is more realistic than (1) because, as shown in Fig. 14(b), according to (157) seeds (empty circles) are dispersed away from their parent tree (full circle), which does not move. In contrast, as Fig. 14( $a$ ) shows, non-sequential models based on Eq. (1) assume that (i) trees move away and (ii) seeds stay at the original location of their parent tree.

Equation (157) and Fig. $14(b)$ are exact only for species with non-overlapping generations (i.e., such that parent trees reproduce only once and then die) $[112,113]$. But previous results in one-dimensional (1D) space show that substantially more complicated models (with overlapping generations) do not change the order of magnitude of the front speed $[114,115]$, so the approximate Eq. (157) has been applied in recent work $[12,25,11,35]$.

It has been noted that a macroscopic derivation of sequential models (analogous to Sec. II.B for nonsequential models) does not seem possible [12]. The reason is that physical macroscopic equations (Sec. II.B and Fig. 14(a)) do not take into account the fact that in biological populations, it is usually the new generation of individuals that disperses away (Fig. 14(b)). Indeed, this is the main feature of sequential models (Eq. (157)).

Appendix B contains an age-structured derivation of the sequential evolution Eq. (157).

The front speed problem for Eq. (157) is well-known in $1 \mathrm{D}$ space [112-114]. In subsections B-C below, we shall review some recent results in $2 \mathrm{D}$ space and a specific $2 \mathrm{D}$ application [11].

\section{B. Application to Reid's paradox}

Consider an isotropic kernel, i.e. such that $\phi\left(\triangle_{x}, \triangle_{y}\right)$ depends only on

$$
\triangle \equiv \sqrt{\triangle_{x}^{2}+\triangle_{y}^{2}}
$$


Obviously the normalization of probability requires that

$$
\int_{-\infty}^{\infty} \int_{-\infty}^{\infty} d \Delta_{x} d \Delta_{y} \phi(\Delta)=2 \pi \int_{0}^{\infty} d \Delta \Delta \phi(\Delta)=1
$$

and this dispersal probability per unit area $\phi(\Delta)$ (i.e. into a rectangular area $d \Delta_{x} d \Delta_{y}$ ) is related to that per unit length $\varphi(\Delta)$ (i.e. into a $2 \mathrm{D}$ ring of area $2 \pi \Delta d \Delta$ ) as

$$
\varphi(\Delta)=2 \pi \Delta \phi(\Delta),
$$

with the normalization condition

$$
\int_{0}^{\infty} d \Delta \varphi(\triangle)=1
$$

This is useful because the measured or computed kernels are usually $\varphi(\Delta)$ rather than $\phi(\Delta)$ [116].

Bimodal kernels have two components,

$$
\varphi(\triangle)=p_{L} \varphi_{L}(\triangle)+p_{S} \varphi_{S}(\triangle),
$$

with $p_{L}$ the probability of long-distance dispersal (LDD) and $p_{S}$ that of short-distance dispersal, and $\varphi_{i}(\triangle)$ the corresponding kernels $(i=L, S)$. It has been long suspected that such kernels (with characteristic distances differing by several orders of magnitude) may explain a very important, unsolved biophysical problem, namely the fact that the observed speeds of forest postglacial recolonization fronts are much faster than those predicted by single-kernel reaction-dispersal models. This disagreement is called Reid's paradox [114]. Many authors have shown that hypothetical LDD events could solve Reid's paradox [114] using kernels fitted to short-distance data and purely hypothetical LDD events [114,97] (and almost always using 1D models). However, Nathan and coworkers derived and tested very interesting kernels with two components: a short-distance component $\varphi_{S}(\triangle)$ (of the order of 10 meters) and a second, very rare, LDD component $\varphi_{L}(\triangle)$ (covering distances of $10^{3}-10^{4} \mathrm{~m}$, but observed for only about $0.2 \%$ of seeds released from the parent tree, so $\left.p_{L}=1-p_{S} \ll p_{S}\right)$. They derived such bimodal kernels by means of fluid dynamics simulations of atmospheric transport including turbulent-uplifting events that had been previously neglected. They also checked their new kernels by comparing predicted vertical deposition patterns and uplifting probabilities to observed data [116-118]. This opened the possibility to explain Reid's paradox using kernels which are not hypothetical, but derived from physical principles. Recently, front speed formulae for such complex kernels have been derived and tested by $2 \mathrm{D}$ simulations of reactive random walks on grids, showing that the predicted front speeds are about $10^{2}-10^{3} \mathrm{~m} / \mathrm{yr}$ (which are two orders of magnitude higher than those obtained neglecting the LDD component, i.e. for $\left.\varphi_{S}(\triangle)\right)$ [11]. This possible solution to Reid's paradox is reviewed below.

\section{Continuous-space random walk (CSRW) model}

As in section II.C, assume that for $t \rightarrow \infty$ the front is approximately planar at scales much larger than that of individual dispersal events, so that we can choose the $x$-axis parallel to the local velocity of the front. Let $c \equiv\left|c_{x}\right|$ stand for this speed $\left(c_{y}=0\right.$ in the local frame just introduced). We look for constant-shape solutions with the form $p=p_{0} \exp [-\lambda(x-c t)]$ as $x-c t \rightarrow \infty$ and, again, assume that the minimum speed is the one of the front (we will check this assumption by means of numerical simulations in Fig. 15). Then Eq. (157) leads to the asymptotic $(t \rightarrow \infty)$ speed of $2 \mathrm{D}$ fronts

$$
c=\min _{\lambda>0} \frac{\ln \left[R_{0} \widehat{\varphi}(\lambda)\right]}{\lambda T}
$$

where the minimization is relative to $\lambda$,

$$
\widehat{\varphi}(\lambda) \equiv \int_{0}^{\infty} d \Delta \varphi(\Delta) I_{0}(\lambda \Delta),
$$

and

$$
I_{0}(\lambda \Delta) \equiv \frac{1}{2 \pi} \int_{0}^{2 \pi} d \theta \exp [\lambda \Delta \cos \theta]
$$

is the modified Bessel function of the first kind and order zero. The kernel per unit length $\varphi(\Delta)$ is related to that per unit area $\phi(\Delta)$ by Eq. (160).

It is worth to mention that a lot of work on Reid's paradox [114,97] has applied the corresponding $1 \mathrm{D}$ result instead of Eqs. (163)-(165). In fact, it is easy to see that in 1D an equation similar to (163) holds but Eqs. (164)-(165) do not. Thus, the speed $c$ is different in 1D than in 2D. Indeed, it has been shown recently that the $2 \mathrm{D}$ speed is always slower than in $1 \mathrm{D}$ one for the same kernel $\varphi(\triangle)$ provided that it is isotropic [11]. Since in this review we are interested in Reid's paradox, which refers to forest range expansions that took place in 2D, we will focus on the $2 \mathrm{D}$ case here (a comparison to $1 \mathrm{D}$ speeds will be included only in Fig. 15).

\section{Reactive random-walk simulations on grids}

Now we are not dealing with a differential equation, such as Eq. (1), but with an integrodifference equation in 2D, Eq. (157). Therefore, in sharp contrast to Sec. $\mathrm{V}$ (combustion flames) and previous work [1], numerical simulations here cannot use finite-step approximations to derivatives. Thus, recent work $[11,12,25]$ uses simulations that may be called of random-walk or molecular dynamics type (or cellular automata, in the continuous limit for the possible values of $p(x, y, t))$. Such simulations are performed on a 2D grid, with nearest neighbors separated by a distance $D$. Initially $p(x, y, 0)=1$ at $(x, y)=(0,0)$ and 0 elsewhere. At each time step, the new number density of trees $p(x, y, t+T)$ at all nodes of 
the $2 \mathrm{D}$ grid is computed as follows. In agreement with Eq. (157), the seed production $R_{0} p(x, y, t)$ at every node is computed [119] and then redistributed among all grid nodes using the kernel $\phi(\Delta)$. Such $2 \mathrm{D}$ simulations have been performed [11] for values of $R_{0}$ and $T$ typical of the yellow poplar (Liriodendron tulipifera) [120], because the long- and short-distance kernel components $\left[\varphi_{S}(\Delta)\right.$ and $\varphi_{L}(\Delta)$, respectively] of this tree species were determined $[116]$.

Consider first a very simple, short-distance unimodal kernel $\varphi_{S}(\Delta)$ such that is approximately constant for dispersal distances $\Delta<15 \mathrm{~m}$ and zero for $\Delta>15 \mathrm{~m}$ [116]. Using a $2 \mathrm{D}$ grid with nearest neighbors separated by a distance $D=1 \mathrm{~m}$, the simulations agree with the CSRW, as shown in Fig. 15 [121]. This shows (i) the validity of the minimum-speed conjecture [122], and (ii) the need to take Eq. (160) into account in the simulations [123]. But $\varphi_{S}(\Delta)$ is a unimodal kernel. For bimodal ones the time required for the simulations is prohibitively long [124]. Therefore, molecular dynamics simulations are not practical to test the 2D analytical result (163) for bimodal kernels. In the next section we review a fast, efficient approach to test whether Eq. (163) holds or not for bimodal kernels [11].

\section{Discrete-space random walk (DSRW) model}

This model is not exact. But it is necessary to check the CSRW model for bimodal kernels. The DSRW is closely analogous to the numerical simulations, in the following sense. Both in the DSRW and the simulations, the $2 \mathrm{D}$ continuous space is replaced by a grid of points (nodes) with nearest neighbors separated by a distance $D$ along the $x$ and $y$ axes. The nodes are the only points available for seeds and trees. First consider the very simple, highly-idealized case in which any tree disperses seeds only to its 8 nearest neighbors on the grid. Obviously, these 8 final dispersal nodes lie on a square with side $2 D$ and center at the parent tree, as follows. The closest 4 nodes are a distance $\pm D$ away along the $x$ or $y$ directions, and the next 4 are at distance $\pm D$ away along both directions, i.e. on the vertices of the square (at distance $\sqrt{D^{2}+D^{2}}=D \sqrt{2}$ from the parent tree). Then Eq. (157) becomes simply

$$
\begin{aligned}
p(x, y, t+T)= & R_{0}\left\{\frac{P(D)}{4}[p(x-D, y, t)\right. \\
& +p(x+D, y, t) \\
& +p(x, y-D, t) \\
& +p(x, y+D, t)] \\
& +\frac{P(D \sqrt{2})}{4}[p(x-D, y-D, t) \\
& +p(x-D, y+D, t) \\
& +p(x+D, y-D, t) \\
& +p(x+D, y+D, t)]\},
\end{aligned}
$$

where the first 4 terms correspond to horizontal and vertical 'jumps', whereas the last 4 terms are due to diagonal jumps, and the jump probabilities are, from Eq. (160),

$$
P\left(\Delta_{i}\right)=\frac{\phi\left(\Delta_{i}\right)}{\sum_{j=1}^{n} \phi\left(\Delta_{j}\right)}=\frac{\varphi\left(\Delta_{i}\right) /\left(2 \pi \Delta_{i}\right)}{\sum_{j} \varphi\left(\Delta_{j}\right) /\left(2 \pi \Delta_{j}\right)} .
$$

For the simple case of Eq. (166), $n=2$ and the only possible dispersal distances are $\Delta_{1}=D$ and $\Delta_{2}=D \sqrt{2}$.

To derive the speed, we use again the same approach as that applied to the CSRW model above, but now to the DSRW Eq. (166). This yields

$$
c=\min _{\lambda>0} \frac{\ln \left[R_{0}\left(P(D) \frac{[\cosh (\lambda D)+1]}{2}+P(D \sqrt{2}) \cosh (\lambda D)\right)\right]}{\lambda T} .
$$

Note that Eq. (168) is a very simple approximation (DSRW) but is completely analogous to the exact (CSRW) speed (163). It has been found (e.g., for $\varphi_{S}(\Delta)$ above) that this extremely simple DSRW yields a speed (168) which disagrees with that from the CSRW [11]. Therefore, consider dispersal to nodes not on a single, but on many squares $(j=1,2,3 \ldots)$ centered at each parent tree. A square with side $2 j D$ will obviously have $8 j$ nodes, namely 4 at distance $j D, 4$ at distance $j D \sqrt{2}$, and also (except for the simple case $j=1$ above) 8 nodes at distance $\sqrt{(j D)^{2}+(i D)^{2}}$ for $i=1,2, \ldots, j-1$. Finally, in order to use the measured kernels [116] we need to restrict dispersal to a maximum distance in whatever direction, $r_{\max }$. Then it is not difficult to write the analogue to Eq. (166) for bimodal kernels (162) and see that the speed (168) is generalized into

$$
\begin{aligned}
c= & \min _{\lambda>0} \frac{1}{\lambda T} \ln \left[R _ { 0 } p _ { L } \sum _ { j = 0 } ^ { N _ { L } } \left(P_{L}\left(j D_{L}\right)\right.\right. \\
& \frac{\left[\cosh \left(\lambda j D_{L}\right)+1\right]}{2}+P_{L}\left(j D_{L} \sqrt{2}\right) \cosh \left(\lambda j D_{L}\right)+\sum_{i=1}^{j-1} \\
& {\left.\left[P_{L}\left(\sqrt{\left(j D_{L}\right)^{2}+\left(i D_{L}\right)^{2}}\right) \frac{\cosh \left(\lambda i D_{L}\right)+\cosh \left(\lambda j D_{L}\right)}{2}\right]\right) } \\
& +p_{S} \sum_{j=0}^{N_{S}}\left(P_{S}\left(j D_{S}\right)\right. \\
& \frac{\left[\cosh \left(\lambda j D_{S}\right)+1\right]}{2}+P_{S}\left(j D_{S} \sqrt{2}\right) \cosh \left(\lambda j D_{S}\right)+\sum_{i=1}^{j-1} \\
& {\left.\left[P_{S}\left(\sqrt{\left(j D_{S}\right)^{2}+\left(i D_{S}\right)^{2}}\right) \frac{\cosh \left(\lambda i D_{S}\right)+\cosh \left(\lambda j D_{S}\right)}{2}\right]\right) }
\end{aligned}
$$

where $N_{L}=r_{\max L} / D_{L}, N_{S}=r_{\max S} / D_{S}$, and the terms with $\sqrt{\left(j D_{S}\right)^{2}+\left(i D_{S}\right)^{2}}$ arise from jumps in directions different from $0^{\circ}, \pm 45^{\circ}, 180^{\circ}$ and $\pm 90^{\circ}$. The probabilities are related by Eq. (167) to the corresponding dispersion kernel, for example 


$$
\begin{aligned}
& P_{L}\left(j D_{L}\right)=\frac{\varphi_{L}\left(j D_{L}\right)}{2 \pi j D_{L}} / \\
& \sum_{j}^{N_{L}}\left[\frac{\varphi_{L}\left(j D_{L}\right)}{2 \pi j D_{L}}+\frac{\varphi_{L}\left(j D_{L} \sqrt{2}\right)}{2 \pi j D_{L} \sqrt{2}}+\sum_{i=1}^{j-1} \frac{\varphi_{L}\left(\sqrt{\left(j D_{S}\right)^{2}+\left(i D_{S}\right)^{2}}\right)}{2 \pi \sqrt{\left(j D_{S}\right)^{2}+\left(i D_{S}\right)^{2}}}\right] .
\end{aligned}
$$

For the yellow poplar (Liriodendron tulipifera), the LDD component of the kernel derived (and favorably compared to observations) in Refs. [116-118] can be fitted to a curve of the form

$$
\varphi_{L}(\Delta)=\left\{\begin{array}{l}
0 \\
10^{3.903} \Delta^{-2.301}<10^{3} \mathrm{~m} \\
0 \quad \Delta>10^{4} \mathrm{~m}, 10^{3} \mathrm{~m} \leq \Delta \leq 10^{4} \mathrm{~m}
\end{array}\right.
$$

so $r_{\max L}=10^{4} \mathrm{~m}$, whereas, as mentioned above, its short-distance component $\varphi_{S}(\Delta)$ can be taken as approximately constant for $\Delta<15 \mathrm{~m}$ and zero for $\Delta>15 \mathrm{~m}$, so $r_{\max S}=15 \mathrm{~m}$.

\section{Propagation speeds}

Figure 16 presents the results for the bimodal kernel (162), where $p_{L}=0.00202$ and $p_{S}=1-p_{L}$ are the probabilities of long-distance and short-distance dispersal (obtained from Ref. [116]). The results for the unimodal kernels $\varphi_{L}(r)$ and $\varphi_{S}(r)$ are also presented for comparison.

Both the 2D DSRW and the 2D CSRW models show conclusively that the front speeds for the bimodal kernels (162), using the parameter values derived in [116], are about $10^{2}-10^{3} \mathrm{~m} / \mathrm{yr}$, i.e. two orders of magnitude faster than those for the unimodal, short-range component $\varphi_{S}(r)$ (Fig. 16). Speeds of $10^{2}-10^{3} \mathrm{~m} / \mathrm{yr}$ are, in fact, those required to solve Reid's paradox [114].

Note from the upper curve in Fig. 16 (100\% of seeds with $\left.\operatorname{LDD}, \varphi_{L}\right)$ that the same order of magnitude $\left(10^{2}\right.$ $\mathrm{m} / \mathrm{yr}$ ) is obtained as with only $0.2 \%$ of seeds with LDD (middle curve).

It is worth to stress that short-distance kernels $\varphi_{S}(r)$ have been measured experimentally many times. But bimodal kernels with a long-distance dispersal (LDD) component $\varphi_{L}(r)$ were derived by a mechanistic (or physical) model for the first time in Ref. [116].

We close this section with the conclusion [11] that Reid's paradox of rapid forest range expansions can be solved (as far as the order of magnitude is concerned), by taking into account the bimodal dispersal kernels derived and favorably compared to data in Refs. [116-118].

Similar results (and detailed comparisons between predicted speeds in 2D and 1D) have been very recently obtained for a list of tree species such that their invasion spread rates have been measured from the paleorecord (using reproductive rates and dispersal kernels appropriate to each species). The predicted rates are again of similar magnitude to the measured ones [35].

\section{COHABITATION MODELS}

\section{A. Special features of human populations}

In section II we have introduced non-sequential models (see Fig. 14(a) and Eq. (1)). They are useful in many physical and biophysical systems. But in Sec. VII we have reviewed the fact that such models are known to be inappropriate in some cases (e.g. for tree species). Then sequential models can be applied [97,112-114] (see Fig. 14(b) and Eq. (157)). This crucial point shows the importance of taking into account biological factors when applying physical models to systems composed of living organisms.

In some cases further considerations are necessary. For example, consider human populations. Non-sequential models based on Eq. (1) have a drawback because they would imply that newborn children (empty circles in Fig. 14(a)) stay at their birth location, whereas their parents (full circles) migrate away from them. A more realistic framework is provided by sequential models based on Eq. (157), because according to them migrating parents live at their final location with their newborn children (Fig. $14(b))$. But below we will see that a more detailed discussion is necessary before applying Eq. (157) to human populations.

As explained in Sec. VII.A, the non-overlapping generations model (Fig. 14(b)) does not take into account the yearly reproduction and dispersal of seeds: all trees reproduced only once and then die. Also, the time step is one generation, and it is the same for all trees (e.g. $T=20$ yr for the yellow poplar, from the previous section). Similarly we can consider (again as an approximation) a model in which all humans take the same time interval to reproduce (e.g. $T=32 \mathrm{yr}$, from Sec. VI.B). But there is a fundamental difference between trees and humans. Trees produce seeds at the location of the parent tree, and the parent tree cannot move. In contrast, humans can have children not only before migration, but also after or during it (Fig. 17). Mathematically, we have the following three main cases.

(a) Migration before reproduction (Fig. 17(a)). The evolution equation is

$$
\begin{aligned}
p(x, y, t+T)= & \int_{-\infty}^{+\infty} \int_{-\infty}^{+\infty} p\left(x-\triangle_{x}, y-\triangle_{y}, t\right) \\
& \phi\left(\triangle_{x}, \triangle_{y}\right) d \triangle_{x} d \triangle_{y} \\
& +R\left[\int_{-\infty}^{+\infty} \int_{-\infty}^{+\infty} p\left(x-\triangle_{x}, y-\triangle_{y}, t\right)\right. \\
& \left.\phi\left(\triangle_{x}, \triangle_{y}\right) d \triangle_{x} d \triangle_{y}\right]
\end{aligned}
$$

with $R[\ldots]$ an appropriate reproduction function (as discussed below). The first term in the RHS corresponds to the parents (black circles in Fig. 17(a)) and the last one to their children (empty circles). Note that the integral within the parentheses [...] gives the population density at the arrival location. 
(b) Reproduction before migration. Then the appropriate equation is (see Fig. 17(b))

$$
\begin{aligned}
p(x, y, t+T)= & \int_{-\infty}^{+\infty} \int_{-\infty}^{+\infty} p\left(x-\triangle_{x}, y-\triangle_{y}, t\right) \\
& \phi\left(\triangle_{x}, \triangle_{y}\right) d \triangle_{x} d \triangle_{y} \\
& +\int_{-\infty}^{+\infty} \int_{-\infty}^{+\infty} R\left[p\left(x-\triangle_{x}, y-\triangle_{y}, t\right)\right] \\
& \phi\left(\triangle_{x}, \triangle_{y}\right) d \triangle_{x} d \triangle_{y} .
\end{aligned}
$$

Now within the parentheses [...] the population density at the origin location appears (instead of an integral).

(c) Reproduction during migration. None of the former two equations applies in general. Instead, according to Fig. 17(c) [125]

$$
\begin{aligned}
p(x, y, t+T)= & \int_{-\infty}^{+\infty} \int_{-\infty}^{+\infty} p\left(x-\triangle_{x}, y-\triangle_{y}, t\right) \\
& \phi\left(\triangle_{x}, \triangle_{y}\right) d \triangle_{x} d \triangle_{y} \\
& +\int_{-\infty}^{+\infty} \int_{-\infty}^{+\infty} R\left[p\left(x^{\prime}, y^{\prime}, t^{\prime}\right)\right] \\
& \phi\left(\triangle_{x}, \triangle_{y}\right) d \triangle_{x} d \triangle_{y} .
\end{aligned}
$$

Consider the following reproduction function [16],

$$
R[p(x, y, t)]=\left\{\begin{array}{ll}
\left(R_{0}-1\right) p(x, y, t) & \text { if } p<p_{\max } \\
0 & \text { if } p \geq p_{\max }
\end{array} .\right.
$$

If the net reproduction rate $R_{0}$ is assumed to be independent of the jump length vector $\left(\triangle_{x}, \triangle_{y}\right)$, position $(x, y)$ and time $t$, then at the leading edge of the front $\left(p \ll p_{\max }\right)$ all three cases yield the same linearized equation, namely

$$
\begin{gathered}
p(x, y, t+T)=R_{0} \int_{-\infty}^{+\infty} \int_{-\infty}^{+\infty} p\left(x-\triangle_{x}, y-\triangle_{y}, t\right) \\
\phi\left(\triangle_{x}, \triangle_{y}\right) d \triangle_{x} d \triangle_{y},
\end{gathered}
$$

so the front speed will be the same for the three cases above [126].

Obviously, Eq. (176) is nothing but Eq. (157). Therefore, although some species (e.g., humans) have special features relative to those considered in the previous section (e.g., trees), the former careful analysis shows that it is still reasonable to apply Eq. (157) if the net reproduction rate $R_{0}$ is constant [16]. But in the case of human populations it seems clearer to refer to Eq. (176) as a cohabitation model, rather than a sequential one (because unlike trees, for humans the time order of reproduction and dispersal is not fixed, see Fig. 17). The term cohabitation refers to the fact that newborn children have to spend some time with their parents until they can survive on their own, a behavior that is not captured by classical models (Fig. 14(a) and Secs. II-III). Below we review some recent work on this topic $[12,16,25]$.

\section{B. Cohabitation reaction-diffusion (CRD) fronts}

Performing Taylor expansions up to second order in space and time, Eq. (176) becomes

$$
\begin{aligned}
\frac{1-R_{0}}{T} p+p_{t}+\frac{T}{2} p_{t t}= & R_{0}\left(-U_{x} p_{x}-U_{y} p_{y}\right. \\
& \left.+U_{x y} P_{x y}+D_{x} p_{x x}+D_{y} p_{y y}\right)
\end{aligned}
$$

where $U_{x}, U_{y}, D_{x}, D_{y}$ and $U_{x y}$ are given by Eqs. (4)-(6). Equation (177) is a cohabitation analogue to Eq. (2).

As in Sec. II.C, we look for solutions with the form $p=p_{0} \exp [-\lambda(x-c t)]$ as $x-c t \rightarrow \infty$, with $c>0$ and $\lambda>0$. Then Eq. (177) yields the characteristic equation

$$
\lambda^{2}\left(D_{x} R_{0}-\frac{T c^{2}}{2}\right)+\lambda\left(-c+U_{x} R_{0}\right)+\frac{R_{0}-1}{T}=0 .
$$

Solving this equation for $\lambda$ and requiring for it to be real, we obtain the condition

$$
\begin{aligned}
g(c) \equiv & c^{2}\left(1+2\left(R_{0}-1\right)\right)-2 c U_{x} R_{0} \\
& +U_{x}^{2} R_{0}^{2}-4 R_{0} \frac{R_{0}-1}{T} D_{x} \geq 0 .
\end{aligned}
$$

It is easily seen that $g(c)$ is convex from below, and that the equation $g(c)=0$ has one negative and one positive root for $c$, say $c_{-}$and $c_{+}$. Therefore, the minimum possible value for $c>0$ corresponds to $c_{+}$, and we finally obtain the speed

$$
c=\frac{R_{0} U_{x}+\sqrt{R_{0}\left(R_{0}-1\right)\left[\frac{4}{T}\left(2 R_{0}-1\right) D_{x}-2 R_{0} U_{x}\right]}}{\left(2 R_{0}-1\right)} .
$$

For the special case of a non-biased random walk, this becomes

$$
\lim _{U_{x} \rightarrow 0} c_{0}^{c}=\sqrt{\frac{4 R_{0} D}{T} \frac{R_{0}-1}{2 R_{0}-1}},
$$

where we have introduced $D \equiv D_{x}$.

In order to compare to the non-cohabitation model in Secs. II-III, it is necessary to establish the connection between the low-density population number growth parameters, namely $r_{L}$ (see Eq. (12)) in classical models and $R_{0}$ (see Eq. (176)) in cohabitation models. This relationship can be obtained most easily as follows. In the absence of dispersal, the classical model becomes the logistic equation (237). For low values of $p(x, y, t)$, it yields

$$
p(x, y, t+T)=p(x, y, t) \exp \left[r_{L} T\right]
$$

whereas, also in the absence of dispersal, $\phi\left(\triangle_{x}, \triangle_{y}\right)$ is a Dirac delta centered at the origin and the cohabitation Eq. (176) becomes 


$$
p(x, y, t+T)=R_{0} p(x, y, t),
$$

so that the reproduction function (175) and the logistic (12) are consistent with each other at low values of the population density, provided that

$$
r_{L}=\frac{1}{T} \ln R_{0}
$$

On the other hand, the reproduction function (175) and the logistic (12) will give different results for high values of the population density $p(x, y, t)$. However, the high-density behavior is not accurately known for biological populations outside the laboratory, because there are no experimentally well-established trends in the population numbers versus time (except at low population densities) [53]. Moreover, the high- $p$ behavior of reproduction does not affect the speed of fronts, as is obvious from both the sequential speed (180) and the non-cohabitation one (17).

Although a comparison to the non-cohabitation model does not seem possible for an arbitrary bias $U_{x}$ [127], it is possible in the non-biased limit $\left(U_{x}=0\right)$. For this purpose, using Eq. (184) into (181) it is easily seen that the speed from the cohabitation or sequential model (181) will be higher than that from the classical model, Eq. (18), provided that

$$
\exp [\tau](\exp [\tau]-1)(1+\tau / 2)^{2}-\tau(2 \exp [\tau]-1)>0
$$

where $\tau \equiv \operatorname{Tr}_{L}>0$. Plotting the left-hand-side for $\tau>0$, it is easily seen that this condition is always fulfilled. The physical interpretation is that the classical model corresponds to simultaneous dispersal and reproduction (Fig. $14(a))$. Intuitively, this should clearly lead to slower invasion fronts that the sequential or cohabitation model (Fig. 14(b)). This is the physical interpretation of the fact that cohabitation models lead to faster front speeds than classical ones.

\section{European invasion of North America in the XIX century}

In Fig. 18 we present the speeds predicted by the noncohabitation model (Eq. (17), lower curve, also shown in Fig. 1) and the cohabitation model (Eq. (180), upper curve) as a function of the random walk bias $\beta$, see Eq. (33).

In Fig. 18, the non-cohabitation model seems compatible with the observed speed for high enough values of $\beta$, whereas the cohabitation model seems not. In principle, we expected the cohabitation model to perform better than the classical model for this application (because it involves a biological population). But from Fig. 18, it appears that it does not [12]. However, this may be too strong a conclusion in view of the uncertainty of the values of the parameters. We think that dispersion data in many directions should be analyzed in order to estimate the mobility $\left(D_{x}\right)$ and bias $\left(U_{x}\right)$ parameters for this human population accurately, as well as their error ranges and their dependence on position. This would yield a nonhomogeneous framework which, in contrast to that in Ref. [60], would be free of some relatively strong assumptions (e.g., the fractal nature of pathways, the use of adjustable parameters, etc.). Such a project would certainly require very tedious work and discussions, which we feel more appropriate for a specialized demography publication. In this review, our aim is not to present an in-depth analysis of the demographic data. Rather, the main point is to show that cohabitation models can be useful to describe such kind of biophysical processes. Indeed, they yield quite different speeds than classical models (Fig. 18). We also think that this is an important lesson showing that physical models cannot be applied straightforwardly to systems of living individuals without taking proper care of their biological features (e.g., sequential reproduction and dispersion for trees; cohabitation of non-adults with adults for humans; etc.).

\section{The Neolithic transition in Europe}

Here we summarize some recent work on cohabitation models beyond the CRD (or second-order) approximation [Eqs. (177)-(181)], and their application to the Neolithic transition [16].

\section{Continuous-space random walk (CSRW) model}

As stressed above, the final cohabitation equation (176) is the same as the sequential one (157). Therefore, Eqs. (163)-(165) can be applied. In order to perform the integrals in Eqs. (163)-(165), an expression for the kernel $\varphi(\triangle)$ is necessary. There are many possible choices of the kernel. For the purposes of this subsection, it will be clearer to assume simply that an individual will either remain at rest (with probability $p_{e}$, which is called the persistence in demography) or move a distance $r$ (with probability $1-p_{e}$ ),

$$
\varphi(\triangle)=p_{e} \delta^{(1)}(\Delta)+\left(1-p_{e}\right) \delta^{(1)}(\Delta-r),
$$

where $\delta^{(1)}(\Delta-r)$ is the 1-dimensional Dirac delta centered at $\Delta=r$. Hence, this simple model assumes that all moving individuals travel the same distance $r$. This makes it possible to find relatively simple analytical results and to run relatively fast random-walk simulations.

Performing the integrals in Eqs. (163)-(165), an explicit expression for the speed is obtained,

$$
c=\min _{\lambda>0} \frac{\ln \left[R_{0}\left(p_{e}+\left(1-p_{e}\right) I_{0}(\lambda r)\right)\right]}{T \lambda}
$$


where the minimization is relative to $\lambda, I_{0}(\lambda r)$ is the modified Bessel function of the first kind and order zero, given by Eq. (165).

Figure 19 shows the speed predicted by the CSRW (full line), Eq. (187), for the following values of $T, R_{0}, p_{e}$ and $r$ typical of human Neolithic populations. The generation time is $T=1$ generation= $32 \mathrm{yr}$ (see Eq. (137)). The net reproductive rate $R_{0}$ can be estimated from population numbers versus time for two human populations that settled in previously unpopulated areas [128]. What is impressive of those data is that, when plotted against the elapsed time in generations, both datasets yield almost exactly the same curve of population number $P(t)$ (divided by its initial value) versus time. From those data and Eq. (176) integrated over the area available, we can estimate the value of $R_{0}=P(t+T) / P(t)$ for several values of $t=T, 2 T, 3 T \ldots$ This yields an average of $R_{0}=2.2$. The lowest value is $R_{0}=1.9$, and the highest one is $R_{0}=2.6$. Thus, in Fig. 19 we compute front speeds for values of $R_{0}$ in the range $1.6-3.0$. On the other hand, the population persistence $p_{e}$ (i.e., the fraction of the population that does not move appreciably) can be estimated directly from the mobility data in Ref. [100], p. 139, for three different populations of preindustrial agriculturalists. The corresponding values of $p_{e}$ are $0.54,0.40$ and 0.19 . In Fig. 19, we use the mean value $p_{e}=0.38$ (the dependence of the front speed of $p_{e}$ shall be analyzed later, in Fig. 21). Finally, the value of $r$ is estimated directly from that of the persistence and the mean-squared displacement [129].

In Fig. 19, the front speed predicted by the CSRW (full line), Eq. (187), is seen to increase with increasing values of the population net reproductive rate $R_{0}$, as expected intuitively. Before analyzing the implications for the Neolithic transition and comparing to previous work, we first review the use of numerical simulations in order to check the validity of the result (187), which in turn relies on several assumptions (as mentioned above).

\section{Reactive random-walk simulations on grids}

We consider a $2 \mathrm{D}$ grid with $10^{3} \cdot 10^{3}$ nodes and initially $p(x, y, 0)=1$ at the central node and 0 elsewhere. At each time step (corresponding to $T=1$ generation), we compute the new population number density $p(x, y, t+T)$ at all nodes of the $2 \mathrm{D}$ grid as follows.

(i) First we compute the new local population density due to reproduction at every node as $R_{0} p(x, y, t)$ if this result is lower than $p_{\max }$ (Eq. (176)) and $p_{\max }$ otherwise (second line in Eq. (175)).

(ii) Then we redistribute this result among all grid nodes using the kernel (186), i.e. we consider that a fraction $p_{e}$ of the population stays at the original node, and the remaining fraction is distributed equally among the nearest neighbors, i.e. a fraction $\left(1-p_{e}\right) / 4$ jumps a distance $\pm r$ along each horizontal or vertical direction.
In the horizontal/vertical directions, the speed obtained from the simulations (circles in Fig. 19) is up to $5 \%$ higher than that predicted by the CSRW (full line). But if we measure the speed along a diagonal $\left(45^{\circ}\right)$ direction (squares in Fig. 19), it is lower than the CSRW. The average of both results (triangles) agrees within about $2 \%$ with the analytical result from the CSRW (full line) [130]. Why does the speed from the random-walk simulations depend on the direction? Because unlike the CSRW, they are not isotropic (motion is only allowed in the horizontal and vertical directions). As shown in Fig. 20, after two generations (dashed arrows), the dispersal distance along the diagonal direction $(r \sqrt{2})$ is lower than that in the horizontal direction $(2 r)$. This is the intuitive reason why the simulation speeds in the diagonal directions (squares in Fig. 20) are lower than in the horizontal/vertical directions (circles in Fig. 20). An analytical approach to this problem is reviewed in the next subsection.

\section{Discrete-space random walks (DSRWs)}

Here we summarize a discrete-space model (similar to that in Sec. VII.B.3 but for the kernel (186)) that is useful in order to test direction-dependent speeds observed in the simulations above [16].

First choose the $\mathrm{X}$ and $\mathrm{Y}$ axes shown in Fig. 20. Then, for the kernel (186), particles can jump into point $(x, y)$ from points $(x \pm r, y)$ and $(x, y \pm r)$. Therefore, Eq. (176) becomes

$$
\begin{aligned}
p(x, y, t+T)= & R_{0}\left\{p_{e} p(x, y, t)+\left(1-p_{e}\right)\left[\frac{1}{4} p(x-r, y, t)\right.\right. \\
& +\frac{1}{4} p(x+r, y, t)+\frac{1}{4} p(x, y-r, t) \\
& \left.\left.+\frac{1}{4} p(x, y+r, t)\right]\right\} .
\end{aligned}
$$

As in Sec. II.C, we look for solutions with the form $p=$ $p_{0} \exp [-\lambda(x-c t)]$ and assume that the minimum speed is the one of the front. In this way we obtain the speed

$$
c=\min _{\lambda>0} \frac{\ln \left[R_{0}\left(\frac{p_{e}+1}{2}+\frac{1-p_{e}}{2} \cosh (\lambda r)\right)\right]}{\lambda T},
$$

where the minimization is relative to $\lambda$. This equation has no analytical solution. However, for given values of $R_{0}, p_{e}, r$ and $T$ it is easy to find its minimum numerically. In this way we obtain the x-crosses in Fig. 19. They agree almost perfectly with the horizontal/verticaldirection simulations, performed in the previous section (circles in Fig. 19).

Now we choose X' and Y' axes shown in Fig. 20. Then, for the same kernel, it is easily seen that particles can jump into point $\left(x^{\prime}, y^{\prime}\right)$ from points $\left(x^{\prime} \pm \frac{r}{\sqrt{2}}, y^{\prime} \pm \frac{r}{\sqrt{2}}\right)$. Therefore, instead of Eq. (188) we have 


$$
\begin{aligned}
p\left(x^{\prime}, y^{\prime}, t+T\right)= & R_{0}\left\{p_{e} p\left(x^{\prime}, y^{\prime}, t\right)+\left(1-p_{e}\right)\right. \\
& {\left[\frac{1}{4} p\left(x^{\prime}+\frac{r}{\sqrt{2}}, y^{\prime}+\frac{r}{\sqrt{2}}, t\right)\right.} \\
& +\frac{1}{4} p\left(x^{\prime}+\frac{r}{\sqrt{2}}, y^{\prime}-\frac{r}{\sqrt{2}}, t\right) \\
& +\frac{1}{4} p\left(x^{\prime}-\frac{r}{\sqrt{2}}, y^{\prime}+\frac{r}{\sqrt{2}}, t\right) \\
& \left.\left.+\frac{1}{4} p\left(x^{\prime}-\frac{r}{\sqrt{2}}, y^{\prime}--\frac{r}{\sqrt{2}}, t\right)\right]\right\} .
\end{aligned}
$$

which leads us, in the same way, to the speed

$$
c=\min _{\lambda>0} \frac{\ln \left[R_{0}\left(p_{e}+\left(1-p_{e}\right) \cosh \left(\lambda \frac{r}{\sqrt{2}}\right)\right)\right]}{\lambda T},
$$

instead of (189). This speed, shown as crosses $(+)$ in Fig. 19, agrees perfectly with the diagonal-direction simulations (squares in Fig. 19).

The agreement between the DSRW model and the simulations (Fig. 19) confirms the validity of the simulations on grids reviewed in the previous subsection, as well as the direction-dependence of the front speed on discrete spaces for the kernel (186).

\section{Propagation speed of the Neolithic transition}

The front speed predicted from the second-order noncohabitation (HRD) approximation (20) and logistic population number growth (12) is given by Eq. (18),

$$
c_{H R D}=\frac{2 \sqrt{r_{L} D}}{1+r_{L} T / 2},
$$

where $D$ is given by Eq. (19). The prediction from Eq. (192) is shown in Fig. 19 as a dashed curve (HRD). It is seen that this second-order (HRD) approximation (which was derived and used in Ref. [4]) is not reliable, since its predicted speed is up to $31 \%$ less than that of the CSRW developed and tested above [131]. This shows very clearly the limitations of the HRD equation in Ref. [4], even for the very simple kernel considered (namely, that in which all moving individuals jump the same distance). We stress that we have chosen this kernel because we wanted to review the limitations of the approach in Ref. [4] for a single, the simplest possible case. Clearly, in future work it would be interesting to extend these methods (CSRW, random-walk simulations on grids and DSRWs) to a variety of kernels appropriate for preindustrial agriculturalist societies.

Previous work [4] did not consider explicitly that some individuals or particles can remain at rest. Instead, all information about the dispersal kernel was 'averaged' into a single parameter, namely the diffusion coefficient (19). Therefore, those models did not make it possible to analyze the effect of persistence on the predicted speed [132].
In contrast, the approaches reviewed above make this possible [16]. In Fig. 21, we compare the CSRW and simulation results for a range of values of the persistence consistent with the observed intergenerational mobility data of preindustrial farmers (see Sec. D.1 above).

Originally the speed of the Neolithic transition in Europe was estimated as $0.8-1.2 \mathrm{~km} / \mathrm{yr}$, using a dataset of only 53 archaeological sites [133]. Over the years, a much larger dataset has become available. Very recently, the 95\%-confidence level speed was estimated as 0.6-1.3 $\mathrm{km} / \mathrm{yr}$ using a dataset of 735 sites [99]. The speeds predicted by the cohabitation, more accurate model (full curves in Figs. 19 and 21) are consistent with this observed range.

The reason why the speed increases with increasing values of the persistence $p_{e}$ (Fig. 21) is that, for a given value of the diffusion coefficient (19), a higher value of the persistence implies that some individuals or families move larger distances per generation -so the reaction front moves faster, due to these long-range migration events.

Figure 21 also shows that, over a wide range of persistence values, there is again good agreement between the CSRW model (full curves) and the random-walk simulations (triangles), for three different values of the net reproductive rate $R_{0}$.

Finally, the second-order approximation to the CSRW result is given by Eq. (181),

$$
c_{C R D}=\sqrt{\frac{2 R_{0} D}{T\left(1+\frac{1}{2\left(R_{0}-1\right)}\right)}} .
$$

This may be called the cohabitation reaction-diffusion (CRD) speed, and is included in Figs. 19 and 21 (dasheddotted curves). It is a good approximation to the exact cohabitation speed (CSRW, full curves in Figs. 19 and 21).

As in Fig. 19, we see from Fig. 21 that the HRD approximation (which was used in Ref. [4]) largely underestimates the results from the exact, cohabitation model. Differences between the cohabitation model and the HRD equation [4] are as large as 70\% (see Fig. 21). This percentage is important, and enough to be measurable, because it is twice as large as the uncertainty in the observed speed of the Neolithic front. Cohabitation models are therefore relevant. Moreover, their interest is not restricted to the Neolithic transition, because they can be applied to other human range expansions, biological invasions, the spread of epidemics and plagues, cultural fronts, etc.

\section{E. Several-population models}

Interaction effects between several species or population types lead to important changes in the dynamics of physical, chemical and biophysical systems $[1,98]$. In 
this subsection we review recent work on such effects for integro-difference evolution equations [25]. For definiteness we shall discuss them in the context of the Neolithic transition.

\section{Continuous-space random walk (CSRW) model}

Let $p_{N}(x, y, t)$ stand for the population number density of the Neolithic population, per unit area centered at position $(x, y)$ and time $t$. The dispersal kernel $\phi_{N}\left(\triangle_{x}, \triangle_{y}\right)$ is the probability per unit area that an individual who was at $\left(x-\triangle_{x}, y-\triangle_{y}, t\right)$ jumps to $(x, y, t+T)$ and $T$ is the time interval between two subsequent jumps $(T=1$ generation $\simeq 32$ years, see Sec. VI.B $)$. Let $R_{N}\left[p_{N}(x, y, t)\right]$ stand for the net effect of reproduction (births minus deaths) of the Neolithic population during the time interval $T$. The cohabitation evolution equation (176) is generalized into

$$
\begin{aligned}
p_{N}(x, y, t+T)= & R_{0 N} \int_{-\infty}^{+\infty} \int_{-\infty}^{+\infty} \\
& p_{N}\left(x-\triangle_{x}, y-\triangle_{y}, t\right) \\
& \phi_{N}\left(\triangle_{x}, \triangle_{y}\right) d \triangle_{x} d \triangle_{y} \\
& +\Gamma \int_{-\infty}^{+\infty} \int_{-\infty}^{+\infty} \\
& p_{N}\left(x-\triangle_{x}, y-\triangle_{y}, t\right) \\
& p_{P}\left(x-\triangle_{x}, y-\triangle_{y}, t\right) \\
& \phi_{N}\left(\triangle_{x}, \triangle_{y}\right) d \triangle_{x} d \triangle_{y},
\end{aligned}
$$

where the last term corresponds to the interaction (with strength $\Gamma$ ) of the invading Neolithic humans with the indigenous Paleolithics. The latter have number density $p_{P}(x, y, t)$, driven by an analogous equation,

$$
\begin{aligned}
p_{P}(x, y, t+T)= & R_{0 P} \int_{-\infty}^{+\infty} \int_{-\infty}^{+\infty} \\
& p_{P}\left(x-\triangle_{x}, y-\triangle_{y}, t\right) \\
& \phi_{P}\left(\triangle_{x}, \triangle_{y}\right) d \triangle_{x} d \triangle_{y} \\
& -\Gamma \int_{-\infty}^{+\infty} \int_{-\infty}^{+\infty} \\
& p_{N}\left(x-\triangle_{x}, y-\triangle_{y}, t\right) \\
& p_{P}\left(x-\triangle_{x}, y-\triangle_{y}, t\right) \\
& \phi_{P}\left(\triangle_{x}, \triangle_{y}\right) d \triangle_{x} d \triangle_{y} .
\end{aligned}
$$

The interaction (last term in both equations) leads to an increase in the population density of species $N$ and a decrease in that of $P$ (so we may represent this process as $N+P \rightarrow N+N)$. This effect is observed in anthropology and may be due to a variety of causes, such as interbreeding or acculturation [98]. In any case, (i) parents will not migrate away from their newborn children (cohabitation), so that it is more appropriate to use an integral over the dispersal kernel also for the interaction term; (ii) the number of new $N$ individuals at $\left(x-\triangle_{x}, y-\triangle_{y}, t\right)$ equals the number of $P$ individuals disappearing at the same space-time point, namely $\Gamma p_{N}\left(x-\triangle_{x}, y-\triangle_{y}, t\right)$ $p_{P}\left(x-\triangle_{x}, y-\triangle_{y}, t\right)$.

For later use, we rewrite the previous set as

$$
\begin{aligned}
p_{N}(x, y, t+T)= & R_{0 N} \int_{-\infty}^{+\infty} \int_{-\infty}^{+\infty} \\
& {\left[1+\gamma p_{P}\left(x-\triangle_{x}, y-\triangle_{y}, t\right)\right] } \\
& p_{N}\left(x-\triangle_{x}, y-\triangle_{y}, t\right) \\
& \phi_{N}\left(\triangle_{x}, \triangle_{y}\right) d \triangle_{x} d \triangle_{y}, \\
p_{P}(x, y, t+T)= & R_{0 P} \int_{-\infty}^{+\infty} \int_{-\infty}^{+\infty} \\
& {\left[1-\frac{\gamma R_{0 N}}{R_{0 P}} p_{N}\left(x-\triangle_{x}, y-\triangle_{y}, t\right)\right] } \\
& p_{P}\left(x-\triangle_{x}, y-\triangle_{y}, t\right) \\
& \phi_{P}\left(\triangle_{x}, \triangle_{y}\right) d \triangle_{x} d \triangle_{y},
\end{aligned}
$$

where

$$
\gamma \equiv \frac{\Gamma}{R_{0 N}}
$$

We assume that the invasion front of the population $N$ spreads in a region where the density of the indigenous one $P$ is initially equal to its maximum possible value, $p_{\max } P$. This is appropriate for the Neolithic transition (i.e., the invasion of Neolithic farmers $N$ into a space populated by indigenous Paleolithic hunter-gatherers $P$ ) [98]. Thus, in the leading edge of the invasion front we may write

$$
\begin{aligned}
& p_{N}(x, y, t) \simeq \varepsilon(x, y, t)+O(2), \\
& p_{P}(x, y, t) \simeq p_{\max } P-\delta(x, y, t)+O(2),
\end{aligned}
$$

where $O(2)$ stands for second and higher-order terms,

$$
\varepsilon(x, y, t) \ll p_{\max } N
$$

and

$$
\delta(x, y, t) \ll p_{\max } P .
$$

Therefore, up to first order we have for the interaction term

$$
\gamma p_{N}(x, y, t) p_{P}(x, y, t) \simeq \gamma p_{N}(x, y, t) p_{\max } P+O(2) .
$$

Such an approach has been applied to several sets of evolution equations $[24,25,134]$. It is useful here because it reduces Eq. (196) to an evolution equation in which only the variable $p_{N}(x, y, t)$ appears,

$$
\begin{aligned}
p_{N}(x, y, t+T) \simeq & R_{0 N}\left(1+\gamma p_{\max P}\right) \\
& \int_{-\infty}^{+\infty} \int_{-\infty}^{+\infty} p_{N}\left(x-\triangle_{x}, y-\triangle_{y}, t\right) \\
& \phi_{N}\left(\triangle_{x}, \triangle_{y}\right) d \triangle_{x} d \triangle_{y} .
\end{aligned}
$$


The front speed of the invading species (farmers in the case of the Neolithic transition) can be found most easily as in Sec. II.C, i.e. by assuming that $p_{N}=$ $p_{0} \exp [-\lambda(x-c t)]$ as $x-c t \rightarrow \infty$. For an isotropic kernel $\phi_{N}(\triangle)$, we obtain from Eq. (203)

$$
\begin{aligned}
\exp [c T \lambda]= & R_{0}\left(1+\gamma \quad p_{\max P}\right) \\
& \int_{0}^{\infty} d \Delta \Delta \phi_{N}(\triangle) \int_{0}^{2 \pi} d \theta \exp [-\lambda \Delta \cos \theta]
\end{aligned}
$$

where $\theta \equiv \tan ^{-1} \frac{\triangle_{y}}{\triangle_{x}}$. As in the previous subsection, we are interested in the simplest possible kernel such that we can derive analytical formulae, so we again assume Eq. (186),

$$
\varphi_{N}(\triangle)=\varphi_{P}(\triangle)=p_{e} \delta^{(1)}(\Delta)+\left(1-p_{e}\right) \delta^{(1)}(\Delta-r)
$$

where the kernel per unit length $\varphi(\Delta)$ is related to that per unit area $\phi(\Delta)$ according to Eq. (160). In fact, there are some small differences between the observed dispersal kernels of pre-industrial farmers, $\varphi_{N}(\triangle)$, and hunter-gatherers, $\varphi_{P}(\triangle)$, but these differences are small [37]. Therefore, we assume simply $\phi_{N}(\triangle) \simeq \phi_{P}(\triangle)$ in Eq. (205). This will avoid substantially more complicated simulations and analyses (which we do not expect to change the results appreciably).

After integrating Eq. (204), we assume as usual that the minimum speed is the one of the front (in the next section, we will check this assumption by means of numerical simulations of the two-species system (196)(197)). In this way we obtain the front speed

$$
c=\min _{\lambda>0} \frac{\ln \left[R_{0 N}\left(1+\gamma p_{\max } P\right)\left(p_{e}+\left(1-p_{e}\right) I_{0}(\lambda r)\right)\right]}{T \lambda},
$$

where $I_{0}(\lambda r)$ is the modified Bessel function of the first kind and order zero, given by Eq. (165). For the case in which a single species invades the habitat without interaction $\left(\gamma=0\right.$ or $\left.p_{\max } P=0\right)$, we recover the singlespecies result (187).

In Fig. 22, we show the speed predicted by the CSRW (full line) for parameter values appropriate for the Neolithic transition $\left(T=32 \mathrm{yr}, R_{0 P}=1.8 /\right.$ gen, $p_{e}=0.38$ and $\left.p_{\max } P=0.064 / \mathrm{km}^{2}\right)$ [25]. In Fig. 22, the front speed predicted by the CSRW (full line), Eq. (206), is seen to increase with increasing values of the interaction parameter $\gamma$, as expected intuitively because the higher its value, the more hunter-gatherers become farmers per generation (see, e.g., Eq. (203)).

\section{Cohabitation reaction-diffusion (CRD) approximation}

Equation (206) is not easy to apply in practice because it requires plotting a function and/or finding its minimum numerically for each set of parameter values. Therefore, here we derive a simpler approximation. We approximate Eq. (203) by using Taylor expansions in space and time up to second order (assuming again an isotropic kernel),

$$
\begin{aligned}
p_{N}+T \frac{\partial p_{N}}{\partial t}+\frac{T^{2}}{2} \frac{\partial^{2} p_{N}}{\partial t^{2}} \simeq & R_{0 N}\left(1+\gamma p_{\max P}\right) p_{N} \\
& +R_{0 N}\left(1+\gamma p_{\max P}\right) \\
& D T\left(\frac{\partial^{2} p_{N}}{\partial x^{2}}+\frac{\partial^{2} p_{N}}{\partial y^{2}}\right),
\end{aligned}
$$

where $D$ is given by Eq. (19). The speed of this CRD equation may be derived, again, by assuming solutions with the form

$$
p \simeq p_{0} \exp [-\lambda(x-c t)]
$$

with $\lambda>0$. This yields

$$
\begin{aligned}
\lambda= & \left(T c+\left[(T c)^{2}-4\left(R_{0 N}\left(1+\gamma p_{\max P}\right)-1\right)\right.\right. \\
& \left.\left.\left(R_{0 N}\left(1+\gamma \quad p_{\max P}\right) D T-\frac{T^{2} c^{2}}{2}\right)\right]^{\frac{1}{2}}\right) \\
& /\left(2 R_{0 N}\left(1+\gamma p_{\max P}\right) D T-T^{2} c^{2}\right) .
\end{aligned}
$$

Requiring $\lambda$ to be real and assuming that the minimum speed is that of the front, we obtain the speed

$$
c_{C R D}=\sqrt{\frac{2 R_{0 N}\left(1+\gamma p_{\max P}\right) D}{T\left(1+\frac{1}{2\left(R_{0 N}\left(1+\gamma p_{\max } P\right)-1\right)}\right)}} .
$$

In Fig. 22 we have also included this speed (dotted curves). It is seen to be a useful approximation, and it is much simpler to use than the exact result (206). The approximate result (210) has been also applied to estimate the coexistence time between the invading and the invaded populations [25]. For the special case in which a single species invades the habitat without interaction $\left(\gamma=0\right.$ or $\left.p_{\max } P=0\right)$, we recover the single-species result (193).

\section{Reactive random-walk simulations on grids}

We consider a $2 \mathrm{D}$ lattice with $10^{3} \cdot 10^{3}$ nodes. Initially the invading population $(N)$ is restricted to the central node of the grid (where $p_{N}(x, y, 0)=p_{\max } N$ ), and $p_{N}(x, y, 0)=0$ elsewhere. For the indigenous population $(P)$, initially $p_{P}(x, y, 0)=0$ at the central node and $p_{P}(x, y, 0)=p_{\max } P$ elsewhere.

At each time step (corresponding to $T=1$ generation $=$ $32 \mathrm{yr}$ ), we compute the new population number densities $p_{N}(x, y, t+T)$ and $p_{P}(x, y, t+T)$ at all nodes of the $2 \mathrm{D}$ lattice as follows.

(i) First, according to the factor $\left[1+\gamma p_{P}\right] p_{N}$ in Eq. (196), at every node we add to the $p_{N}$ the term $\gamma p_{N} p_{P}$. 
And according to the factor $\left[1-\frac{\gamma R_{0 N}}{R_{0 P}} p_{N}\right] p_{P}$ in Eq. (197), we subtract to $p_{P}$ the term $\frac{\gamma R_{0 N}}{R_{0 P}} p_{N} p_{P}$, unless a negative value for $p_{P}$ is obtained. In the latter case we set $p_{P}=0$ (local extinction of the invaded population).

(ii) Second, the dispersion of the population densities obtained in step (i) are performed using the kernel (205). Thus, a fraction $p_{e}=0.38$ of each population $(N$ and $P)$ stays at the original node, and the remaining fraction is distributed equally among the nearest neighbors, i.e. a fraction $\left(1-p_{e}\right) / 4$ jumps a distance $\pm r$ along each horizontal or vertical direction. In the analytical model, this corresponds to the integrations in Eqs. (196)-(197).

(iii) Finally, we compute $p_{N}(x, y, t+T)$ by multiplying $p_{N}(x, y, t)$ (obtained from step (ii)) by the factor $R_{0 N}$ (see Eq. (196)), unless a value $p_{N}>p_{\max } N$ is obtained. In the latter case we set $p_{N}=p_{\max } N$ (to avoid biologically unrealistic population densities over the saturation value implied by the environment). Analogously, $p_{P}(x, y, t+T)$ is computed as $R_{0 P}$ times the value of $p_{P}(x, y, t)$ from step (ii) (unless a value $p_{P}>p_{\max } P$ is obtained; in such as case we again set $p_{P}=p_{\max } P$ ).

For the net reproductive rate of hunter-gatherers, we use the characteristic value $R_{0 P}=1.8$ [135]. Saturation population densities for pre-industrial farmers and hunter-gatherers have been measured for several populations. In Fig. 22 we use the same values as those applied by Currat and Excoffier [136] in their genetic simulations of the Neolithic transition, namely $p_{\max } N=1.28$ farmers $/ \mathrm{km}^{2}$ and $p_{\max } P=0.064$ hunter-gatherers $/ \mathrm{km}^{2}$.

We repeat this 3 -step cycle many times, until we observe that the front speed is constant (this happens before 500 cycles or generations).

Along the horizontal/vertical directions of the lattice, the speed obtained from the simulations (circles in Fig. 22 ) is faster that measured along the diagonal directions [ $\pm 45^{\circ}$ relative to the horizontal axis] (squares). This is similar to Fig. 19, and again due to the reason explained in Fig. 20. The average of both speeds from the simulations (triangles in Fig. 22) agrees with the CSRW (full curves). We could try to attain better agreement by computing the simulated speeds along many other directions. Although the validity of the analytical result is clear from Fig. 22 (curves versus triangles), one expects such calculations to further improve the agreement between the model and the simulations.

The small differences are not unexpected after all, because on a continuous surface jumps take place into all infinite points of a circle (CSRW model) but in simulations they necessarily take place into the nodes of a square (i.e., on a discrete surface). This also explains the asymptotic behavior of the diagonal simulations (squares) for $R_{0 N}=3.0$ in Fig. 22 [137]. We check these simulation results analytically in the next subsection.

\section{Discrete-space random walks (DSRWs)}

For a grid in 2D space and the kernel (205), individuals can jump into point $(x, y)$ from points $(x \pm r, y)$ and $(x, y \pm$ $r)$. Therefore, in discrete space Eq. (203) is replaced by

$$
\begin{aligned}
p_{N}(x, y, t+T)= & R_{0 N}\left(1+\gamma \quad p_{\max } P\right) \\
& \left\{p_{e} p_{N}(x, y, t)+\left(1-p_{e}\right)\right. \\
& {\left[\frac{1}{4} p(x-r, y, t)+\frac{1}{4} p(x+r, y, t)\right.} \\
& \left.\left.+\frac{1}{4} p(x, y-r, t)+\frac{1}{4} p(x, y+r, t)\right]\right\} .
\end{aligned}
$$

As in Sec. II.C, we look for solutions with the form $p=$ $p_{0} \exp [-\lambda(x-c t)]$ and assume that the minimum speed is the one of the front. In this way we come to the speed

$$
c=\min _{\lambda>0} \frac{\ln \left[R_{0 N}\left(1+\gamma p_{\max P}\right)\left(p_{e}+\frac{1-p_{e}}{2}[\cosh (\lambda r)+1]\right)\right]}{\lambda T} .
$$

This equation has no analytical solution. However, for given values of $R_{0}, p_{e}, r, T$ and $\gamma$ it is easy to find its minimum numerically. In this way we obtain the $\mathrm{x}$-crosses in Fig. 22. They agree perfectly with the horizontal/vertical-direction random-walk simulations, performed in the previous section (circles in Fig. 22).

Now we choose $X^{\prime}$ and $Y^{\prime}$ forming $45^{\circ}$ with the $\mathrm{X}$ and $\mathrm{Y}$ axes (see Fig. 20). Then, individuals jump into point $\left(x^{\prime}, y^{\prime}\right)$ from points $\left(x^{\prime} \pm \frac{r}{\sqrt{2}}, y^{\prime} \pm \frac{r}{\sqrt{2}}\right)$ so, instead of Eq. (211) we have

$$
\begin{aligned}
p\left(x^{\prime}, y^{\prime}, t+T\right)= & R_{0}\left(1+\gamma p_{\max } P\right) \\
& \left\{p_{e} p\left(x^{\prime}, y^{\prime}, t\right)+\left(1-p_{e}\right)\right. \\
& {\left[\frac{1}{4} p\left(x^{\prime}+\frac{r}{\sqrt{2}}, y^{\prime}+\frac{r}{\sqrt{2}}, t\right)\right.} \\
& +\frac{1}{4} p\left(x^{\prime}+\frac{r}{\sqrt{2}}, y^{\prime}-\frac{r}{\sqrt{2}}, t\right) \\
& +\frac{1}{4} p\left(x^{\prime}-\frac{r}{\sqrt{2}}, y^{\prime}+\frac{r}{\sqrt{2}}, t\right) \\
& \left.\left.+\frac{1}{4} p\left(x^{\prime}-\frac{r}{\sqrt{2}}, y^{\prime}--\frac{r}{\sqrt{2}}, t\right)\right]\right\} .
\end{aligned}
$$

which leads us, in the same way, to the speed

$c=\min _{\lambda>0} \frac{\ln \left[R_{0}\left(1+\gamma p_{\max P}\right)\left(p_{e}+\left(1-p_{e}\right) \cosh \left(\lambda \frac{r}{\sqrt{2}}\right)\right)\right]}{\lambda T}$,

instead of (212). This speed is shown as crosses $(+)$ in Fig. 22. It agrees perfectly with the diagonal-direction simulations (squares in Fig. 22). 


\section{Effect of the interaction on the front propagation speed}

Both the analytical results and the simulations (Fig. 22) are seen to be consistent with the observed speed of the Neolithic transition in Europe, namely $0.6 \leq c \leq 1.3$ $\mathrm{km} / \mathrm{yr}$ [99], provided that the interaction parameter $\gamma$ is low enough, e.g. $\gamma<5 \mathrm{~km}^{2}$ for $R_{0 N}=3.0$. Such a high value for $R_{0 N}$ is usually regarded as the highest possible net reproduction rate for pre-industrial agriculturalists, and it is considered reasonable for Neolithic range expansions [138]. In principle, however, lower values could apply to regions less favorable for agriculture (e.g. $\quad R_{0 N}=1.6$, which is the lowest value consistent with the population number series in Ref. [128], so we also include it in Fig. 22).

The interaction parameter $\gamma$ determines the strength of the interaction between the two species (or populations, in the case of the Neolithic transition). This parameter is important to predict the range expansion speed (Fig. 22). It is also of crucial importance in models of the geographic distribution of genes after a range expansion [136]. Computer simulations of Eqs. (196)-(197) and analytical formulae have recently shown that the values of $\gamma$ used in Fig. 22 are in reasonable agreement with the values of the coexistence time between the Neolithic and Paleolithic populations, as estimated from archaeological observations [25]. This line of research opens the way towards regional analyses in which: (i) observed geographic differences in the coexistence times [136] could be used to estimate non-homogeneous values for the interaction parameter $\gamma$ and therefore for the front speed (Fig. 22); (ii) regions less suitable for agriculture may correspond to lower values for $R_{0 N}$ and thus have a slower front speed (Fig. 22), which is consistent with the empirical observation that the Neolithic front slowed down as it approached colder regions in Northern Europe [139].

\section{CONCLUSIONS AND PERSPECTIVES}

We have presented microscopic, macroscopic and age-structured derivations of reaction-dispersion and reaction-diffusion equations arising from biased random walks, distributed delays, sequential models, dispersive variability, etc. For all cases, we have derived formulae for the speeds of their front solutions. Applications here surveyed include diffusive and convective effects on the front propagation speed of combustion flames, Reid's paradox of rapid forest spread, the colonization of NorthAmerica in the XIX century, the Neolithic transition in Europe, the spread of genetic mutations, subsistence and cultural boundaries, virus infections, cancer tumors and anomalous transport, etc.

Several approaches have been reviewed and compared for some relevant cases, including the use of FourierLaplace transforms for distributed delays, continuousspace and discrete-space random-walk models (CSRW and DSRWs, respectively), reactive random-walk simulations on 2-dimensional grids, etc.

For combustion flames (Sec. V), research results published during the last five years have made it possible to reduce a system of coupled equations to a single equation for a reduced temperature variable, which in turn has made it possible to derive lower and upper bounds on the flame front speed. This framework has been extended to include mass diffusion, convective effects and temperature-dependent transport coefficients (Figs. 5-9).

For distributed delays (Sec. VI), a reduced hyperbolic reaction-diffusion equation (130) and an effective delay time (132) have been derived and applied to the Neolithic transition (Fig. 11) and to virus infections (Figs. 12-13).

Sequential models (Sec. VII) take into account the fact that dispersal and biological reproduction are not simultaneous (Fig. 14). In recent years, work on such models in two-dimensional spaces has provided a possible solution to Reid's paradox of rapid forest range expansion (Figs. 15-16).

Cohabitation models have the same mathematical form as sequential models, but in contrast to the latter, they do not imply any specific order between dispersal and reproduction. They do take into account that newborn humans have to spend some time with their parents until they can survive on their own. This effect is important when computing human population front speeds (Figs. 19 and 21).

The models and formulae here reviewed can be useful in a lot of applications. Many possible lines of future research have been proposed in several sections of this review. Moreover, biased front models can be useful for a variety of physical and biophysical applications dealing with biased fronts, such as particle diffusion in disordered lattices [47], nucleation of spiral waves [14], human and nonhuman population invasions [104], the spread of epidemics [140], cultural fronts [38], etc.

The 2D sequential model of non-overlapping generations (Sec. VII.A) could be extended to allow for overlapping generations, and applied to Reid's paradox (Sec. VII.B).

Another field of future research should be the use of cohabitation models (Sec. VIII) with a variety of dispersal kernels, as appropriate for specific applications.

The two-species model reviewed in the last subsection could be applied to competition systems (in which both species have a detrimental effect on the other one).

An additional, especially augurious field of research on reaction-diffusion fronts is that of microorganisms, because in such systems experiments can be easily replicated and the parameter values are simpler to estimate [5].

We close this review by stressing that for purely physical applications [14,47] (not involving biological reproduction), non-sequential models (Secs. II-V) are more appropriate. For biophysical ones [104,140], distributeddelay (Sec. VI), sequential (Sec. VII) and cohabitation models (Sec. VIII) seem more reasonable (depending on 
the features of the underlying random walks and reproductive processes).

\section{ACKNOWLEDGMENTS}

Funded by the European Commission (grant NEST28192-FEPRE), the MEC-FEDER (grant FIS-200612296-C02-02) and the Generalitat de Catalunya (grant SGR-2005-00087).

\section{APPENDIX A. AGE-STRUCTURED DERIVATION OF NON-SEQUENTIAL MODELS}

Here we present a more detailed derivation of the model in Secs. II.A-B, by taking the possibility of agedependent mortality and natality into account. Using recent results by Vlad [17], the classical approach by Othmer, Dunbar and Alt [141] has been generalized to include reproductive processes which may depend on the age structure of the population [16].

\section{A. Age-structured derivation of Eqs. (1), (3) and logistic growth (12)}

Let $\Sigma(a, x, y, t)$ stand for the number density (per unit area) of individuals aged $a$ that reach an area centered at point $(x, y)$ at time $t$. The total number of individuals $P(x, y, t)$ reaching the same location at $t$ is

$$
P(x, y, t)=\int_{0}^{\infty} d a \Sigma(a, x, y, t) .
$$

Let $\rho(a, x, y, t)$ stand for the number density (per unit area) of individuals aged $a$ at point $(x, y)$ at time $t$. The total number of individuals $p(x, y, t)$ at the same location at $t$ is

$$
p(x, y, t)=\int_{0}^{\infty} d a \rho(a, x, y, t) .
$$

These definitions imply the following evolution equations for $\Sigma(a, x, y, t)$ and $\rho(a, x, y, t)$

$$
\begin{aligned}
& \Sigma(a, x, y, t)=\int_{0}^{t} d T \varphi(T) \int_{-\infty}^{+\infty} \int_{-\infty}^{+\infty} d \triangle_{x} d \triangle_{y} \\
& \Sigma\left(a-T, x-\triangle_{x}, y-\triangle_{y}, t-T\right) \phi\left(\triangle_{x}, \triangle_{y}\right) \\
& +\rho_{0}(a) \delta(x) \delta(y) \delta(t)+\delta(a) \int_{0}^{\infty} d a^{\prime} \lambda\left(a^{\prime}\right) \rho\left(a^{\prime}, x, y, t\right) \\
& -\mu_{0}(a) \rho(a, x, y, t)-\mu_{1} p(x, y, t) \rho(a, x, y, t),
\end{aligned}
$$

$$
\rho(a, x, y, t)=\int_{0}^{t} d t^{\prime} \Sigma\left(a, x, y, t^{\prime}\right) \Psi\left(t-t^{\prime}\right),
$$

where, as in Sec. II.A, the dispersal kernel $\phi\left(\triangle_{x}, \triangle_{y}\right)$ is the probability per unit area that a particle (or individual) who was at $\left(x-\triangle_{x}, y-\triangle_{y}, t\right)$ jumps to $(x, y, t+T)$.
The distribution $\varphi(T)$ is the probability that it rests for a time between $T$ and $T+d T$ before performing the next jump, divided by $d T$. The term $\rho_{0}(a) \delta(x) \delta(y) \delta(t)$ corresponds to assuming that initially the density of individuals aged $a$ is $\rho_{0}(a)$ at the origin, and it vanishes elsewhere. $\lambda(a)$ and $\mu_{0}(a)$ are the aged-dependent natality and mortality rates per individual, respectively. Finally, $\mu_{1}$ is an additional mortality term which avoids an unbounded growth of the population density, as shown below (additional, higher-order terms could be included, but are not necessary for our purposes). Note that, in contrast the death terms (last two terms in Eq. (217)), the birth term is an integral over age $a$ involving the natality rate $\lambda(a)$, because individuals of different ages may reproduce, i.e. contribute to the population with 0 -aged individuals (thus the factor $\delta(a)$ within this term). In Eq. (218) we have introduced the probability that a particle (or individual) rests for at least a time interval $t-t^{\prime}$ before performing the next jump,

$$
\Psi\left(t-t^{\prime}\right)=\int_{t-t^{\prime}}^{\infty} d T \varphi(T)=1-\int_{0}^{t-t^{\prime}} d T \varphi(T),
$$

so that Eq. (218) simply states that the particles at $(x, y, t)$ are those that have arrived at some earlier time and still not left.

Integrating Eq. (218) over age $a$ yields

$$
p(x, y, t)=\int_{0}^{t} d t^{\prime} P\left(x, y, t^{\prime}\right) \Psi\left(t-t^{\prime}\right) .
$$

Vlad $[142,143,17]$ has shown that, after a transient of a few generations, the age structure of the population reaches a stationary distribution $c_{s t}(a)$ which is also uniform in space, i.e.

$$
\rho(a, x, y, t)=p(x, y, t) c_{s t}(a)
$$

The following parameter, introduced by Lotka [144],

$$
r_{L}=\int_{0}^{\infty} d a\left[\lambda(a)-\mu_{0}(a)\right] c_{s t}(a)
$$

is called the intrinsic or initial growth rate of the population number density (the reason of these names will become clear at the end of this Appendix).

Although this is not necessary for the purposes of the present review, Vlad $[142,143,17]$ has also shown that $c_{s t}(a)$ is given by Lotka's distribution [144],

$$
c_{s t}(a)=\frac{e^{-r_{L} a} e^{-\int_{0}^{a} d a^{\prime} \mu_{0}\left(a^{\prime}\right)}}{\int_{0}^{\infty} d a e^{-r_{L} a} e^{-\int_{0}^{a} d a^{\prime} \mu_{0}\left(a^{\prime}\right)}},
$$

so that $r_{L}$ satisfies Lotka's transcendental equation

$$
\int_{0}^{\infty} d a \lambda(a) e^{-r_{L} a} e^{-\int_{0}^{a} d a^{\prime} \mu_{0}\left(a^{\prime}\right)}=1 .
$$

Integration of Eq. (217) over age leads to 


$$
\begin{aligned}
& P(x, y, t)=\int_{0}^{t} d T \varphi(T) \int_{-\infty}^{+\infty} \int_{-\infty}^{+\infty} d \triangle_{x} d \triangle_{y} \\
& P\left(x-\triangle_{x}, y-\triangle_{y}, t-T\right) \phi\left(\triangle_{x}, \triangle_{y}\right) \\
& +p_{0} \delta(x) \delta(y) \delta(t) \\
& +r_{L} p(x, y, t)-\mu_{1} p^{2}(x, y, t)
\end{aligned}
$$

where $p_{0}=\int_{0}^{\infty} d a \rho_{0}(a)$ and we have used Eqs. (221)(222).

In order to solve Eq. (225), we introduce the FourierLaplace transforms of the corresponding space-time fields $[145,146]$

$$
\begin{aligned}
\Sigma_{n=1}^{\infty} \frac{T^{n}}{n !} \frac{\partial^{n} p}{\partial t^{n}}= & \int_{-\infty}^{+\infty} \int_{-\infty}^{+\infty} p\left(x-\triangle_{x}, y-\triangle_{y}, t\right) \\
& \phi\left(\triangle_{x}, \triangle_{y}\right) d \triangle_{x} d \triangle_{y} \\
& -p(x, y, t)+\Sigma_{n=1}^{\infty} \frac{T^{n}}{n !} \frac{\partial^{n-1} F(x, y, t)}{\partial t^{n-1}} .
\end{aligned}
$$

From Eq. (230) we obtained the so-called logistic growth function,

$$
F(x, y, t)=r_{L} p(x, y, t)-\mu_{1} p^{2}(x, y, t) .
$$

, Therefore, we reach the final result

$$
\begin{aligned}
& p(x, y, t+T)-p(x, y, t) \\
& =\int_{-\infty}^{+\infty} \int_{-\infty}^{+\infty} p\left(x-\triangle_{x}, y-\triangle_{y}, t\right) \\
& \phi\left(\triangle_{x}, \triangle_{y}\right) d \triangle_{x} d \triangle_{y} \\
& -p(x, y, t)+\Sigma_{n=1}^{\infty} \frac{T^{n}}{n !} \frac{\partial^{n-1} F[p(x, y, t)]}{\partial t^{n-1}}
\end{aligned}
$$

$\widehat{p}\left(k_{x}, k_{y}, s\right)=\int_{-\infty}^{\infty} d x \int_{-\infty}^{\infty} d y \int_{0}^{\infty} d t e^{-i \vec{k} \cdot \vec{x}-s t} p(x, y, t)$

$$
\begin{aligned}
\widehat{\varphi}(s) \widehat{\phi}\left(k_{x}, k_{y}\right)= & \int_{0}^{\infty} d T e^{-s T} \varphi(T) \int_{-\infty}^{\infty} d \Delta x \\
& \int_{-\infty}^{\infty} d \Delta y e^{-i \vec{k} \cdot \overrightarrow{\Delta x}} \phi(\Delta x, \Delta y) .
\end{aligned}
$$

We now Fourier-Laplace transform the Eq. (225) (see e.g. Ref. [145], formulae (F.5b,k) and (F.11g,n)),

$$
\widehat{P}\left(k_{x}, k_{y}, s\right)=\widehat{P}\left(k_{x}, k_{y}, s\right) \widehat{\phi}\left(k_{x}, k_{y}\right) \widehat{\varphi}(s)+p_{0}+\widehat{F}\left(k_{x}, k_{y}, s\right),
$$

where

$$
\widehat{F}\left(k_{x}, k_{y}, s\right)=r_{L} \widehat{p}\left(k_{x}, k_{y}, s\right)-\mu_{1} \widehat{p^{2}}\left(k_{x}, k_{y}, s\right) .
$$

Second, we Fourier-Laplace transform Eq. (220) (see Ref. [146], formulae $(32.13,25))$,

$$
\widehat{p}\left(k_{x}, k_{y}, s\right)=\widehat{P}\left(k_{x}, k_{y}, s\right) \frac{1-\widehat{\varphi}(s)}{s} .
$$

Combining the two latter equations we get rid of the field $\widehat{P}\left(k_{x}, k_{y}, s\right)$. Assuming a Dirac-delta distribution for $\varphi(T)$, we have

$$
\frac{1}{\widehat{\varphi}(s)}=\exp [s T]=1+\Sigma_{n=1}^{\infty} \frac{T^{n} s^{n}}{n !}
$$

and we obtain

$$
\begin{aligned}
& \sum_{n=1}^{\infty} \frac{T^{n} s^{n-1}}{n !}\left(s \widehat{p}\left(k_{x}, k_{y}, s\right)-p_{0}\right) \\
& =\left[\widehat{\phi}\left(k_{x}, k_{y}\right)-1\right] \widehat{p}\left(k_{x}, k_{y}, s\right) \\
& +\sum_{n=1}^{\infty} \frac{T^{n} s^{n-1}}{n !} \widehat{F}\left(k_{x}, k_{y}, s\right) .
\end{aligned}
$$

Antitransforming this equation yields so that using equation (3), we obtain Eq. (1) with $R[p(x, y, t)]$ given by $(3)$ and $F$ given by (235) or (12). This completes the age-structured derivation of Eq. (1). The advantages of the derivation presented in this subsection are: (i) It shows that the evolution equation (1) is valid for biologically reasonable situations (in the sense that natality and mortality rates may depend on age); (ii) Using Eq. (222), one can compute the effect of agedependent natality and mortality rates on the evolution equation, and thus on the front speed (this latter problem is analyzed in the next subsection $\mathrm{D})$.

\section{B. Special cases}

Note that in the absence of dispersal, the first and second terms in the RHS of Eqs. (234), (236) and (1) do not appear. Then, either Eq. (234) or (1) leads to

$$
\frac{\partial p(x, y, t)}{\partial t}=r_{L} p(x, y, t)-\mu_{1} p^{2}(x, y, t)
$$

The solution of this equation is the well-known logistic growth [52],

$p(x, y, t)=\frac{\frac{r_{L}}{\mu_{1}} p_{0}(x, y)}{p_{0}(x, y)+\left(\frac{r_{L}}{\mu_{1}}-p_{0}(x, y)\right) \exp \left[-r_{L} t\right]} \underset{t \rightarrow \infty}{\rightarrow} \frac{r_{L}}{\mu_{1}} \equiv p_{\max }$,

where $p_{0}(x, y,) \equiv p(x, y, t=0)$.

However, in the special case $\mu_{1}=0$ Eq. (237) is replaced by

$$
\frac{\partial p(x, y, t)}{\partial t}=r_{L} p(x, y, t)
$$

and then the growth of the population (238) becomes exponential, 


$$
p(x, y, t)=p_{0}(x, y) \exp \left[r_{L} t\right] \underset{t \rightarrow \infty}{\rightarrow},
$$

which leads to an unbounded growth of the population. This is not observed in any real biophysical system. A widely-used, simple way to avoid this divergence is just to include the last term $\left(\mu_{1} \neq 0\right)$ in Eq. (217) -so that Eq. (238) is obtained instead of (240). Vlad [143] has noted that this term (with characteristic parameter $\mu_{1}$ ) can be viewed as describing the interactions between the individuals and the environment (in the sense that it sets a limit $p_{\max }$ for the population density, Eq. (238)). Clearly, such a role is not played by the terms in Eq. (217) with characteristic (age-dependent) parameters $\lambda(a)$ and $\mu_{0}(a)$. For this reason, $r_{L}$ given by Eq. (222) is sometimes called the intrinsic growth rate of the population. Assuming that $p_{0}(x, y) \ll p_{\max }$, for low enough values of $p(x, y, t)$ and $t$ Eq. (239) will be a good approximation to the full logistic evolution equation (237). This is why $r_{L}$ is sometimes also called the initial growth rate of the population number density $p(x, y, t)$.

\section{APPENDIX B. AGE-STRUCTURED DERIVATION OF SEQUENTIAL MODELS}

In Appendix A, the non-sequential evolution Eq. (1) has been derived using mortality and natality instantaneous rates $\left(\mu_{0}(a)\right.$ and $\lambda(a)$, respectively). Recently it has been shown that using rates per generation $\left(\widetilde{\mu}_{0}(a)\right.$ and $\widetilde{\lambda}(a)$, respectively) instead, a simple derivation of the sequential evolution Eq. (157) is possible [16]. To see this, we begin by noting that we can clearly consider the following sequential equation

$$
\begin{aligned}
& \rho(a+T, x, y, t+T)-\rho(a, x, y, t)= \\
& +\int_{-\infty}^{+\infty} \int_{-\infty}^{+\infty} d \triangle_{x} d \triangle_{y} \rho\left(a, x-\triangle_{x}, y-\triangle_{y}, t\right) \phi\left(\triangle_{x}, \triangle_{y}\right) \\
& -\rho(a, x, y, t) \\
& -\int_{-\infty}^{+\infty} \int_{-\infty}^{+\infty} d \triangle_{x} d \triangle_{y} \widetilde{\mu}_{0}(a) \\
& \rho\left(a, x-\triangle_{x}, y-\triangle_{y}, t\right) \phi\left(\triangle_{x}, \triangle_{y}\right) .
\end{aligned}
$$

Note that in the last term, the mortality and the dispersal effects are applied sequentially (because the integral and the dispersal kernel are included). Integrating this equation over age $(a=0$ to $a=\infty)$ and defining $a^{\prime} \equiv a+T$ we obtain

$$
\begin{aligned}
& p(x, y, t+T)-\int_{0}^{T} d a^{\prime} \rho\left(a^{\prime}, x, y, t+T\right)-p(x, y, t)= \\
& +\int_{-\infty}^{+\infty} \int_{-\infty}^{+\infty} d \triangle_{x} d \triangle_{y} p\left(x-\triangle_{x}, y-\triangle_{y}, t\right) \phi\left(\triangle_{x}, \triangle_{y}\right) \\
& -p(x, y, t) \\
& -\int_{0}^{\infty} d a \widetilde{\mu}_{0}(a) \int_{-\infty}^{+\infty} \int_{-\infty}^{+\infty} d \triangle_{x} d \triangle_{y} \\
& \rho\left(a, x-\triangle_{x}, y-\triangle_{y}, t\right) \phi\left(\triangle_{x}, \triangle_{y}\right) .
\end{aligned}
$$

The second term corresponds obviously to individuals with ages between 0 and $T$, i.e. born between $t$ and $t+T$, namely

$$
\begin{aligned}
& \int_{0}^{\infty} d a \int_{-\infty}^{+\infty} \int_{-\infty}^{+\infty} d \triangle_{x} d \triangle_{y} \widetilde{\lambda}(a) \\
& \rho\left(a, x-\triangle_{x}, y-\triangle_{y}, t\right) \phi\left(\triangle_{x}, \triangle_{y}\right),
\end{aligned}
$$

because $T$ is 1 generation and $\widetilde{\lambda}(a)$ is the birth rate per generation. Again, the natality and the dispersal effects are applied sequentially (i.e., the integral and the dispersal kernel appear). Using also Eq. (221) leads us finally to Eq. (157), where we have defined $R_{0}$ as

$$
R_{0}-1 \equiv \int_{0}^{\infty} d a\left[\widetilde{\lambda}(a)-\widetilde{\mu}_{0}(a)\right] c_{s t}(a) .
$$

It is interesting to note that the condition $R_{0}>1$, which Weinberger [112] showed that is necessary for the population not to extinguish and front solutions to Eq. (157) to exist, corresponds to the effect of natality being stronger than that of mortality, which makes biological sense.

Finally, let us mention that is possible to add a quadratic term, e.g.

$$
\begin{aligned}
& -\widetilde{\mu}_{1} \int_{-\infty}^{+\infty} \int_{-\infty}^{+\infty} d \triangle_{x} d \triangle_{y} p\left(x-\triangle_{x}, y-\triangle_{y}, t\right) \phi\left(\triangle_{x}, \triangle_{y}\right) \\
& \int_{-\infty}^{+\infty} \int_{-\infty}^{+\infty} d \triangle_{x} d \triangle_{y} \rho\left(a, x-\triangle_{x}, y-\triangle_{y}, t\right) \phi\left(\triangle_{x}, \triangle_{y}\right),
\end{aligned}
$$

to Eq. (241), which yields a logistic discrete-time reproduction function, i.e. an additional term

$-\widetilde{\mu}_{1}\left[\int_{-\infty}^{+\infty} \int_{-\infty}^{+\infty} d \triangle_{x} d \triangle_{y} p\left(x-\triangle_{x}, y-\triangle_{y}, t\right) \phi\left(\triangle_{x}, \triangle_{y}\right)\right]^{2}$

to Eq. (244). However, the speed of fronts would be the same and, more importantly, such a logistic discrete-time reproduction function is known from non-spatial models to yield negative population densities [56], which makes no physical sense (numerical simulations show that the same happens for spatial models [16]). This is the reason why, as we review in Sec. VII.B, Eq. (157) has been recently applied together with the simple assumption of a vanishing net reproduction above saturation density.

[1] J. Fort and V. Méndez, Rep. Progr. Phys. 65, 895 (2002).

[2] W. van Saarloos, Phys. Rep. 386, 29 (2003).

[3] J. Merikoski, J. Maunuksela, M. Myllys and J. Timonen, Phys. Rev. Lett. 90, 024501 (2003). 
[4] J. Fort and V. Méndez, Phys. Rev. Lett. 82, 867 (1999).

[5] J. Fort and V. Méndez, Phys. Rev. Lett. 89, 178101 (2002).

[6] A. L. Garner, Y. Y. Lau, T. L. Jackson, M. D. Uhler, D. W. Jordan and R. M. Gilgenbach, J. Appl. Phys. 98, 124701 (2005).

[7] V. S. Zykov and K. Showalter, Phys. Rev. Lett. 94, 068302 (2005).

[8] V. Ferreiro, J. F. Douglas, J. Warren, and A. Karim, Phys. Rev. E 65, 051606 (2002).

[9] B. Rosenstein, B. Ya Shapiro and I. Shapiro, Europhys. Lett. 70, 506 (2005).

[10] J. F. Douglas, K. Efimenko, S. A. Fischer, F. R. Phelan and J. Genzer, Proc. Nat. Acad. U.S. 104, 10324 (2007).

[11] J. Fort, J. Appl. Phys. 101, 094701 (2007).

[12] J. Fort and T. Pujol, New J. Phys. 9, 234 (2007).

[13] V. Méndez, S. Fedotov, D. Campos and W. Horsthemke, Phys. Rev. E 75, 011118 (2007).

[14] H. Wei, G. Lilienkamp, J. Davidsen, M. Bär and R. Imbihl, Phys. Rev. E 73, 016210 (2006).

[15] K. Davison, P. Dolukhanov, G. R. Sarson and A. Shukurov, J. Arch. Sci. 33, 641 (2006).

[16] J. Fort, J. Pérez-Losada and N. Isern, Phys. Rev. E 76, 031913 (2007).

[17] V. O. Vlad and J. Ross, Phys. Rev. E 66, 061908 (2002).

[18] V. Méndez, D. Campos and S. Fedotov, Phys. Rev. E 70, 066129 (2004).

[19] S. Fedotov and Y. Okuda, Phys. Rev. E 66, 021113 (2002).

[20] J. Fort, D. Jana and J. Humet, Phys. Rev. E 70, 031913 (2004).

[21] J. Fort, J. Pérez, E. Ubeda and J. García, Phys. Rev. E 73, 021907 (2006).

[22] V. Méndez, V. Ortega-Cejas and D. Campos, Physica A 367, 283 (2006).

[23] S. Harris, Phys. Rev. E 68, 031912 (2003).

[24] V. Ortega-Cejas, J. Fort, V. Méndez, Physica A 366, 299 (2006).

[25] J. Fort, J. Pérez-Losada, J. J. Suñol, L. Escoda, J. M. Massaneda, New J. Phys. 10, 43045 (2008).

[26] A. Yadav and W. Horsthemke, Phys. Rev. E 74, 066118 (2006).

[27] D. Brockmann and L. Hufnagel, Phys. Rev. Lett. 98, 178301 (2007).

[28] V. Mendez, T. Pujol and J. Fort, Phys. Rev. E 65 041109 1-6 (2002).

[29] R. D. Benguria, M. C. Depassier and V. Méndez, Phys. Rev. E 69, 031106 (2004).

[30] N. Vladimirova and R. Rosner, Phys. Rev. E 71, 067303 (2005).

[31] N. Vladimirova, V. G. Weirs and L. Ryzhik, Combustion Theor. \& Modelling 10, 727 (2006).

[32] J. Fort, D. Campos, J. R. González and J. Velayos, J. Phys. A: Math. Gen. 37, 7185 (2004).

[33] T. Pujol, J. Fort, J. R. González, L. Montoro, M. Pelegrí, Physica A 387, 1987 (2008).

[34] T. Pujol, J. Fort, J. R. González, L. Montoro and J. J. Suñol, submitted (2008).

[35] J. Fort, R. Nathan, G. G. Katul and N. Horvitz, submitted (2008).
[36] M. J. Hamilton and B. Buchanan, Proc. Nat. Acad. Sci. U.S. 104, 15625 (2007).

[37] J. Fort, T. Pujol and L. L. Cavalli-Sforza, Cambridge Archaeol. J. 14, 53-61 (2004).

[38] G. J. Ackland, M. Signitzer, K. Stratford and M. H. Cohen, Proc. Nat. Acad. U.S. 104, 8714 (2007).

[39] V. Ortega-Cejas, J. Fort, V. Méndez and D. Campos, Phys. Rev. E 69, 031909 (2004).

[40] S. T. Abedon and R. R. Culler, J. Theor. Biol. 248, 111 (2007).

[41] M. O. Vlad, F. Moran, M. Tsuchiya, L. L. CavalliSforza, P. J. Oefner and J. Ross, Phys. Rev. E 65, 061110 (2002).

[42] C. A. Edmons, A. S. Lillie and L. L. Cavalli-Sforza, Proc. Nat. Acad. Sci. U. S. 101, 975 (2004).

[43] M. O. Vlad, L. L. Cavalli-Sforza and J. Ross, Proc. Nat. Acad. Sci. U. S. 101, 10249 (2004).

[44] S. Fedotov and A. Iomin, Phys. Rev. Lett. 98, 118101 (2007).

[45] S. Fedotov, J. Phys. A: Math. Gen. 33, 7033 (2000).

[46] M. Bär, A. Hagbert, E. Meron and U. Thiele, Phys. Rev. Lett. 83, 2664 (1999); Phys. Rev. E 62, 366 (2000).

[47] J. E. Haus and K. W. Kehr, Phys. Rep. 150, 263 (1987), Sec. 10.

[48] P. Meakin, Phys. Rev. B 28, 5221 (1983).

[49] N. A. Hill, J. Theor. Biol. 186, 503 (1997).

[50] Note that in Ref. [1] we defined $\phi\left(\triangle_{x}, \triangle_{y}\right)$ as the probability of a jump from $\left(x+\triangle_{x}, y+\triangle_{y}, t\right)$, whereas in the present review we use negative signs instead. This is clearer for non-isotropic (i.e., biased) random walks because otherwise the kernel $\Phi(\theta)$ in Eq. (30) would correspond to a jump with angle $-\theta$, which would be rather confusing.

[51] H. C. Berg, Random walks in biology (Princeton University Press, Princeton, 1983).

[52] A. J. Lotka, Elements of mathematical biology (Dover, New York, 1956), pp. 64-69.

[53] C. A. S. Hall, Ecological Modelling 43, 5 (1988).

[54] F. van den Bosch, J.A.J. Metz and O. Diekmann, J. Math. Biol. 28, 529 (1990).

[55] J. Fort and V. Méndez, Phys. Rev. E 60, 5894 (1999).

[56] J. D. Murray, Mathematical Biology, 3rd ed., 2 vols. (Springer-Verlag, Berlin, 2002).

[57] E. A. Colding and N. A. Hill, J. Theor. Biol. 233, 573 (2005).

[58] C. O. Paulin, Atlas of the Historical Geography of the United States (Greenwood Press, Westport, 1932), plates 76-79.

[59] S. A. Flanders, Atlas of American Migration (Facts of File, New York, 1998).

[60] D. Campos, J. Fort and V. Méndez, Theor. Popul. Biol. 69, 88 (2006).

[61] J. P. Ferrie, in Historical statistics of the US, millenial edition, 5 vols, ed. S B. Carter, S. Gartner, M.R. Haines, A. L. Olmstead, R. Sutch and G. Wright (Cambridge University Press, Cambridge, 2006), Chapter Ac, table 2. See also J. P. Ferrie, Historical Methods 29, 141 (1996).

[62] For the migration data in Ref. [61] only the adult subpopulation is considered, well-known $2 \mathrm{D}$ diffusion 
theory can be applied (without reproduction terms) and we can estimate $D x$ and $U_{x}$ from $\left\langle\Delta^{2}\right\rangle /(4 T)=$ $\left\langle r^{2}\right\rangle /(4 t)=D_{x}$ (see Eq. (34)) and $\langle\Delta\rangle / T=\langle r\rangle / T=$ $\sqrt{\pi D / T}$ (see also Ref. [56], Eq. (9.10)).

[63] One can try to estimate $b$ from Eq. (30) with the minus sign and the migration data in Ref. [61] into the ENC [East North Central] region (which has more migration directions than the other regions). Those data are clearly biased, with more migrations from the West than from the East. However, the results are quite different if we estimate $b$ using the horizontal directions, than if diagonal directions are used (e.g., the latter approach gives $b=0.113$, thus $\beta=0.7$ ). This shows the need of more detailed data, i.e. in many directions, so that a fit can be made to Eq. (30) in order to try to estimate $b$ accurately. We are not aware of such detailed data, so it is more reasonable to analyze the front speed as a function of $\beta$ (Fig. 1) at this stage.

[64] A. J. Ammerman and L. L. Cavalli-Sforza, Man 6, 674 (1971).

[65] J. Zilhao, Proc. Nat. Acad. Sci. U.S. 98, 14180 (2001).

[66] K. Davison, P. M. Dolukhanov, G. R. Sarson, A. Shukurov and G. I. Zaitseva, Documenta Praehistorica 34, 139 (2007).

[67] M. H. Cohen, in Springer Proceedings in Physics, Nonlinearity with disorder, eds. F Abdullaev, A. R. Bishop and S. Pnevmatikos (Springer, Berlin, 1992), pp. 161170.

[68] K. Aoki, M. Shida, N. Shigesada, Theor. Popul. Biol. 50,1 (1996).

[69] R. A. Fisher, Ann. Eugen. 7, 353 (1937).

[70] J. Yin, Biochem. Biophys. Res. Commun. 174, 1009 (1991).

[71] M. P. Hassell, O. Miramontes, P. Rohani and R. M. May, J. Anim. Ecol. 64, 662 (1995).

[72] P.B. Sunderland, , B.J. Mendelson, Z.-G. Yuan and D.L. Urban, Comb. Flame 116, 376 (1999).

[73] S. Fedotov, Phys. Rev. Lett. 86, 926 (2001).

[74] W. Feller, An Introduction to Probability Theory and is Applications (Wiley, New York, 1968), 3rd. ed.

[75] Ya. B. Zeldovich, G. I. Barenblatt, V. B. Librovich and G. M. Makhviladze, The Mathematical Theory of Combustion and Explosions (Consultants Bureau, New York, 1985)

[76] T. Echekki, A. R. Kerstein, T. D. Dreeben , Comb. Flame 125, 1083 (2001).

[77] D. C. Haworth, T. J. Poinsot, J. Fluid Mech. 244, 405 (1992).

[78] A. R. Masri, S. S. Ibrahim and B. J. Cadwallader, Experimental Thermal and Fluid Science 30, 687 (2006).

[79] P. Naamansen, D. Baraldi, B. H. Hjertager, T. Solberg and S. Cant, Journal of Loss Prevention in the Process Industries 15, 189 (2002).

[80] J. Xin, SIAM Rev. 42, 161 (2000).

[81] R. D. Benguria and M. C. Depassier, Phys. Rev. E 57, 6493 (1998).

[82] M. Abel, A. Celani, D. Vergni and A. Vulpiani, Phys. Rev. E 64, 046307 (2001).

[83] P. D. Ronney, in Microgavity Combustion: Fires in Free Fall (Academic Press, London, 2001), p.35.
[84] U. Warnatz, U. Maas and R. W. Dibble, Combustion (Springer, Berlin, 2001).

[85] R. D. Benguria, J. Cisternas and M. C. Depassier, Phys. Rev. E 52, 44116, Issue 3, February 1999, Pages 37610 (1995).

[86] Y. B. Zeldovich and D. A. Frank-Kamenetzki, Acta Physicochim. USSR 9, 341 (1938).

[87] A. Kolmogorov, I. Petrovski and N. Piskunov, Bull. Univ. Moscow, Ser. Int. A 11 (1937).

[88] D. G. Aronson and F. H. Weinberger, Adv. Math. 30, 33 (1978).

[89] D. G. Aronson and F. H. Weinberger, in Partial differential equations and related topics, ed. J. A. Goldstein (Springer, Berlin, 1975), pp. 5-49.

[90] P. D. Ronney, in 27th Symp. (Int.) on Combustion (The Combustion Institute, Pittsburg, 1998), p. 2485.

[91] S. R. de Groot and P. Mazur, Non-equilibrium thermodynamics (Dover, London, 1962).

[92] C. J. Rutland and A. Trouvé, Comb. Flame 94, 41 (1993).

[93] P. Clavin and F. A. Williams, J. Fluid Mech. 116, 251 (1982).

[94] S. Elghobashi, R. Zhong, O. Boratav, Phys. Fluids 11, 312316, 387 (1999).

[95] F. Van Den Bosch, F. R. Hengeveld and J.A.J. Metz, J. Biogeogr. 19, 135 (1992).

[96] M. Kot, M.A. Lewis and P. van den Driessche, Ecology 77, 2027 (1996).

[97] M. Neubert and H. Caswell, Ecology 81, 1613 (2000).

[98] A. J. Ammerman and L. L. Cavalli-Sforza, The Neolithic transition and the genetics of population in Europe (Princeton University Press, Princeton, 1984).

[99] R. Pinhasi, J. Fort, A. J. Ammerman, PLoS Biology 3, 2220 (2005).

[100] J. Stauder, The Majangir. Ecology and Society of a Southwest Ethiopian people (Cambridge University Press, Cambridge, 1971).

[101] Table 5 in Ref. [100], p. 58 gives the age distribution of unmarried people for preindustrial agriculturalists which (neglecting death effects, which we cannot estimate, as a first approximation) allows to determine the number of people who have left a domestic group as $N_{1}=44, N_{2}=49, N_{3}=2, N_{4}=1$ (from which our values of $p_{i}$ follow directly), with mean ages $a_{2}=27.5$, $a_{3}=37.5, a_{4}=47.5$. Concerning $a_{1}$, in the same Ref. [100], p. 67 , it is stated that children do not become marriageable (i.e., able to disperse according to the Majangir custom) until they are $a_{1} \simeq 15$ yr old. Note that these values of $p_{i}$ and $a_{i}$ yield a mean $\langle a\rangle \simeq 24 \mathrm{yr}$, which (adding about $1 \mathrm{yr}$ from the parents migration until the child's birth) is indeed consistent with the value of 25 yr for the generation time used in Refs. [98,4]. However, a final correction is necessary. If a son/daughter leaves their parents when he is, e.g., $19 \mathrm{yr}$ old, to this we should add the time interval from the migration of his parents until his/her birth. Since the mean number of children per family for preindustrial agriculturalists is about 6.5 [102] and their average birth interval is close to 2.5 yr (Ref. [98], p. 66), we find that the mean time interval from the migration of the parents until a 
child's birth is of about 8 yr. Therefore, we have used $T_{i}=a_{i}+8 \mathrm{yr}$ to obtain our values of $T_{i}$ in the main text, and $\langle T\rangle \simeq\langle a\rangle+8 \mathrm{yr} \simeq 32 \mathrm{yr}$.

[102] D. W. Sellen, R. Mace, Current Anthropology 38878 (1997), table 2.

[103] Á. Cartea, D. del-Castillo-Negrete, Phys. Rev. E 76, 041105 (2007).

[104] N. Shigesada and K. Kawasaki, Biological invasions. Theory and practice (Oxford University Press, Oxford, 1997).

[105] M. Arim, S. R. Abades, P. E. Neill, M.o Lima, P. A. Marquet, Proc. Nat. Acad. Sci. U.S. 103, 374 (2006).

[106] M. Delbrück, Biol. Rev. Proc. Cambridge Philos. Soc. 21, 30 (1946).

[107] H. Fricke, Phys. Rev. 24, 575 (1924).

[108] J. Yin, J. Bacteriol. 175, 1272 (1993).

[109] J. Yin and J. S. McCaskill, Biophys. J. 61, 1540 (1992).

[110] P. Dieterich, R. Klages, R. Preuss and A. Schwab, Proc. Nat. Acad. Sci. U.S. 105, 459 (2008).

[111] J. Klafter, A. Blumen and M. F. Shlesinger, Phys. Rev. A 35, 3081 (1987).

[112] H. F. Weinberger, in Nonlinear partial differential equations and applications. J. Chadam, Ed. (Springer, Berlin, 1978).

[113] H. F. Wienberger, SIAM J. Math. Anal. 13, 353 (1982).

[114] J.S. Clark, Am. Nat. 152, 204 (1998), specially Fig. 1 and p. 219.

[115] See Ref. [97], specially Fig. 15.

[116] R. Nathan, G.S. Katul, H.S. Horn, S.M. Thomas, R. Oren et al. Nature 418, 409 (2002).

[117] R. Nathan and G.G. Katul, PNAS 102, 8251 (2005).

[118] M.B. Soons, G.W. Heil, R. Nathan and G.G. Katul, Ecology 85, 3056 (2004).

[119] Note that Eq. (157) does not include saturation. We have checked that including saturation in the $2 \mathrm{D}$ simulations, the front speeds are the same. Obviously the same happens in the CSRW and DSRW approaches after linearization.

[120] According to field observations in sites close to those where the dispersal kernel was measured, the fecundity $f$ of this species is of the order $10^{4}$ seeds dispersed/(tree-yr), and its post-dispersal seed-to-adult survival probability is $s \simeq 0.06 \%$ (see [116] and citations 25 and 26 therein). Thus the net reproductive rate, $R_{0}=f s$, has been estimated in the range $6-60$ seeds/(tree-yr). The age at first reproduction (generation time) of the same species is $T \simeq 20 \mathrm{yr}$ ( [114], table $1)$.

[121] The numerical simulations agree with Eq. (157) but not with Eq. (1) with e.g. $R[p(x, y, t)]=R_{0} p(x, y, t)$.

[122] The minimum speed [Eqs. (163)-(165) for the CSRW; Eq. (169) for the DSRW] is that of the front: this is seen by comparing to simulations [full curve in Fig. 15 for the CSRW; rhombs in Fig. 16 for the DSRW].

[123] The CSRW uses $\varphi(\Delta)$, which is the kernel found in [116]. But the simulations and DSRW use $\phi(\Delta)$. Otherwise the agreement in Figs. 15 and 16 cannot be attained. For the DSRW, see specially Eq. (167).

[124] Several days of computing time are not enough for the bimodal kernel; in contrast, for the kernel $\varphi_{S}(\Delta)$ the necessary computing time is about 30 minutes (these results have been obtained for a virtual grid of $1000^{2}$ nodes and using a personal computer with an Intel Pentium III, $393 \mathrm{~KB}$ RAM and $1133 \mathrm{MHz}$ ).

[125] In Eq. (174), we have assumed for simplicity that reproduction takes place at $\left(x^{\prime}, y^{\prime}, t^{\prime}\right)$, so that $p\left(x^{\prime}, y^{\prime}, t^{\prime}\right)=$ $p\left(x-\triangle_{x}, y-\triangle_{y}, t\right)$ according to Fig. 17(c). Equation (174) can be easily generalized to the case of several possible locations of the reproductive event (using a probability distribution that reproduction takes place at $\left.\left(x^{\prime}, y^{\prime}, t^{\prime}\right)\right)$, and also to the case of several possible reproductive events during a single migration. The result is the same in all cases, namely Eq. (176).

[126] Note that the term -1 within the parentheses cannot be omitted in the definition (175) because, otherwise, in order to obtain Eq. (176) one would have to omit the first term in the RHS of, e.g., Eq. (174), but then the locations of the origin of migration $\left[\left(x-\triangle_{x}, y-\triangle_{y}, t\right)\right.$, first term in the RHS of Eq. 174)] and reproduction $\left[\left(x^{\prime}, y^{\prime}, t^{\prime}\right)\right.$, last term] would no longer be different, as required for case $(c)$. In this sense, we can say that the physical origin of the term -1 in Eq. (175) is the migration of the parents (first term in the RHS of Eqs. (172)-(174)).

[127] A comparison does not seem possible to us (in the unbiased case) because have been unable to find a mathematical derivation showing that the speed (180), with $R_{0}$ given by (184), is always higher than (17) for arbitrary values of $T, r_{L}, D$ and $U_{x}$.

[128] J. P. Birdsell, Cold Spring Harbor Symp. Quant. Biol. 22, 47 (1957).

[129] The value of $r$ is computed so that the mean-squared displacement yields the observed value (namely 1544 $\mathrm{km}^{2}$ [4]), i.e. $\left(1-p_{e}\right) r^{2}=\left\langle\Delta^{2}\right\rangle=1544 \mathrm{~km}^{2}$.

[130] One could estimate the speed in other directions. However, such substantially more tedious work is unnecessary because of the satisfactory agreement between the CSRW (full line in Fig. 19) and the mean of the simulations (triangles).

[131] Note that the generation time in Ref. [4] was estimated as $T=25 \mathrm{yr}$, directly from Ref. [98]. But In Sec. VI.B in this review it has been noted that the value $T=25$ $\mathrm{yr}$ is essentially the mean age difference between parents and their oldest son or daughter (not the mean among all of them), so that a more precise estimation is $T=32$ yr. This is why the HRD curve in Fig. 19 in the present paper for the value $r_{L}=0.03 \mathrm{yr}^{-1}$, considered in [4] (i.e., for $R_{0}=\exp \left[r_{L} T\right]=2.61$, see Eq. (184)), yields a slower front than that in Ref. [4] $(0.81 \mathrm{~km} / \mathrm{yr}$ versus $0.99 \mathrm{~km} / \mathrm{yr}$ ).

[132] In this review we assume a fixed value for the diffusion coefficient $D=\left\langle\Delta^{2}\right\rangle /(4 T)$, namely that obtained from the values of $\left\langle\Delta^{2}\right\rangle$ and $T$ implied by the observed dispersion data (for a given persistence $p_{e}$, the value of $r$ is obtained from $\left(1-p_{e}\right) r^{2}=\left\langle\Delta^{2}\right\rangle$ [129]). An alternative would be to analyze the effect of $p_{e}$ on $D$ or $\left\langle\Delta^{2}\right\rangle=\left(1-p_{e}\right) r^{2}$, but then we would have to assume a fixed value for $r$ instead (for example, the mean displacement $\langle\Delta\rangle$ implied by the observed dispersion data). But we think that the value of $D$ or $\left\langle\Delta^{2}\right\rangle$ is more likely to 
be similar for different human populations, rather than the value of $\langle\Delta\rangle$ (because the former is more useful than the latter to describe dispersal data at least in some situations, i.e. those in which the diffusion approximation is valid).

[133] A. J. Ammerman and L. L. Cavalli-Sforza, Man 6, 674 (1971).

[134] V. Méndez, J. Fort and J. Farjas, Phys. Rev. E 60, 5231 (1999), Sec. V.

[135] $R_{0 P}=\exp \left[r_{L P} T\right]$ from Eq. (184) and we use the mean value $r_{L P}=0.022 \mathrm{yr}^{-1}$ from Ref. [37].

[136] M. Currat and L. Excofier, Proc. Roy. Soc. B 272, 679 (2005).

[137] The fastest possible front speed along the horizontal or vertical directions of the square lattice will obviously be $r / T=50 \mathrm{~km} / 32 \mathrm{yr}=1.56 \mathrm{~km} / \mathrm{yr}$ (recall that $r$ is the distance between two nearest nodes, and $T$ the time between two successive jumps). This limit should be obtained for sufficiently high values of $R_{0 N}$, so that the front propagation becomes diffusion-limited. Similarly, the fastest possible front speed along the diagonal directions of the square lattice will obviously be $r \sqrt{2} /(2 T) \simeq 50 \mathrm{~km} /(\sqrt{2} 32 \mathrm{yr})=1.10 \mathrm{~km} / \mathrm{yr}$, which agrees with the asymptotic behavior of the diagonal results (squares and + crosses) observed in Fig. 22 for $R_{0 N}=3.0$.

[138] From Eq. (184), $r_{L N}=\left(\ln R_{0 N}\right) / T$ yields the estimations $r_{L N}=0.034 \mathrm{yr}^{-1}$ for $R_{0 N}=3.0$ and $r_{L N}=0.015$ $\mathrm{yr}^{-1}$ for $R_{0 N}=1.6$. This includes the range estimated from fits to Eq. (238) or (240) in Refs. [4,99], namely $0.029 \leq r_{L N} \leq 0.035 \mathrm{yr}^{-1}$ or $2.53 \leq R_{0 N} \leq 3.06$.

[139] A. J. Ammerman, in The widening harvest: The Neolithic transition in Europe-Looking back, looking forward, ed. A. J. Ammerman and P. Biagi (Archaeological Institute of America, Boston, 2003), pp. 3-23.

[140] N. M. Ferguson et al, Nature 425, 681 (2003).

[141] H. G. Othmer, S.R. Dunbar ad W. Alt, J. Math. Biol. 26, 263 (1998), specially pp. 268-270.

[142] M. O. Vlad and V. T. Popa, Math. Biosci. 76, 161 (1985).

[143] M. O. Vlad, J. Theor. Biol. 126, 239 (1987).

[144] A. J. Lotka, Théorie analytique des associations biologiques, vol. II (Hermann, Paris, 1939).

[145] J. D. Callen, Fundamentals of plasma physics (draft freely available in the internet), Appendix B, Eqs. (F.5.b,k) and (F.11.g,n). Note that the variable $i \omega$ in this reference is equivalent to $-s$ in Ref. [146] and the present review.

[146] M. R. Spiegel, Mathematical handbook of formulas and tables (McGraw-Hill, NewYork, 1970), formulae 32.25 and 32.13 .

\section{FIGURE CAPTIONS}

Fig. 1 Predicted speeds for the human invasion front of the United States in the XIX century, as a function of the random walk bias $\beta$ in the migration of individuals. The observed speed range is shown as a hatched rectangle.

Fig. 2 Invading front of Neolithic farmers $(F)$ into a region originally full of Paleolithic hunter-gatherers $(H)$, and formation of a cultural boundary. Population densities (relative to their maximum possible values) of invading Neolithic farmers $(F)$, indigenous Paleolithic hunter-gatherers $(H)$ and Paleolithic populations converted into farmers $(X)$ for $t_{1}=250 \mathrm{yr}, t_{2}=500$ yr, $\ldots, t_{10}=2500 \mathrm{yr}$, from Eqs. (43) with initial conditions $\left(n_{F}, n_{H}, n_{X}\right)=(1,0,0)$ at $r=0$ and $\left(n_{F}, n_{H}, n_{X}\right)=(0,1,0)$ elsewhere. The parameter values used are $p_{\max F} / p_{\max H}=p_{\max X} / p_{\max H}=50$, $\tau_{F}=\tau_{X}=\tau_{H}=18.3 \mathrm{yr}, D=7 \mathrm{~km}^{2} / \mathrm{yr}, \gamma=0.0005$ $\mathrm{yr}^{-1}$ and $\lambda=0.2 \mathrm{yr}^{-1}$, as suggested in Ref. [38].

Fig. 3 Front profiles for $r=0.1$ and the Laplacian kernel with $\alpha^{2}=700$. In model B $\lambda=1 / \tau$, with $\tau=2$. Note that model B yields a faster front, which also has a wider reaction zone.

Fig. 4 Front width versus delay time. The rhombs are the results from the numerical simulations, and the curves are the theoretical predictions. In model $\mathrm{B}, \lambda=$ $1 / \tau$ so that model $\mathrm{B}$ is a first-order approximation to the full dynamics described by model $\mathrm{A}$. The front speed is proportional to the front width $L$ (see Eq. (76)).

Fig. 5 Comparison between the predicted bounds (curves) and the speeds of combustion fronts obtained from numerical integrations of Eqs. (79)-(80) (circles), for several values of the dimensionless room temperature $\theta_{0}$. Lower bounds plotted are those from the BCD and ZFK methods. The KPP case gives values well below the lower value shown in the $y$-axis. Upper bounds are those from the BD and AW methods. The combustion dimensionless parameter is $C \equiv c_{p} E_{a} /(R Q)=0.5$.

Fig. 6 Upper bound (solid line) and lower bound (dotted line) for the dimensionless propagation speed of the flame front obtained from Eq. (117) and Eq. (118), respectively, as a function of the Lewis number at room temperature, without background flow $(U=0)$ and with temperature-independent transport coefficients $(\alpha=0, \beta=0)$. Circles are the front speeds from numerical integrations of Eqs. (105)-(106). In Eq. (118), the function $g(n)=\sqrt{1-n}$ has been used. The parameter values are $C=0.5$ and $\Delta c_{p} / c_{p}=0.5$.

Fig. 7 Comparison between lower and upper bounds for the propagation speed of the flame and the values obtained by numerical simulations of the full model (105)-(106), as a function of $\alpha$ for different values of the Reynolds number Re in a combustion model with no mass diffusion $\left(\mathrm{Le}_{0}=0\right)$.

Fig. 8 Contours of the propagation speed of the flame obtained by numerical simulations of the full model (solid lines) and from the upper bound Eq. (117) (dashed lines) as a function of $\alpha$ and $\beta$. Re $=0$ (background flow at 
rest) and $\mathrm{Le}_{0}=1$. The hatched region corresponds to $\beta>\beta_{c}$, so the method used to derive the upper bound breaks down.

Fig. 9 As in Fig. 8 but for $\mathrm{Re}=1000$.

Fig. 10 This figure is useful in understanding why the effective delay time $\widetilde{T}$ of the random walk depends not only on the mean $\langle T\rangle$ but also on the dispersion $\varepsilon$ of the delay time distribution. Both distributions depicted have the same mean delay $\left\langle\Delta^{2}\right\rangle$. But that in $(a)$ has a greater dispersion $\varepsilon$, implying that a few particles (or individuals) jump (or migrate) sooner (low values of $T$ ), which must lead to a faster front, i.e. to a lower effective delay $\widetilde{T}$. This explains that $\widetilde{T}$ decreases with increasing values of the dispersion $\varepsilon$, as predicted by Eq. (132).

Fig. 11 Predictions for the speed of the wave of advance of farmers in the Neolithic transition according to the model with a single delay (dashed curves) and to the more realistic model with several discrete delays (full curves). The predictions of both the multidelayed model and the single-delayed one are consistent with the front speed from the archaeological data $(1.0 \pm 0.2 \mathrm{~km} / \mathrm{yr})$ in this particular case. However, the multidelayed model is more accurate, and its corrections relative to the singledelayed model are large (about 20\%).

Fig. 12 Virus concentration versus time in an homogeneous medium of cells infected at $t=0$. The fit to the main plot is a logistic. Its time derivative (inset, full curve) makes it possible to note that a Gaussian (dotted curve) is a good description to the waiting-time distribution of the $\mathrm{T} 7$ bacteriophage.

Fig. 13 Predictions from the continuous-distribution waiting-time model (curves) versus experimental data (symbols with error bars) for the front speeds of T7 viruses infecting $E$. Coli bacteria.

Fig. 14 A filled circle represents a tree, and each empty circle stands for a seed produced by it. Reproduction is represented by the dashed arrow, dispersal by the continuous arrow. (a) Non-sequential model, Eq. (1). (b) Sequential model, Eq. (157). Model (b) is more realistic than $(a)$, because in model $(a)$ the parent tree disperses away, instead of its seeds. Therefore, in Sec. VII the sequential Eq. (157) is applied to tree species instead of Eq. (1).

Fig. 15 Front speed in 2D versus net reproductive rate, for an unimodal short-distance kernel $\varphi_{S}(\Delta)$ [11]. Stars: 2D computer simulations. Full curve: analytical 2D CSRWs, Eqs. (163)-(165). There is good agreement. The 1D speed for the same kernel is included for comparison (dotted curve). For bimodal kernels it is found that computer simulations cannot yield accurate results within a reasonable computing time, but the DSRW model overcomes this limitation (Fig. 16).

Fig. 16 Front speeds in 2D versus net reproductive rate [11]. Curves: CSRWs, Eqs. (163)-(165). Symbols: DSRWs, Eq. (169), using the values of $D_{L}$ and/or $D_{S}$ in the legend (in meters) and the corresponding kernel(s). The bimodal kernel for the yellow poplar, from Ref. [116], leads to the middle curve. It thus predicts speeds of about $10^{2}-10^{3}$ meter/generation (in contrast, the shortrange unimodal kernel (lower curve and stars, the same as in Fig. 15) predicts front speeds several orders of magnitude lower). This may solve Reid's paradox.

Fig. 17 A filled circle represents a couple of parents (a father and a mother) and each empty circle stands for one of their sons or daughters. (a) Migration before reproduction, Eq. (172). (b) Reproduction before migration, Eq. (173). (c) Reproduction during migration, Eq. (174).

Fig. 18 Predicted speeds for the human invasion of the United States in the XIX century, as a function of the random walk bias $\beta$ in the migration of individuals. The speeds shown are that according to the non-cohabitation biased model, Eq. (17), and to the cohabitation biased model, Eq. (180). The observed speed range is shown as a hatched rectangle.

Fig. 19 Neolithic front speed in 2D versus net population reproductive rate. The numerical simulations lead to different speeds in the horizontal or vertical directions (circles) than in the diagonal directions (squares), but their average (triangles) agrees with the CSRW. The DSRW results ( $x$ and + crosses) agree perfectly with the corresponding simulations

Fig. 20 Jump of individuals (or particles) for the square lattice in the simulations and the kernel (186), from an initial point $\mathrm{P}$. Continuous arrows correspond to the first generation, whereas dotted arrows correspond to the second generation.

Fig. 21 The effect of dispersion persistence on the speed of the Neolithic transition. As in Fig. 19, the CRD equation is seen to be a better approximation than the HRD equation (which was used in Ref. [4]).

Fig. 22 Predicted speeds as a function of the interaction parameter $\gamma$ between the invading species $N$ (farmers) and the indigenous species $P$ (hunter-gatherers). They are seen to be consistent with the observed speed of the Neolithic transition in Europe, namely $0.6 \leq c \leq 1.3$ $\mathrm{km} / \mathrm{yr}[99]$. 


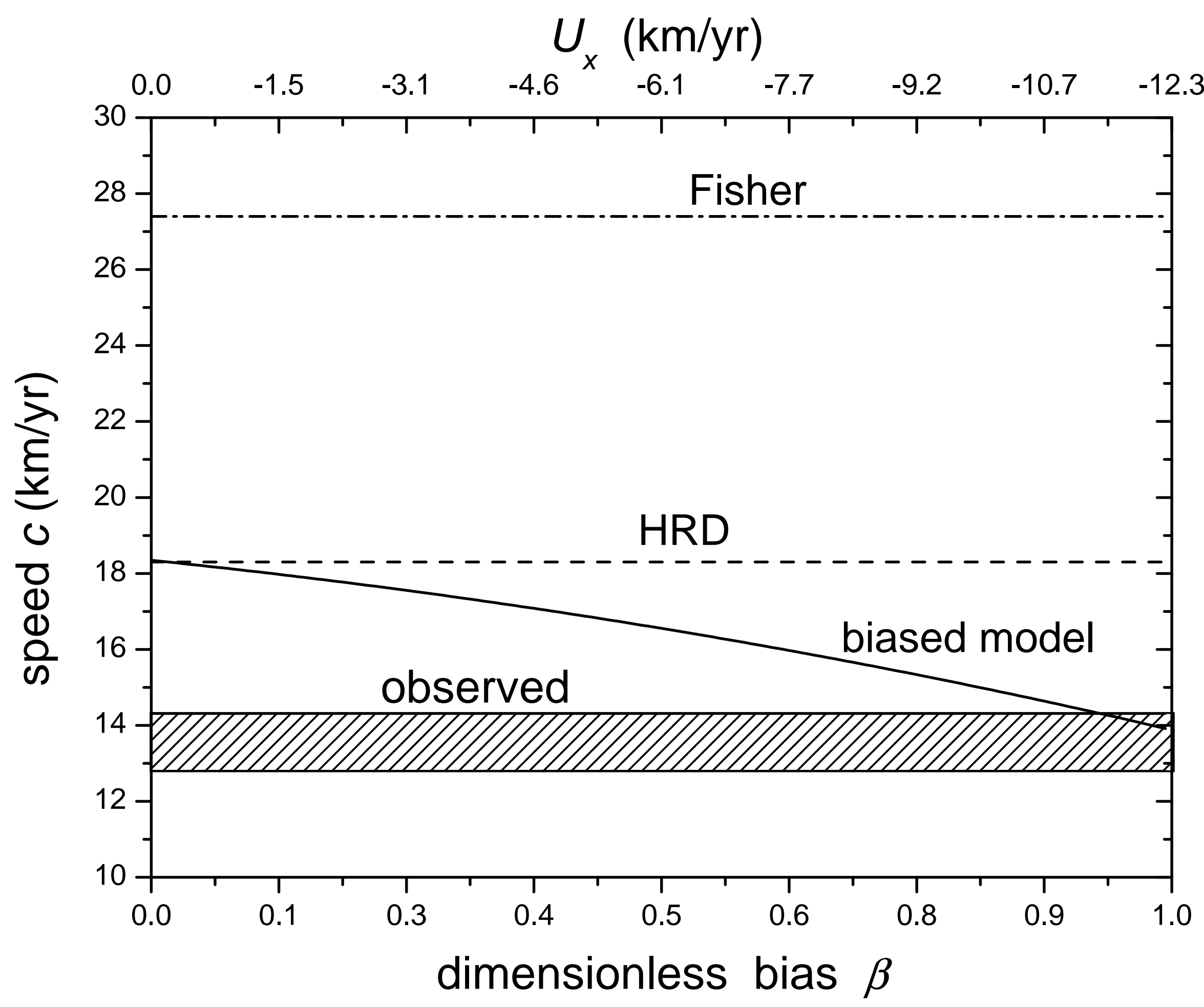




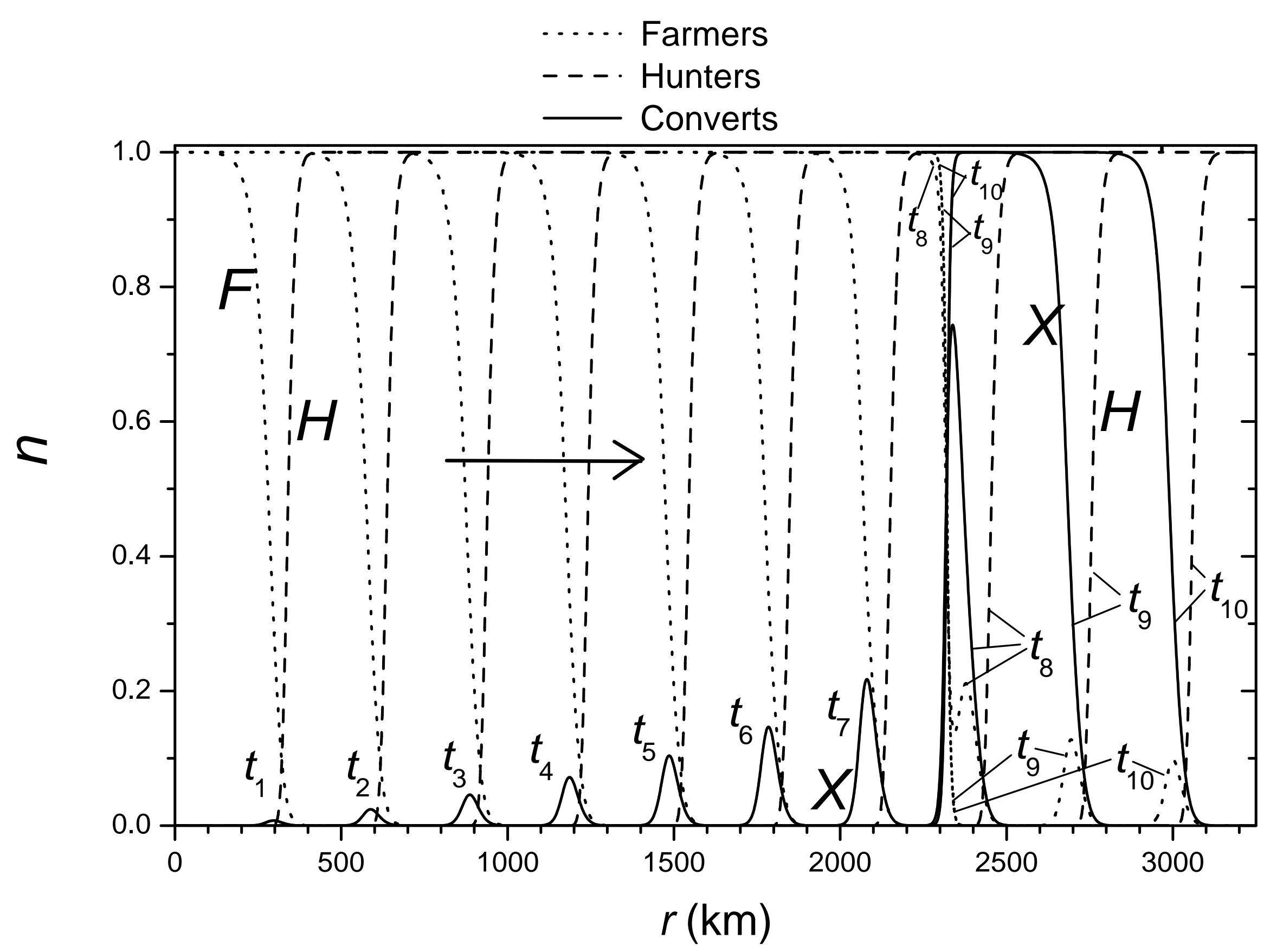


Fort and Pujol, Fig. 3

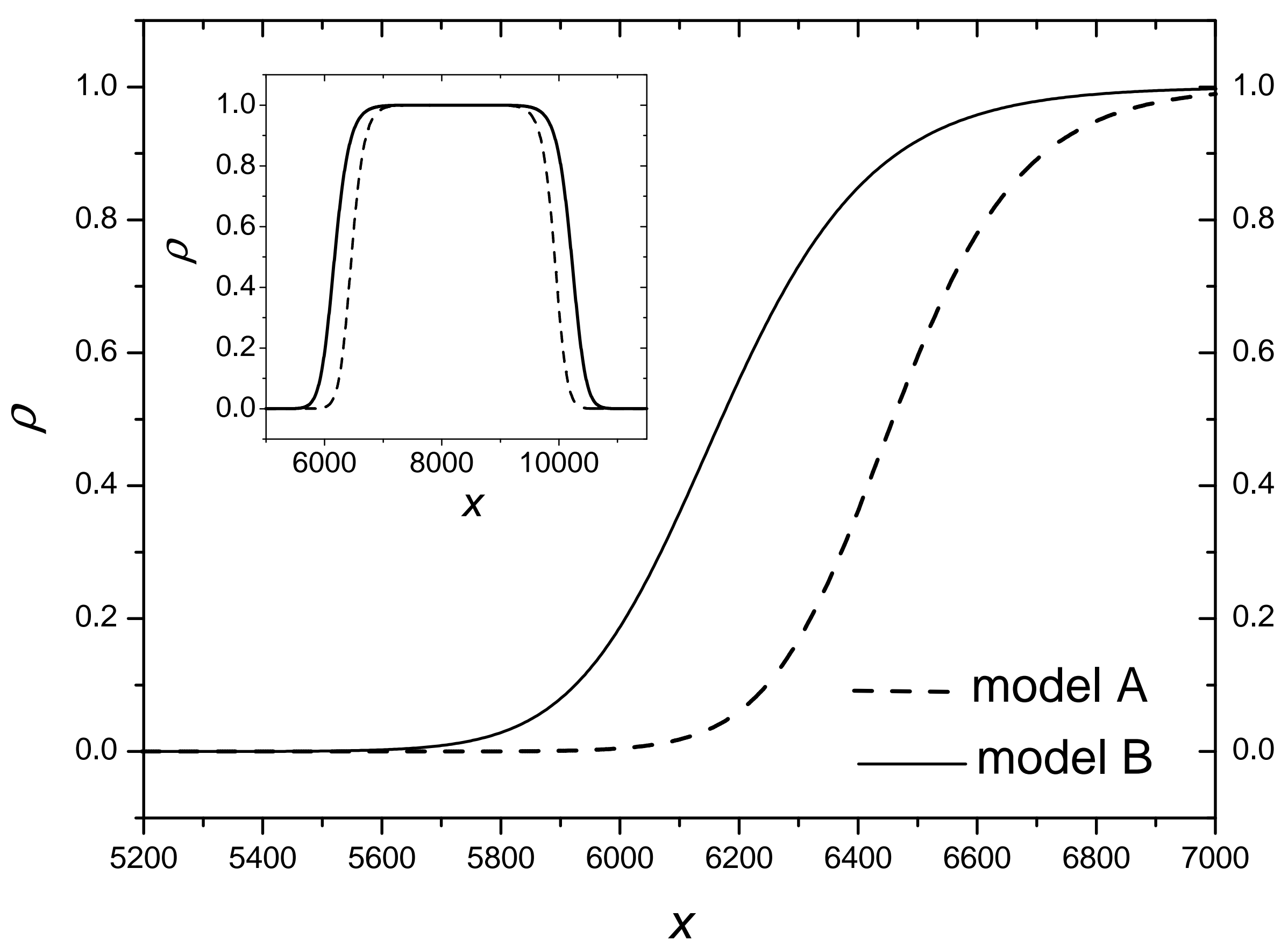


Fort and Pujol, Fig. 4

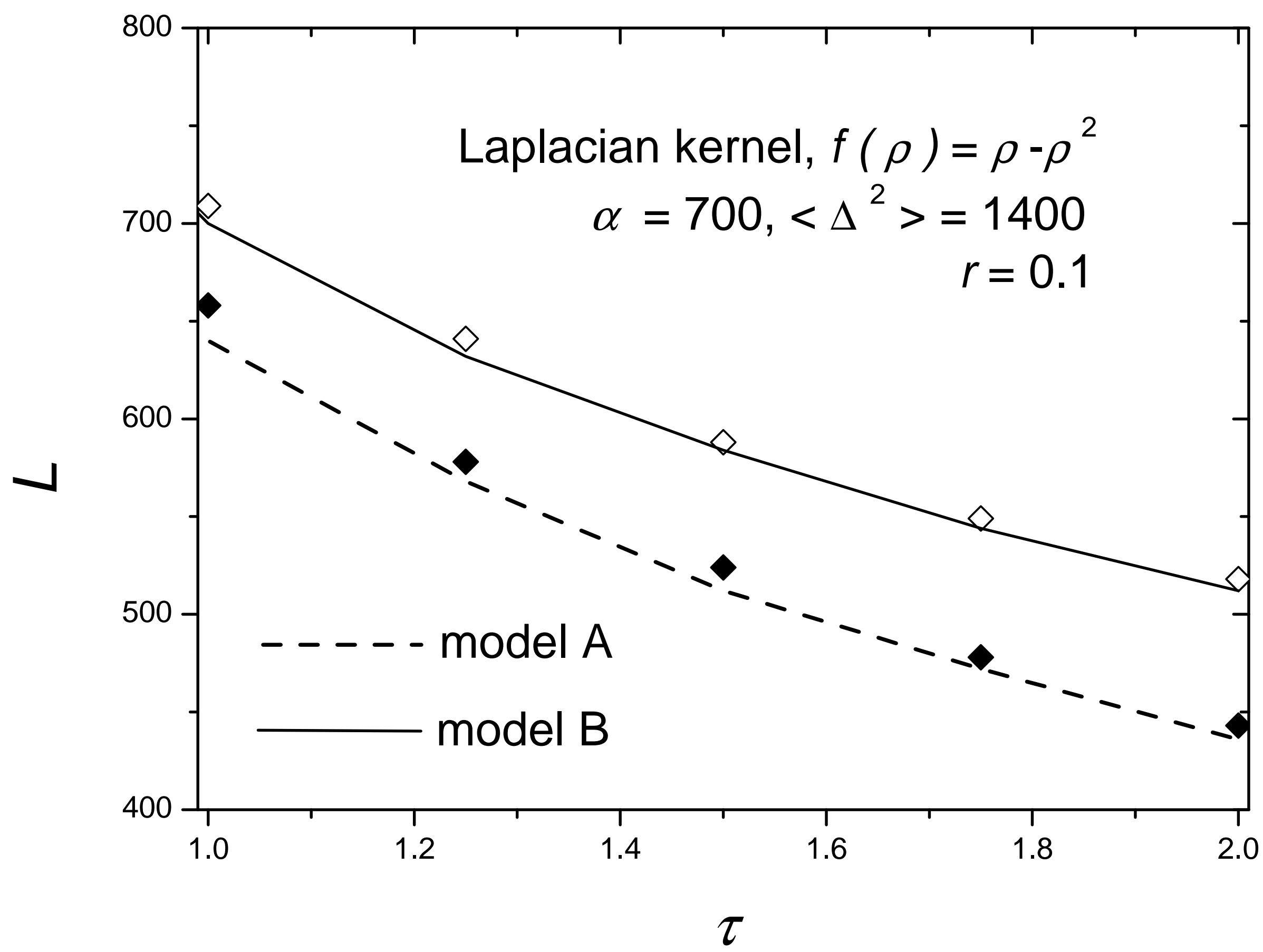


Fort and Pujol, Fig. 5

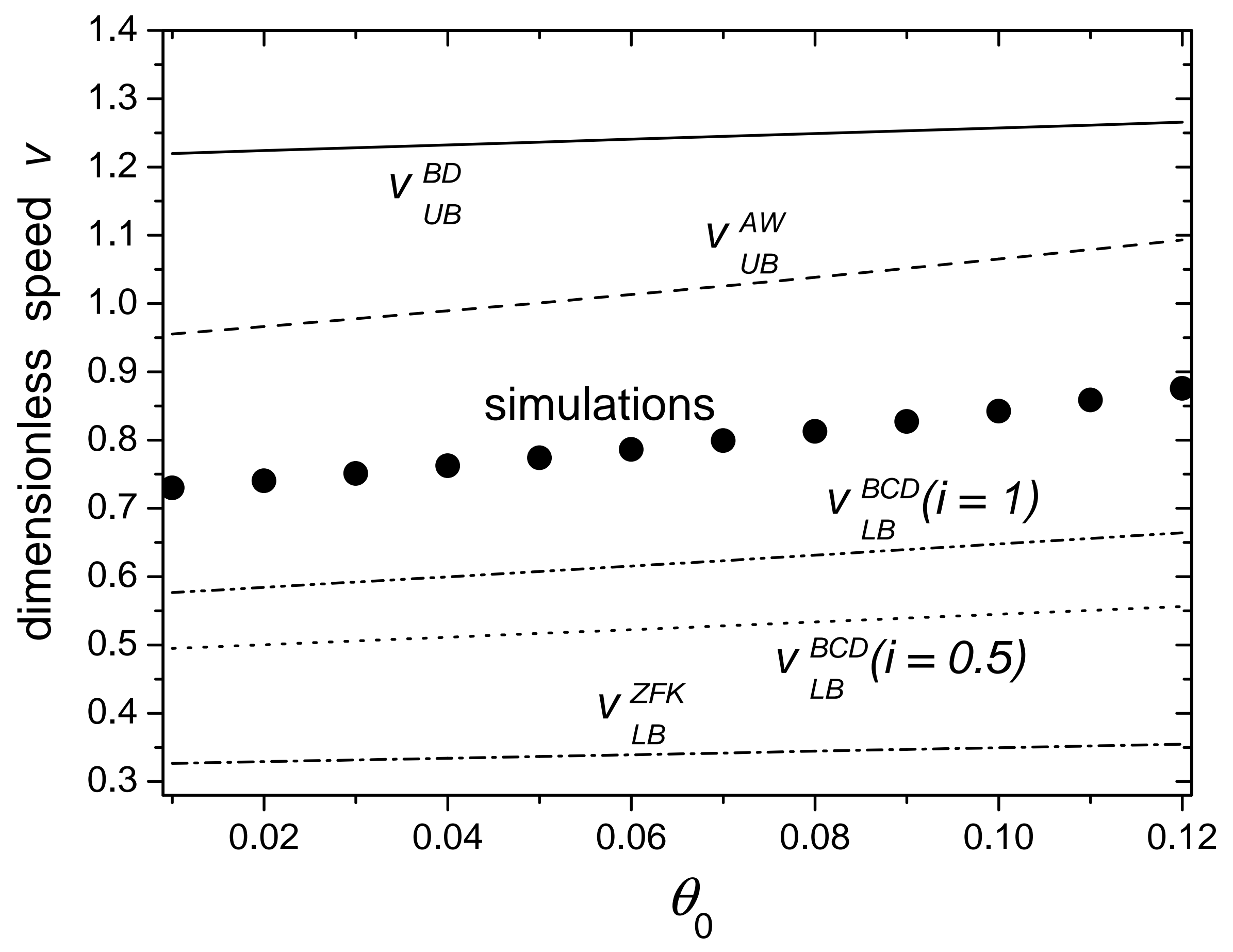


Fort and Pujol, Fig. 6

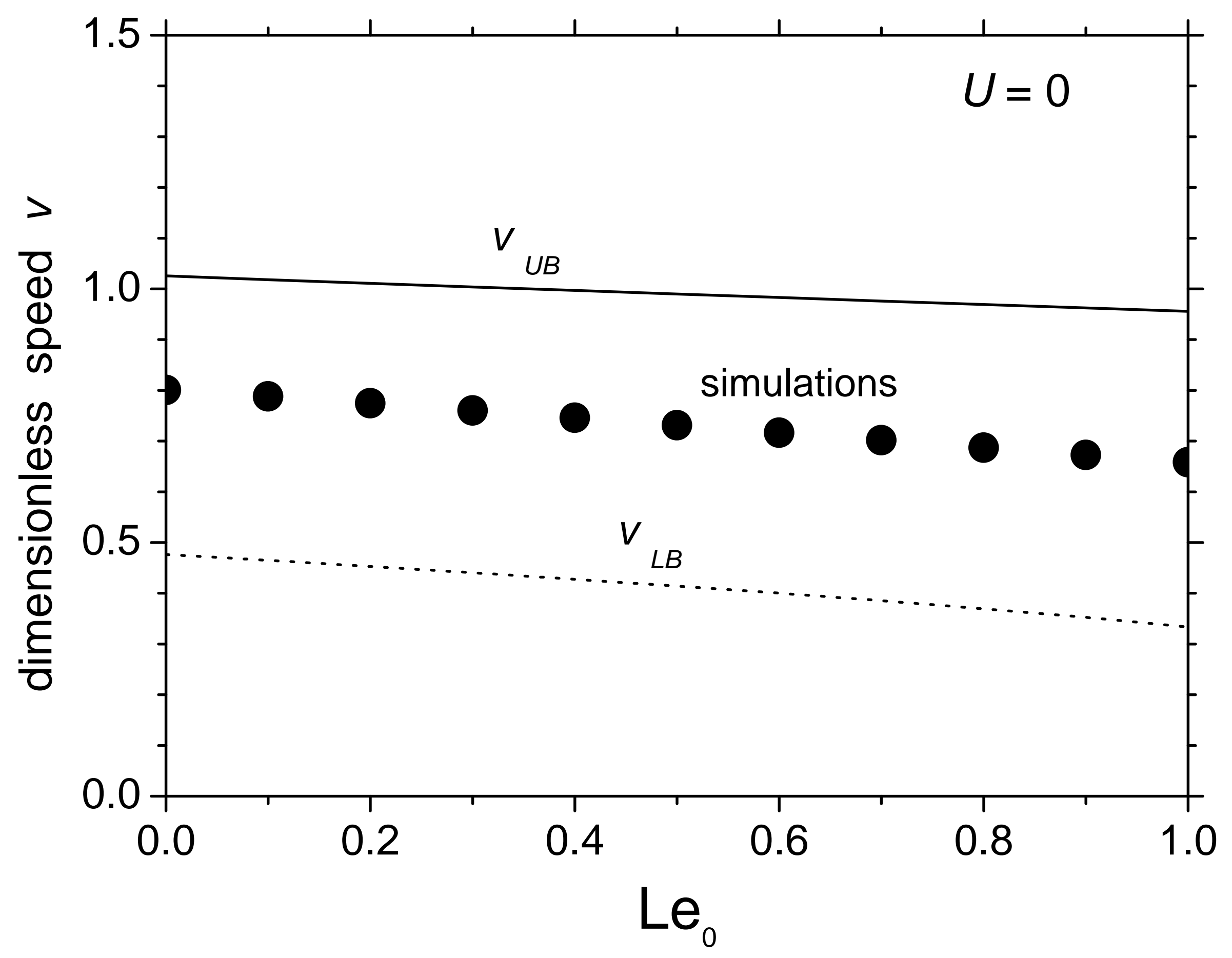


Fort and Pujol, Fig. 7

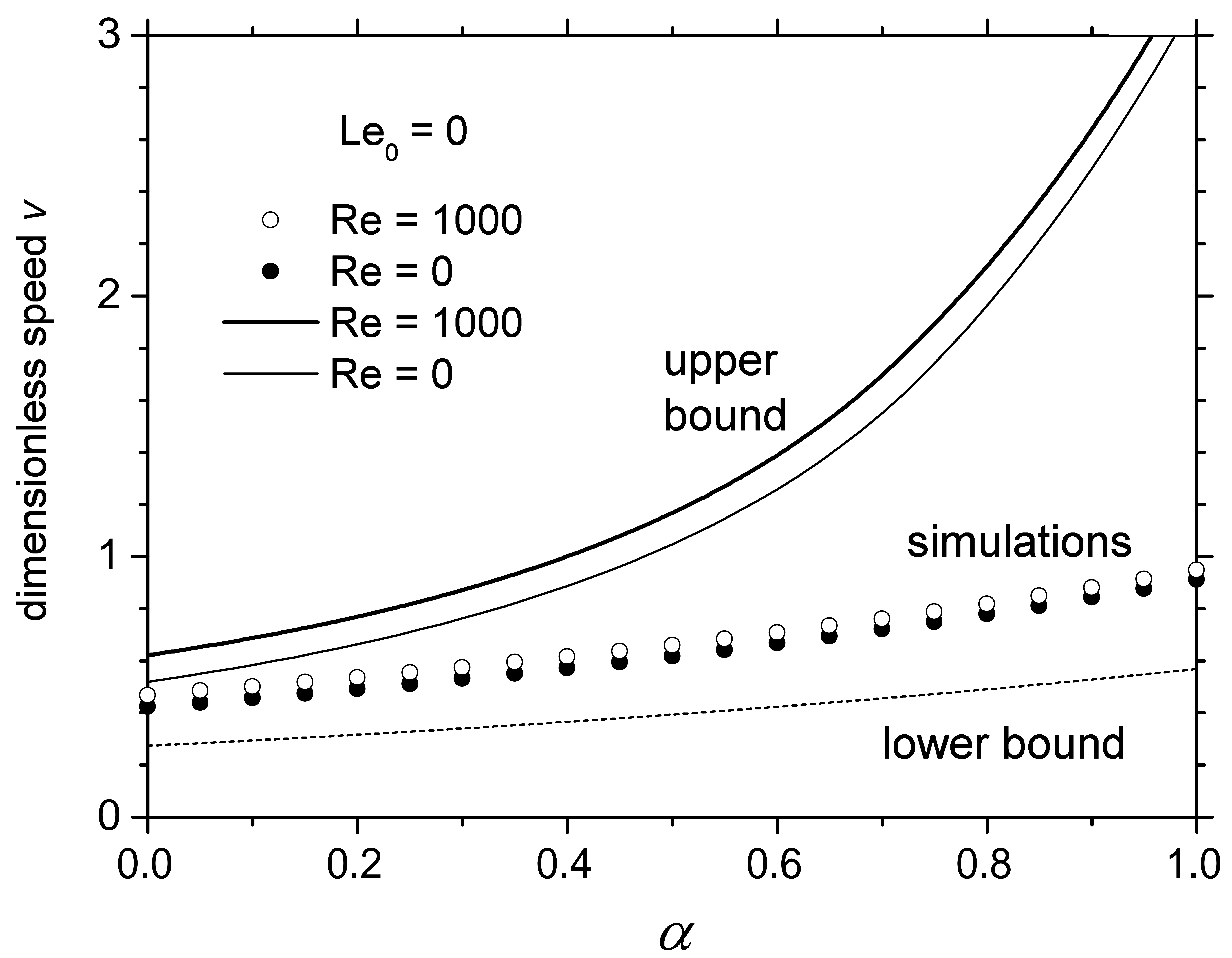


Fort and Pujol, Fig. 8

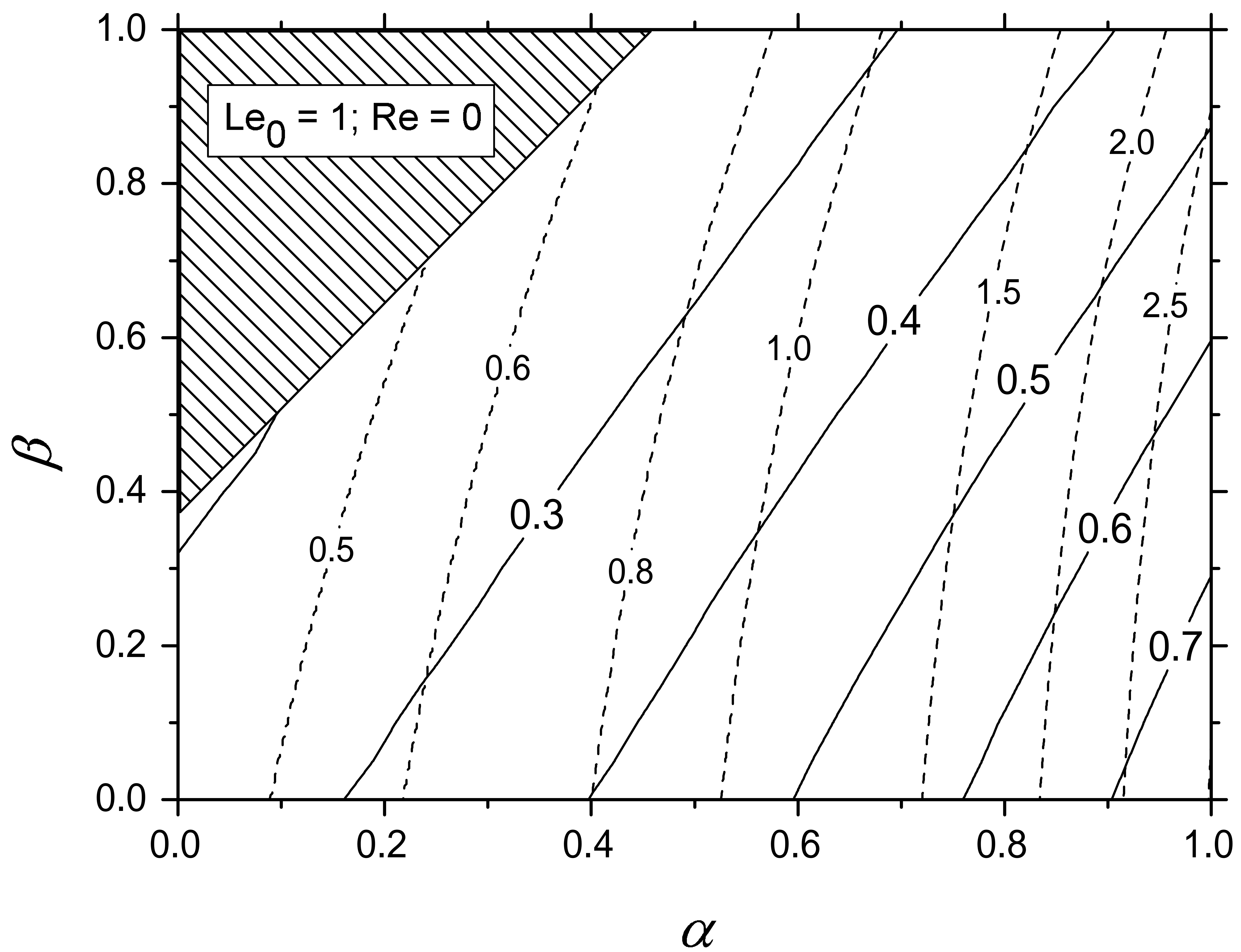


Fort and Pujol, Fig. 9

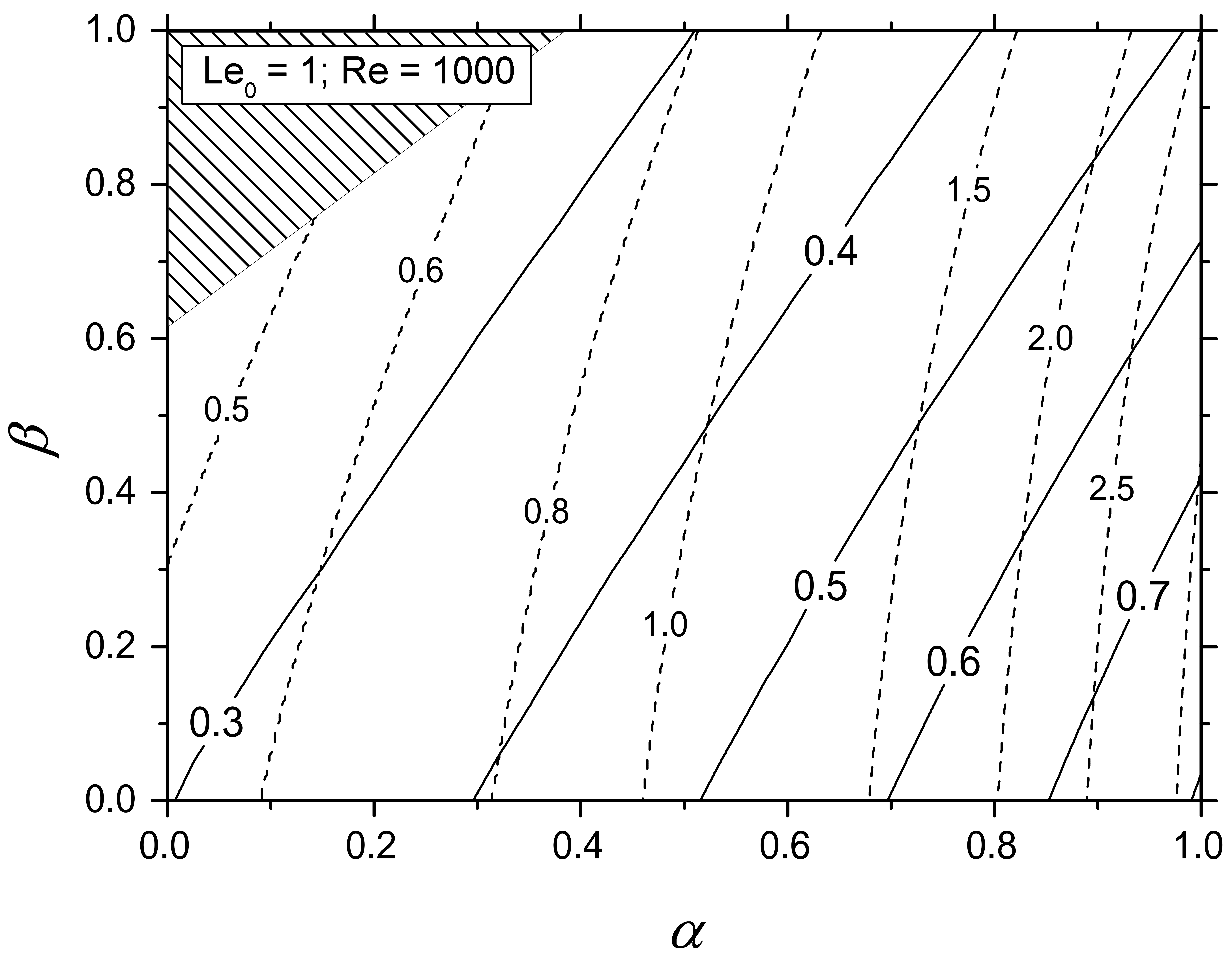


Fort and Pujol, Fig. 10.a

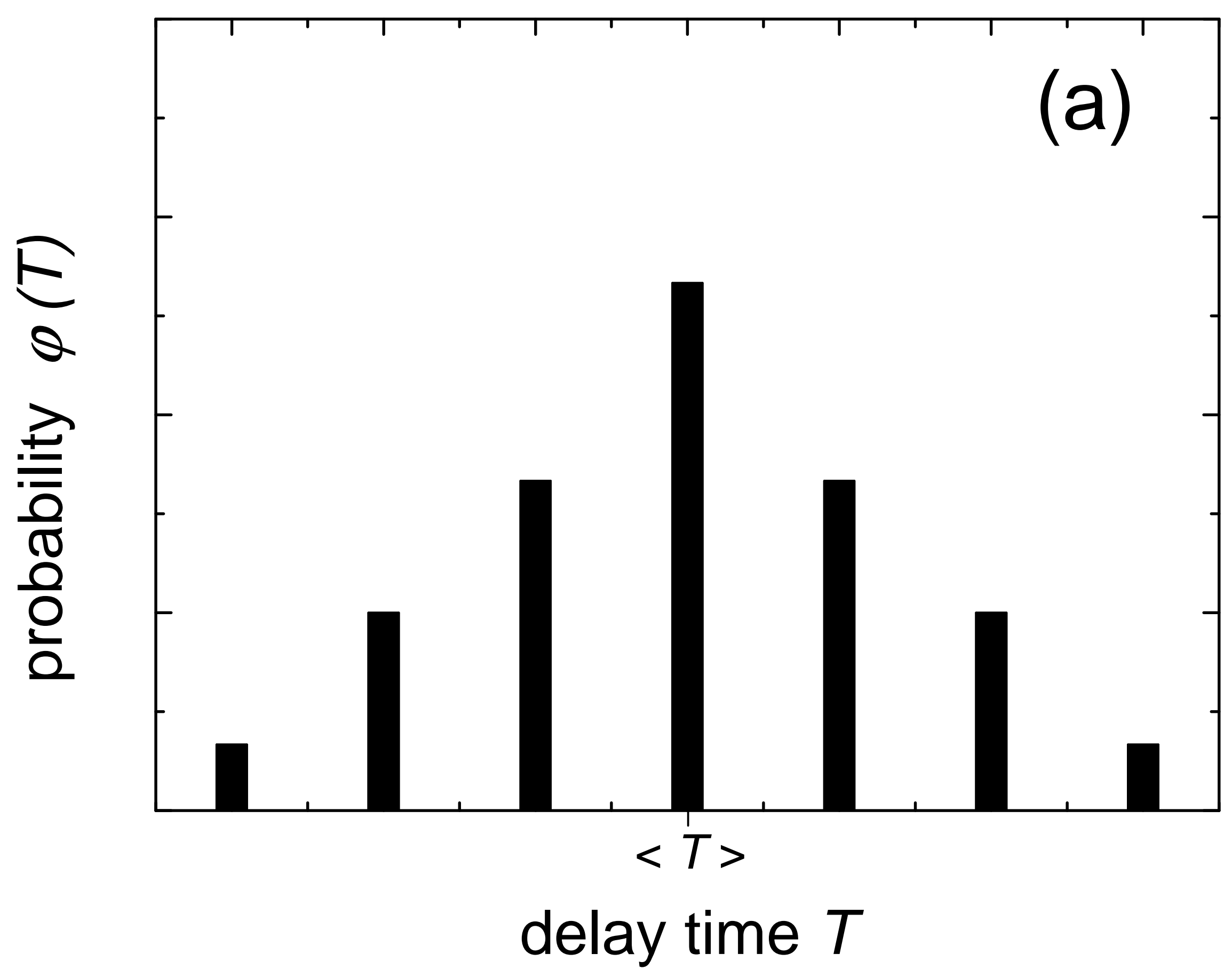


Fort and Pujol, Fig. 10.b

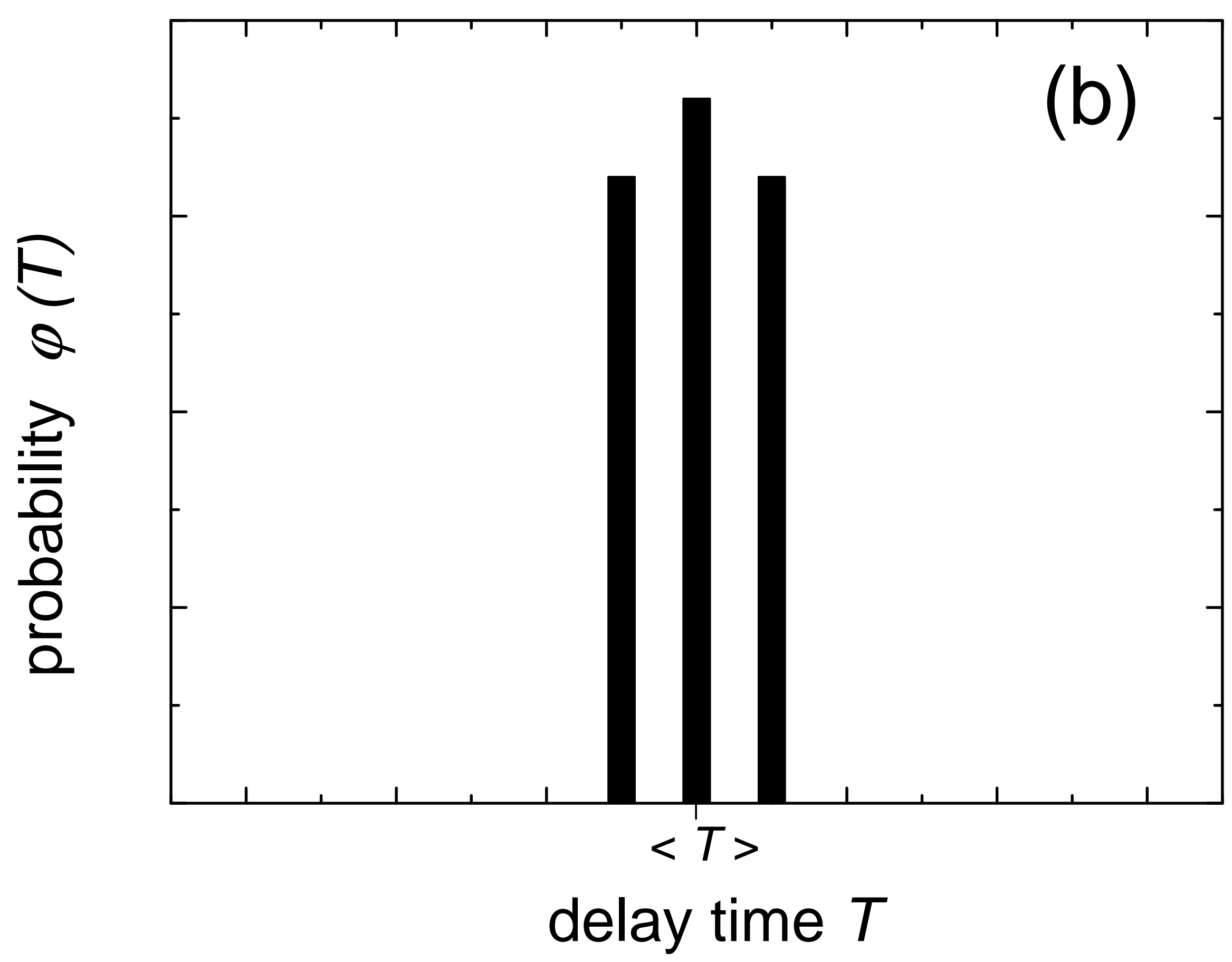


Fort and Pujol, Fig. 11

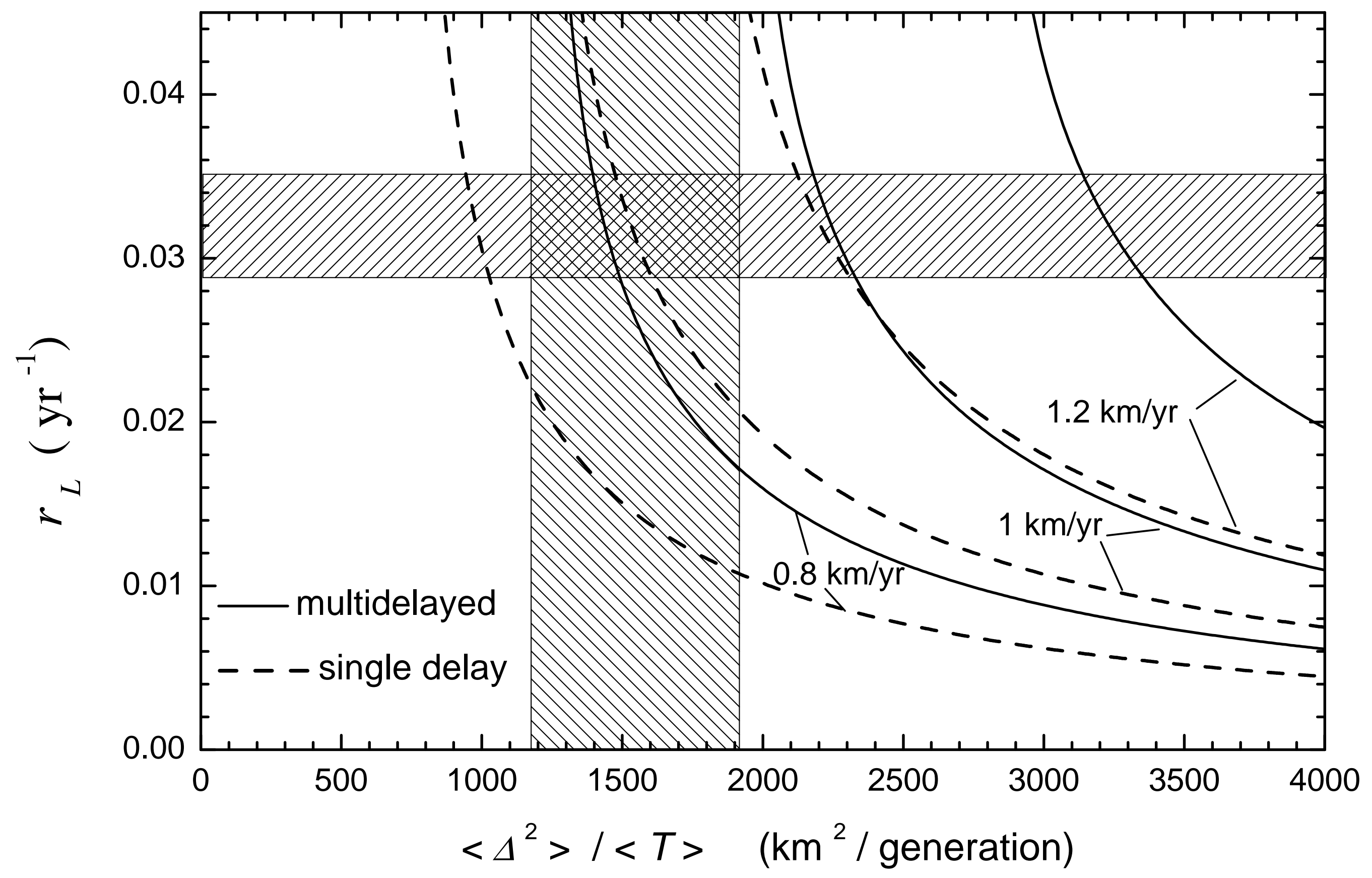


Fort and Pujol, Fig. 12

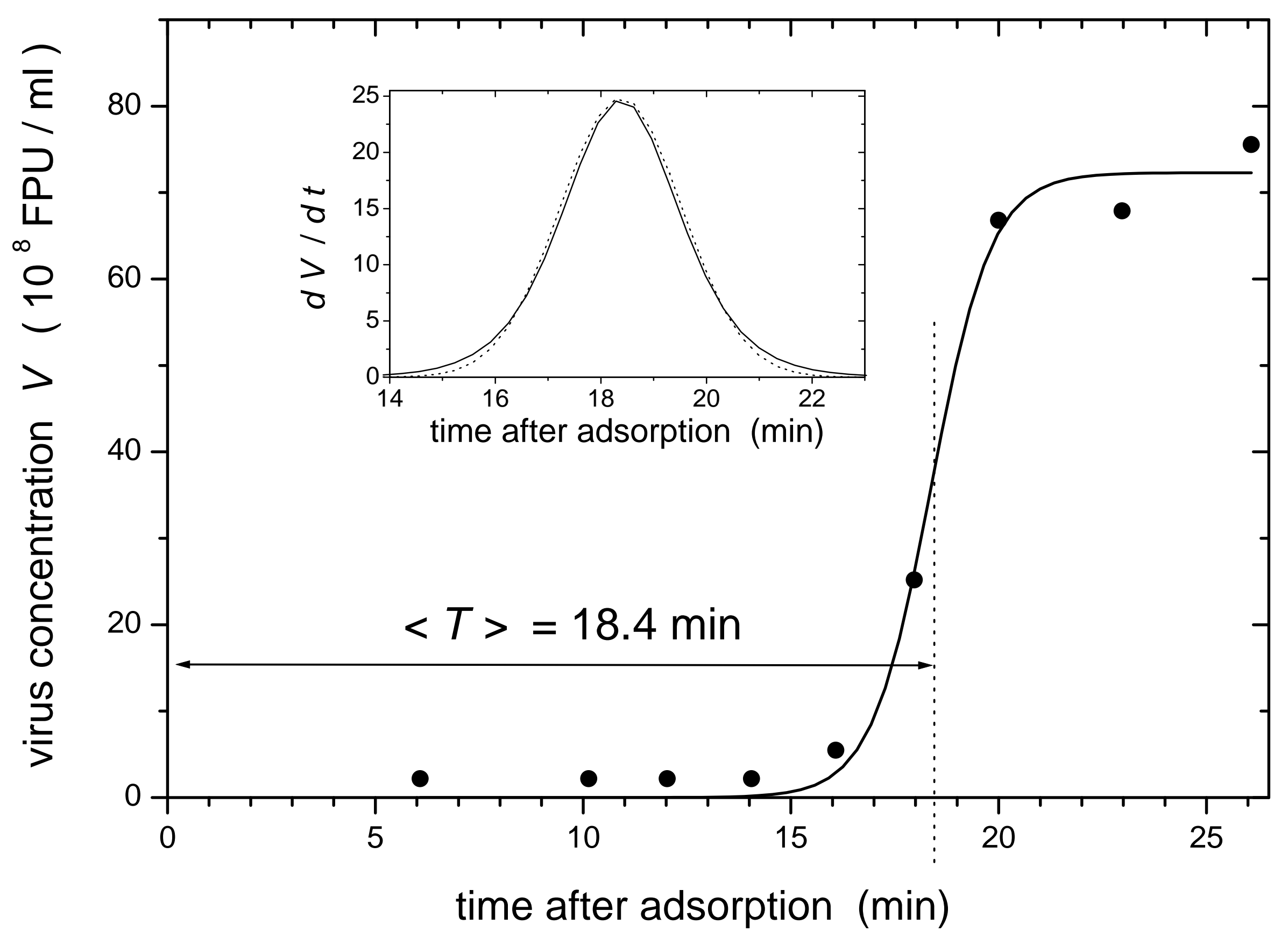


Fort and Pujol, Fig. 13

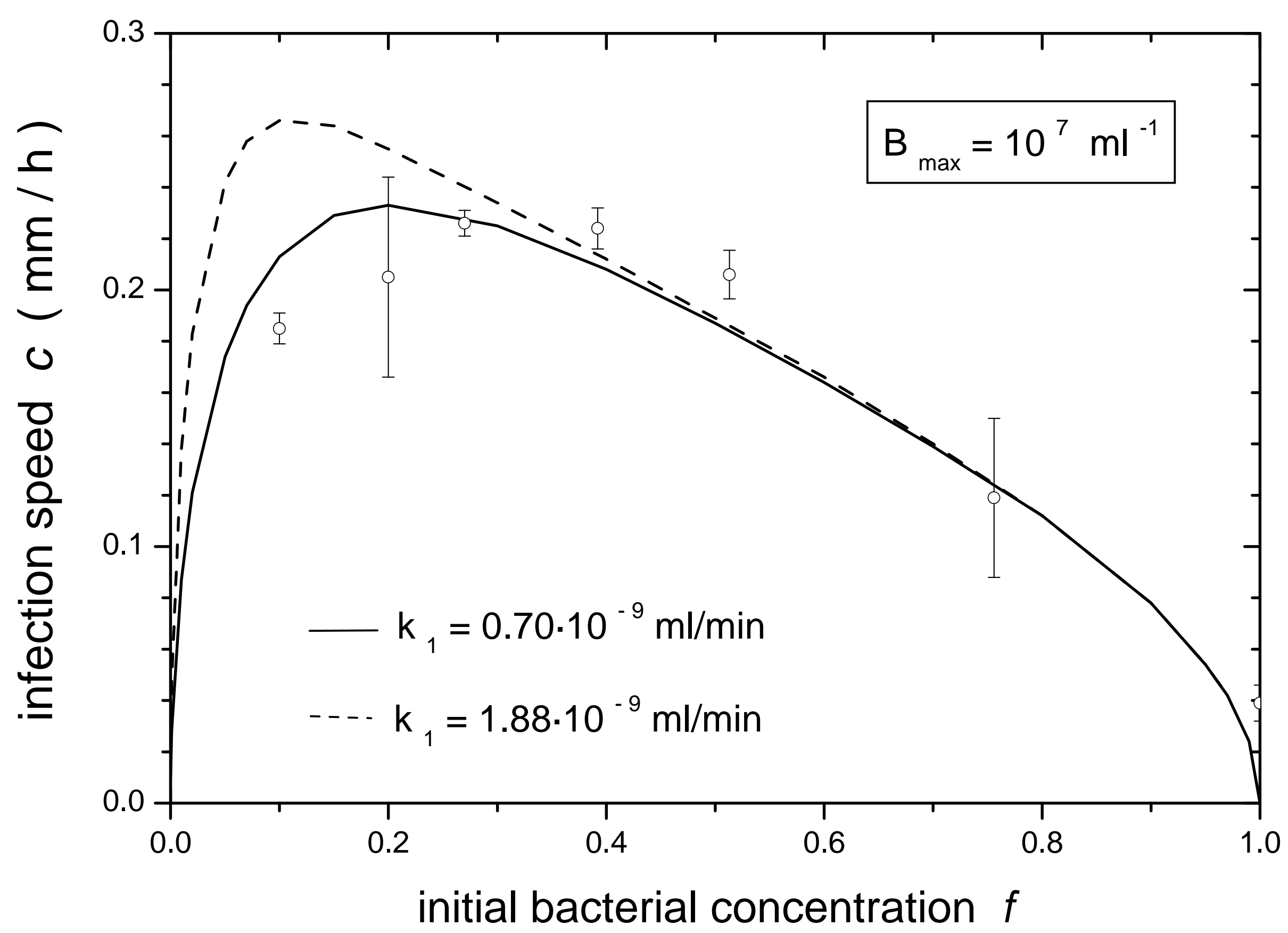


Fort and Pujol, Fig. 14
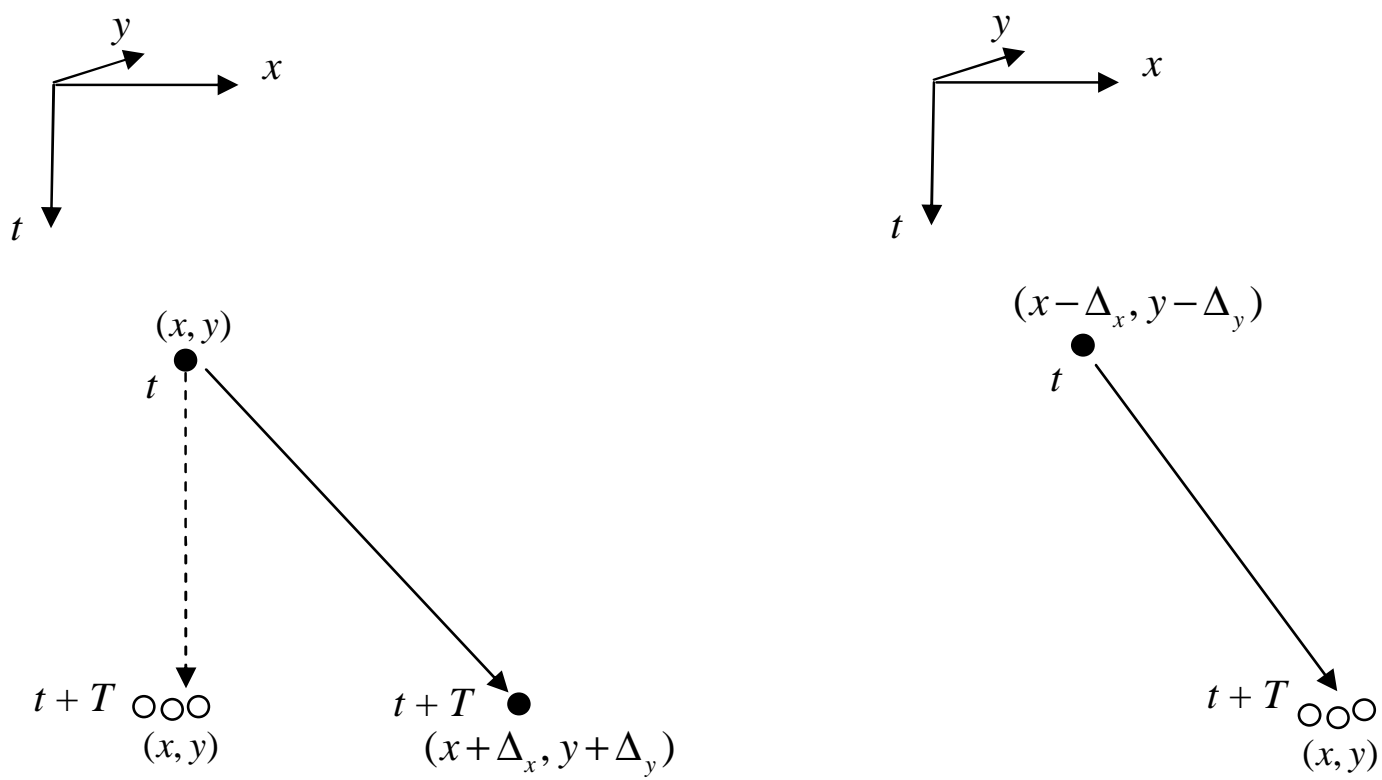

(a)

(b) 
Fort and Pujol, Fig. 15

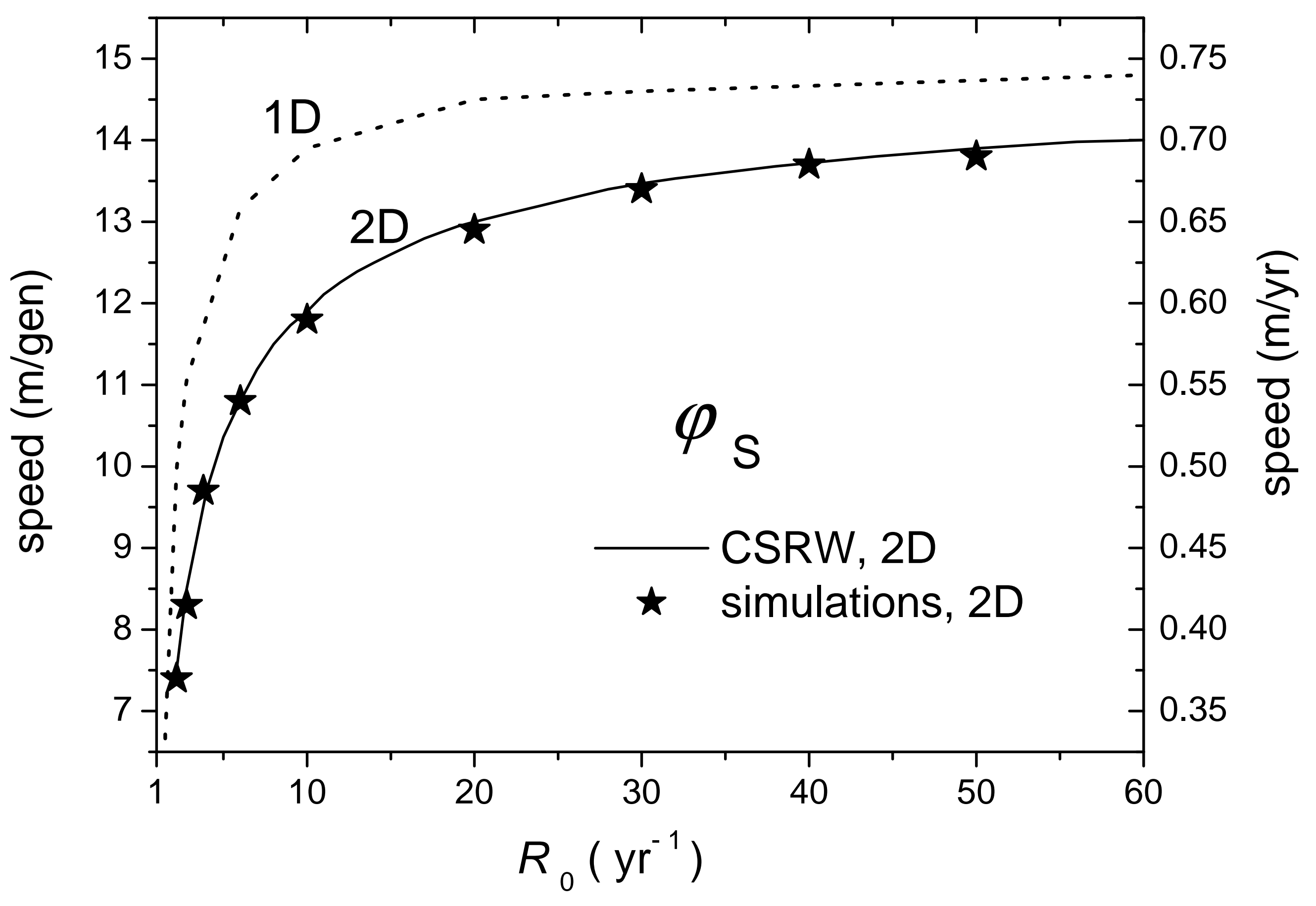


Fort and Pujol, Fig. 16

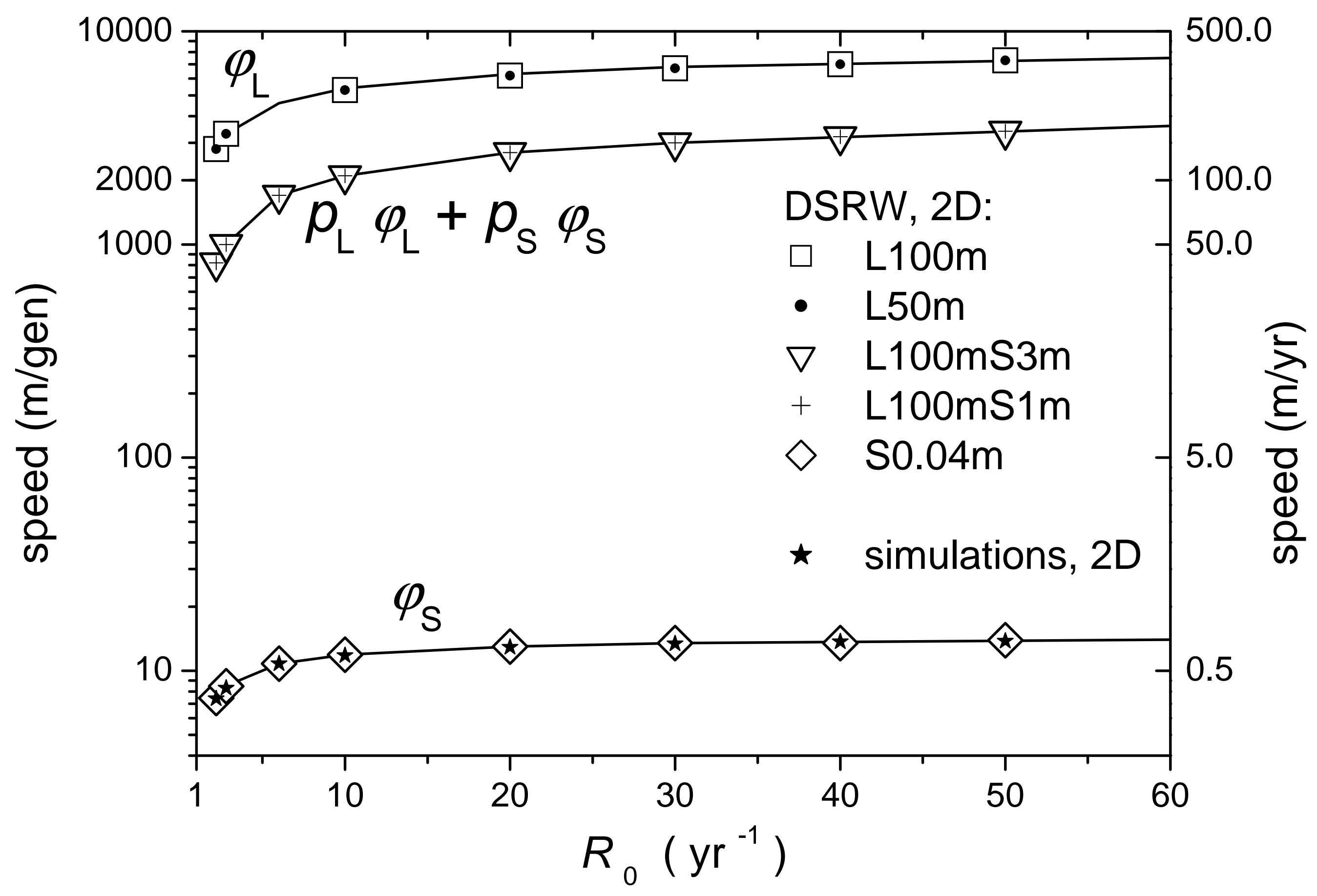


Fort and Pujol, Fig. 17
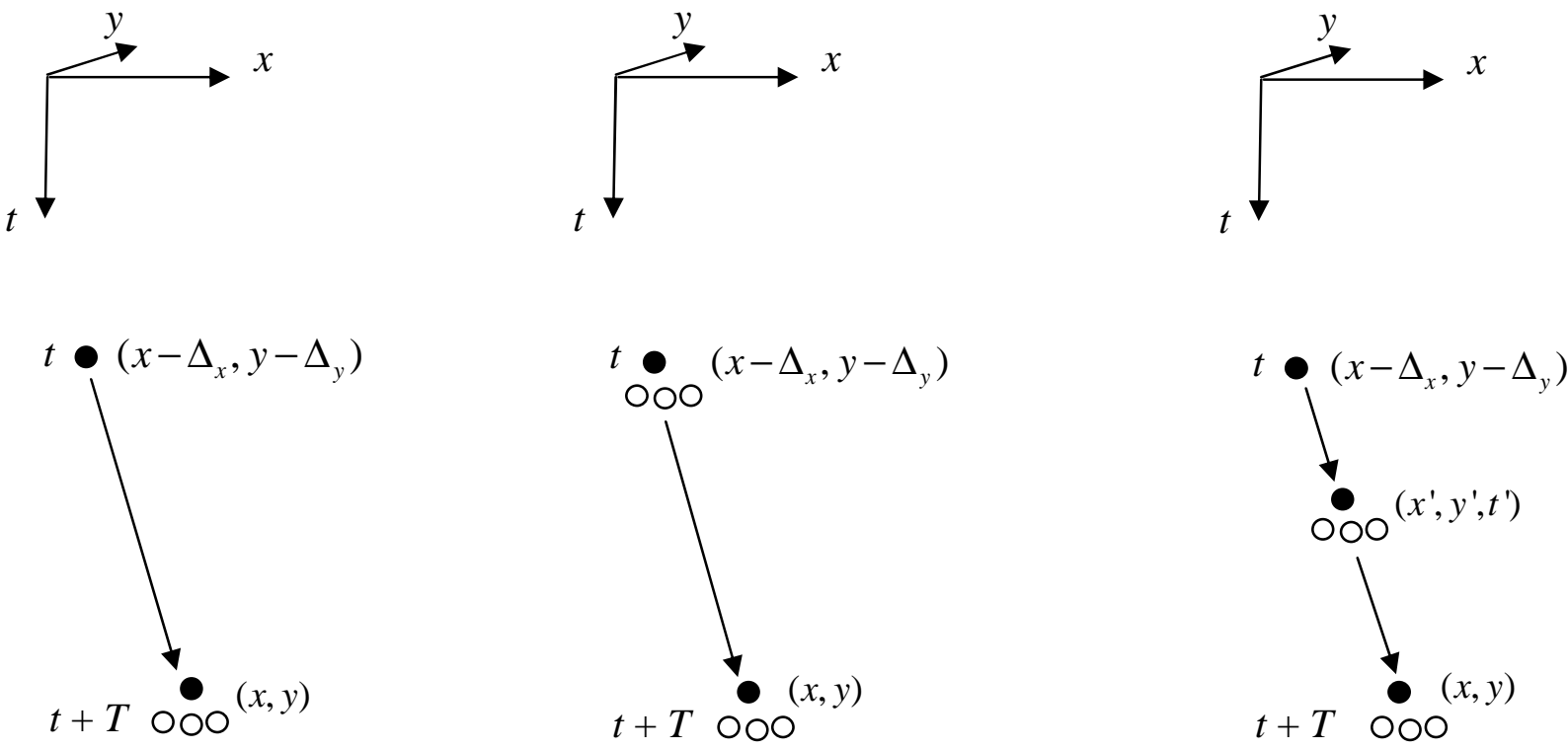

(a)

(b)

(c) 


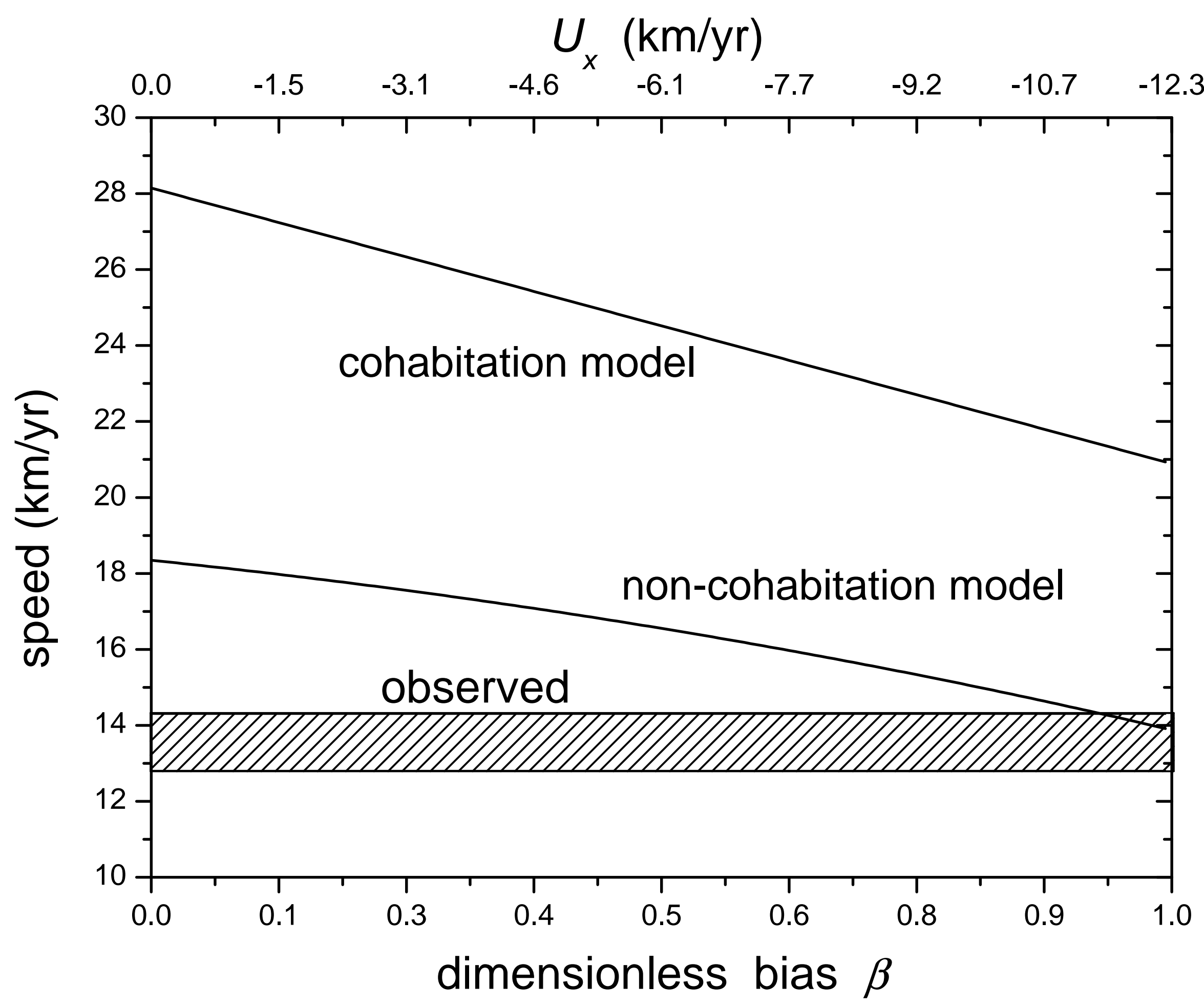

Fort and Pujol Fig.18 
Fort and Pujol, Fig. 19

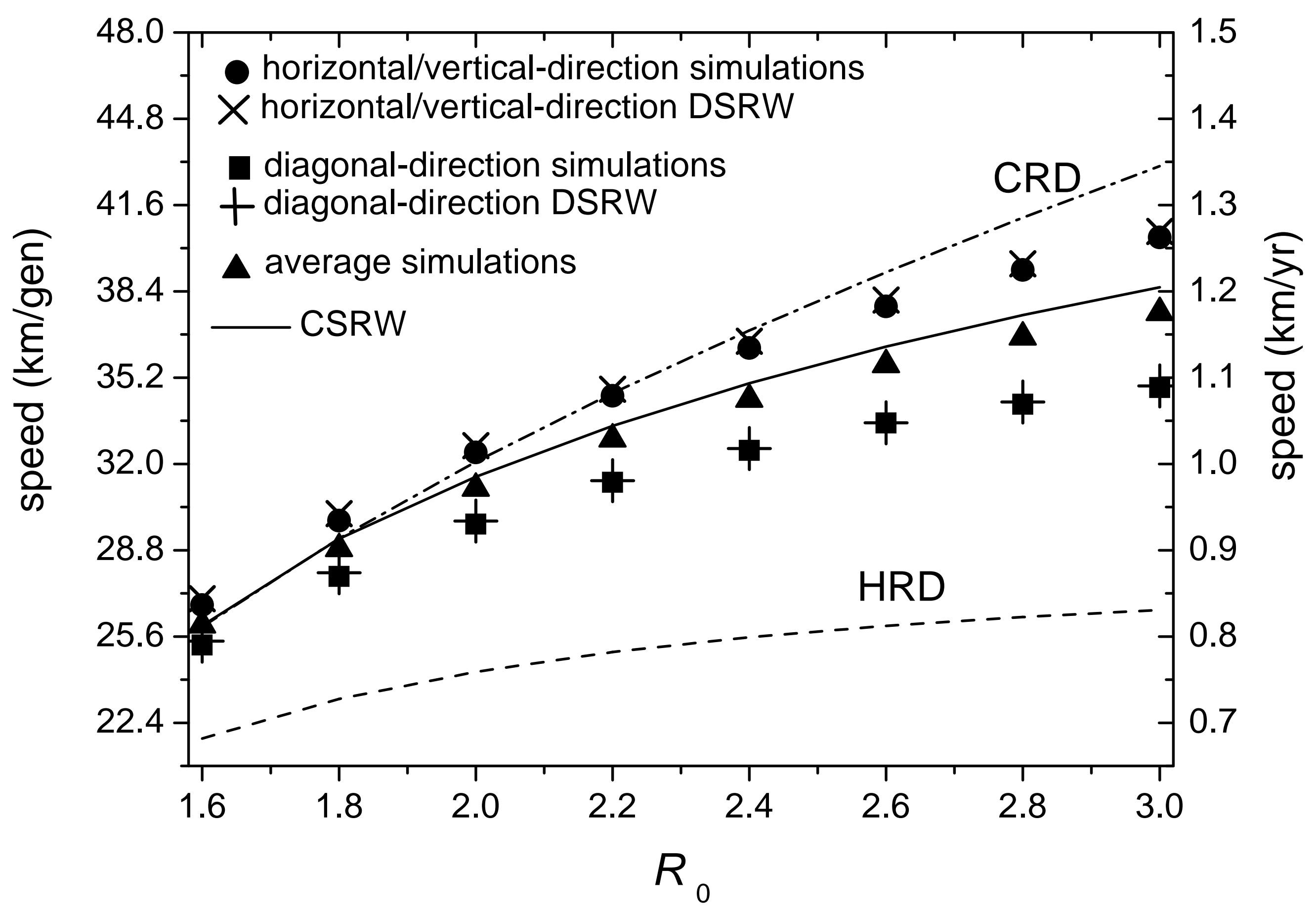


Fort and Pujol, Fig. 20

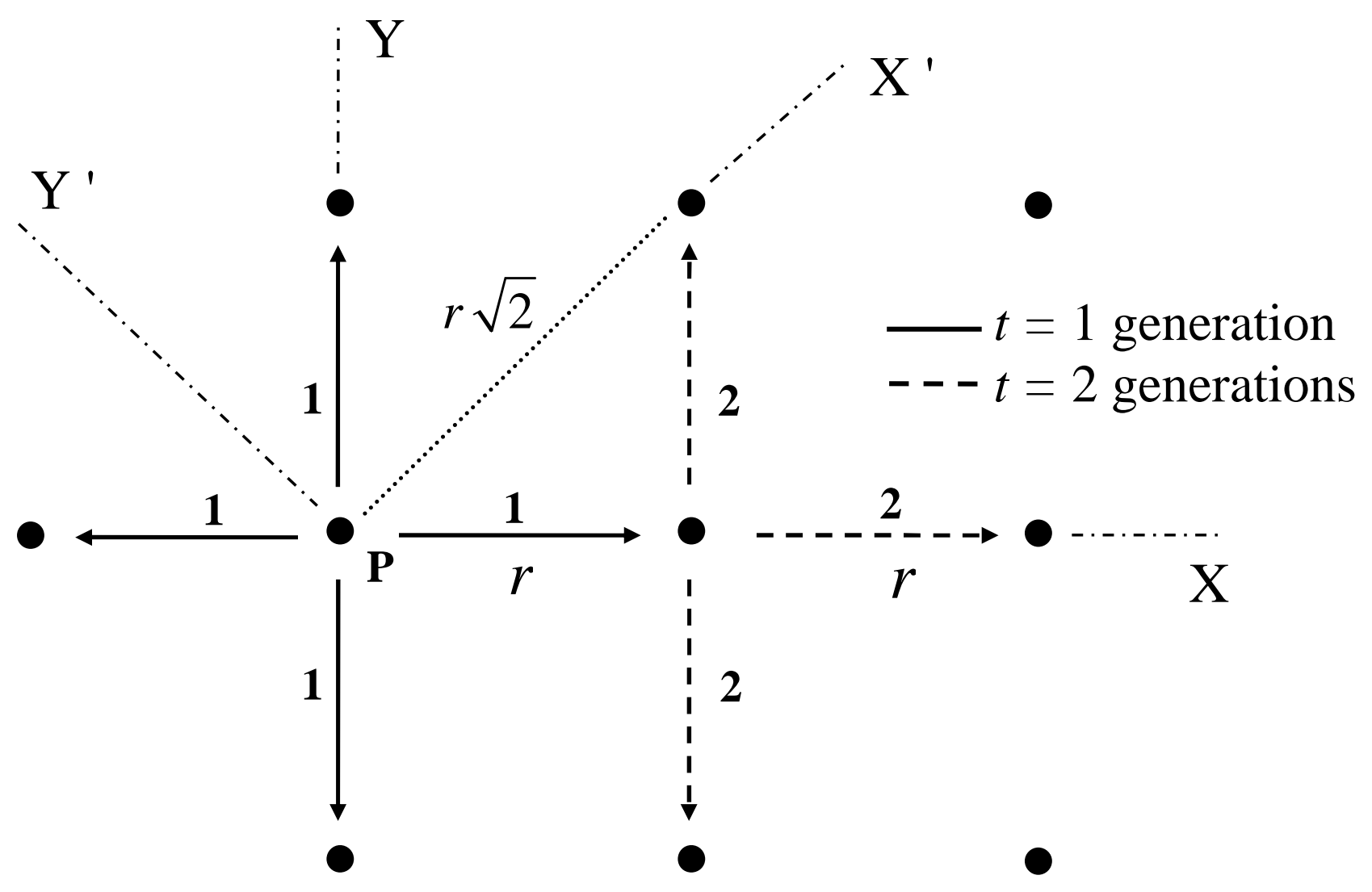


Fort and Pujol, Fig. 21 (a)

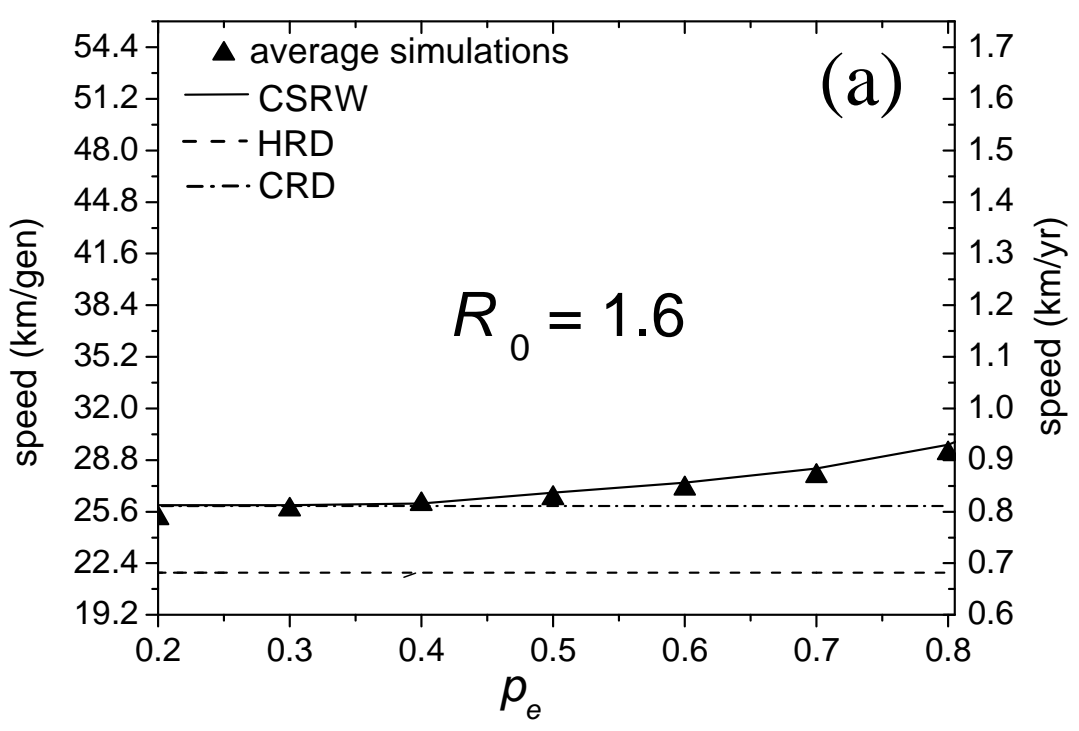

Fort and Pujol, Fig. 21 (b)

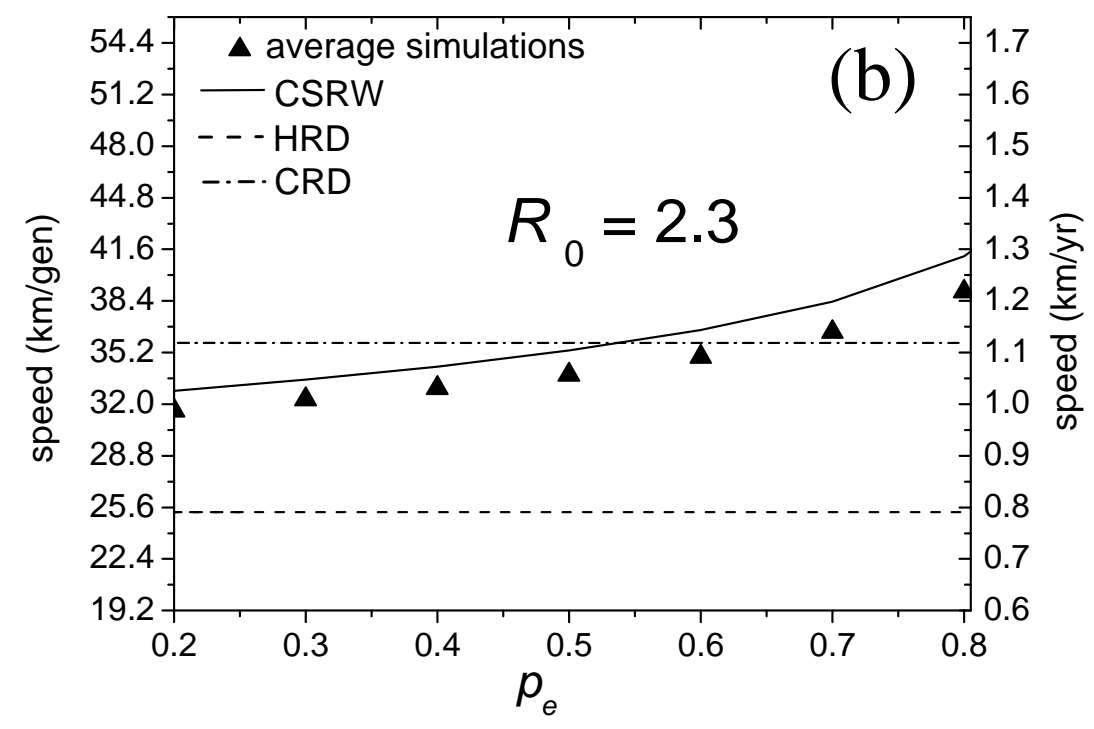

Fort and Pujol, Fig. 21 (c)

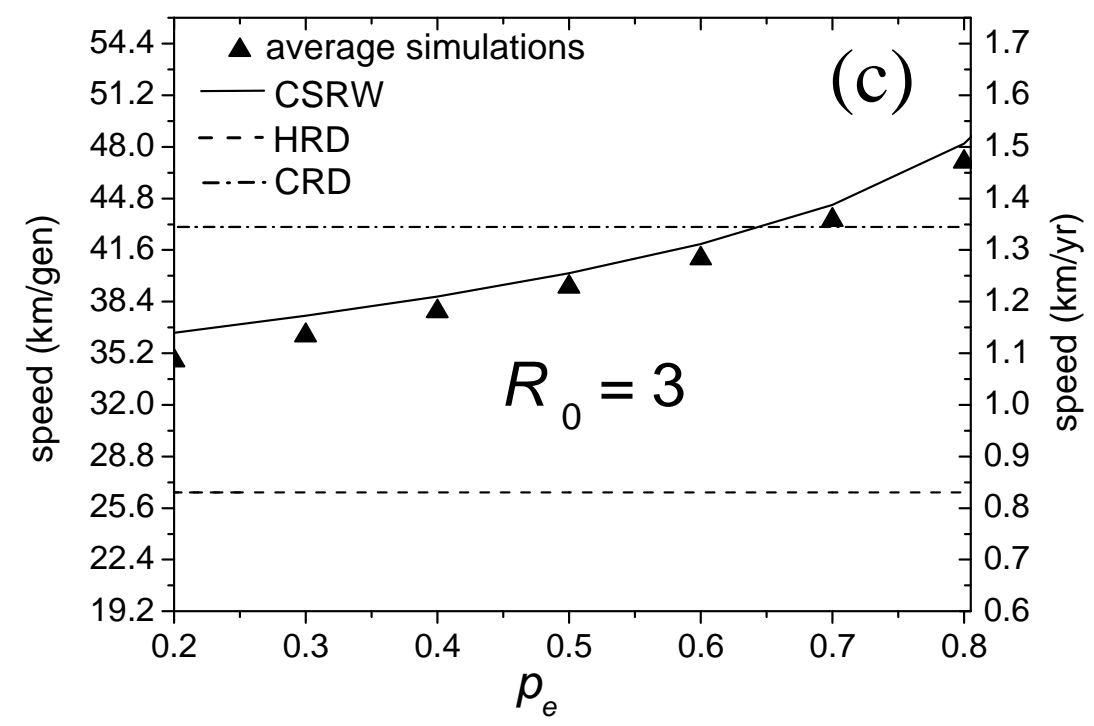


Fort and Pujol, Fig. 22

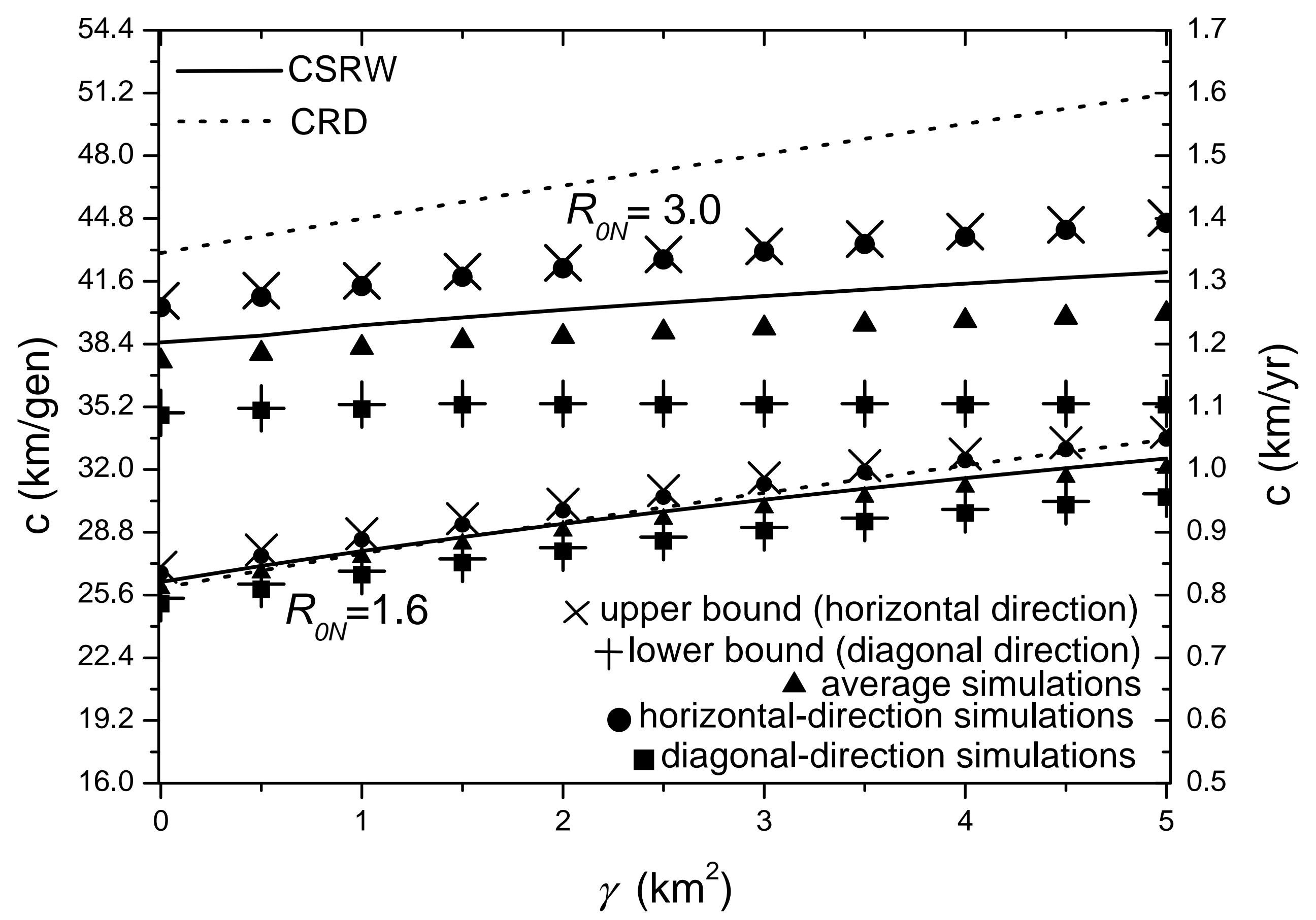

